\title{
All-genus open-closed mirror symmetry for affine toric Calabi-Yau 3-orbifolds
}

\author{
Bohan Fang, Chiu-Chu Melissa Liu and Zhengyu Zong
}

\begin{abstract}
The remodeling conjecture proposed by Bouchard-Klemm-Mariño-Pasquetti relates all-genus open and closed Gromov-Witten invariants of a semi-projective toric CalabiYau 3-manifold/3-orbifold $\mathcal{X}$ to the Eynard-Orantin invariants of the mirror curve of $\mathcal{X}$. In this paper, we present a proof of the remodeling conjecture for open-closed orbifold Gromov-Witten invariants of an arbitrary affine toric Calabi-Yau 3-orbifold relative to a framed Aganagic-Vafa Lagrangian brane. This can be viewed as an allgenus open-closed mirror symmetry for affine toric Calabi-Yau 3-orbifolds.
\end{abstract}

\section{Introduction}

\subsection{Background and motivation}

Mirror symmetry relates the A-model topological string theory on a Calabi-Yau 3 -fold $\mathcal{X}$ to the B-model topological string theory on another Calabi-Yau 3-fold $\check{\mathcal{X}}$, the mirror of $\mathcal{X}$. The genus $g$ free energy of the topological A-model on $\mathcal{X}$ is mathematically defined as a generating function $F_{g}^{\mathcal{X}}$ of genus $g$ Gromov-Witten invariants of $\mathcal{X}$ which is a function on a (formal) neighborhood of the large radius limit in the (complexified) Kähler moduli of $\mathcal{X}$. The genus $g$ free energy of the topological B-model on $\check{\mathcal{X}}$ is a section of $\mathbb{L}^{2-2 g}$, where $\mathbb{L}$ is a line bundle over the complex moduli of $\check{\mathcal{X}}$, so locally it is a function $\check{F}_{g}^{\check{X}}$ on the complex moduli of $\check{\mathcal{X}}$. A mathematical consequence of mirror symmetry is $\check{F}_{g}^{\check{\mathcal{X}}}=F_{g}^{\mathcal{X}}+\delta_{g, 0} a_{0}+\delta_{g, 1} a_{1}$ under the mirror map, where $a_{0}$ (respectively, $a_{1}$ ) is a cubic (respectively, linear) function in Kähler parameters. The mirror map and $\check{F}_{0}^{\check{\mathcal{X}}}$ are determined by period integrals of a holomorphic 3 -form on $\check{\mathcal{X}}$. Period integrals of Calabi-Yau complete intersections in toric manifolds can be expressed in terms of explicit hypergeometric functions.

Let $Q$ be the quintic 3-fold, which is a Calabi-Yau hypersurface in $\mathbb{P}^{4}$, and let $\check{Q}$ be the mirror of $Q$. Candelas, de la Ossa, Green, and Parkes computed $\check{F}_{0}^{\breve{Q}}$ and the mirror map explicitly and obtained a conjectural formula for the number of rational curves of arbitrary degree in $Q$; this formula was first proved independently by Givental [Giv96] and Lian-Liu-Yau [LLY97], who later extended their results to Calabi-Yau complete intersections in projective toric manifolds [Giv98, LLY99a, LLY99b]. Their proofs rely on a good understanding of genus zero GromovWitten theory of $Q$. The mirror formula for $F_{1}^{Q}$ was conjectured by Bershadsky-Cecotti-Ooguri-

Received 4 April 2019, accepted in final form 4 April 2019.

2010 Mathematics Subject Classification 14N35 (primary), 14J33 (secondary).

Keywords: open Gromov-Witten invariants, mirror symmetry, topological recursion.

This journal is (C) Foundation Compositio Mathematica 2020. This article is distributed with Open Access under the terms of the Creative Commons Attribution Non-Commercial License, which permits non-commercial reuse, distribution, and reproduction in any medium, provided that the original work is properly cited. For commercial re-use, please contact the Foundation Compositio Mathematica.

The research of the first author is partially supported by NSF DMS-1206667. The research of the second and third authors is partially supported by NSF DMS-1159416. 
Vafa (BCOV for short) [BCOV93] and first proved by Zinger [Zin09]. Combining the techniques of BCOV, results of Yamaguchi-Yau [YY04], and boundary conditions, Huang-Klemm-Quacken [HKQ09] proposed a mirror conjecture on $F_{g}^{Q}$ up to $g=51$. Maulik-Pandharipande provided a mathematical determination of Gromov-Witten invariants of $Q$ in all genera and degrees [MP06]. In general, it is very difficult to evaluate higher-genus Gromov-Witten invariants of a compact Calabi-Yau 3-fold.

In contrast, higher-genus Gromov-Witten invariants of toric Calabi-Yau 3-folds (which must be non-compact) are much better understood. In general, Gromov-Witten invariants are defined for projective manifolds (or, more generally, compact almost-Kähler manifolds), but in the toric case one may use localization to define Gromov-Witten invariants of certain noncompact toric manifolds. By virtual localization [GP99], all-genus Gromov-Witten invariants of toric manifolds can be reduced to Hodge integrals, which can be evaluated by effective algorithms. When the toric manifold $\mathcal{X}$ is a Calabi-Yau 3-fold, the topological vertex [AKMV05, LLLZ09, MOOP11] provides a much more efficient algorithm for computing Gromov-Witten invariants of $\mathcal{X}$, as well as open Gromov-Witten invariants of $\mathcal{X}$ relative to an Aganagic-Vafa Lagrangian brane $\mathcal{L}$ (defined in [KL01, DF05, Liu02, LLLZ09] in several ways), in all genera and degrees. The topological B-model on the mirror $\check{\mathcal{X}}$ of a smooth semi-projective toric Calabi-Yau 3-fold $\mathcal{X}$ can be reduced to a theory on the mirror curve of $\mathcal{X}$. Under mirror symmetry, $F_{0}^{\mathcal{X}}$ corresponds to integrals of 1 -forms on the mirror curve along loops, whereas the generating function $F_{0,1}^{\mathcal{X}, \mathcal{L}}$ of genus zero open Gromov-Witten invariants (which count holomorphic disks in $\mathcal{X}$ bounded by $\mathcal{L}$ ) corresponds to integrals of 1 -forms on the mirror curve along paths [AV00, AKV02]. On the basis of the work of Eynard-Orantin [EO07] and Mariño [Mar08], Bouchard-Klemm-Mariño-Pasquetti [BKMP09] proposed a new formalism of the topological B-model on $\mathcal{X}$ in terms of the Eynard-Orantin invariants $\omega_{g, n}$ of the mirror curve and conjectured a precise correspondence, known as the remodeling conjecture, between $\omega_{g, n}$ (where $n>0$ ) and the generating function $F_{g, n}^{\mathcal{X}, \mathcal{L}}$ of open Gromov-Witten invariants counting holomorphic maps from bordered Riemann surfaces with $g$ handles and $n$ holes to $\mathcal{X}$ with boundaries in $\mathcal{L}$. This can be viewed as a version of all-genus open mirror symmetry; the closed sector of the remodeling conjecture relates $\omega_{g, 0}$ to $F_{g}^{\mathcal{X}}$. The open string part of the remodeling conjecture for $\mathbb{C}^{3}$ was proved independently by Chen [Che18] and Zhou [Zho09a, Zho09b]. The free energy part of the remodeling conjecture for $\mathbb{C}^{3}$ [BS12] was proved independently by BouchardCatuneanu-Marchal-Sulkowski [BCMS13] and Zhu [Zhu15]. Eynard and Orantin provided a proof of the remodeling conjecture for general smooth semi-projective toric Calabi-Yau 3-folds in $[\mathrm{EO} 15]$.

Bouchard-Klemm-Mariño-Pasquetti have extended the remodeling conjecture to toric Calabi-Yau 3-orbifolds [BKMP10]. Topological string theory on orbifolds was constructed decades ago by physicists [DHVW85, DHVW86], and many works followed in both mathematics and physics (for example, [HV87, DFMS87, BR87, Kni87, Roa90, CV92]). Zaslow discussed orbifold quantum cohomology [Zas93] along with many examples, in both abelian and non-abelian quotients. Later, the mathematical definition of orbifold Gromov-Witten theory and quantum cohomology was laid by Chen-Ruan [CR02] in the symplectic setting and Abramovich-Graber-Vistoli [AGV02, AGV08] in the algebraic setting. Orbifold Gromov-Witten invariants of a toric CalabiYau 3-orbifold $\mathcal{X}$ and open orbifold Gromov-Witten invariants of $\mathcal{X}$ relative to an Aganagic-Vafa Lagrangian brane in $\mathcal{X}$ can be expressed in terms of the orbifold Gromov-Witten vertex, which is a generating function of abelian Hurwitz-Hodge integrals [Ros14]. The algorithm for the topological vertex is equivalent to the Gromov-Witten (GW)/Donaldson-Thomas (DT) correspondence 


\section{B. FAnG, C.-C. M. LiU And Z. Zong}

for smooth toric Calabi-Yau 3-folds [MNOP06]. The GW/DT correspondence has been conjectured for Calabi-Yau 3-orbifolds satisfying the hard Lefshetz condition [BCY12] and proved for toric Calabi-Yau 3-orbifolds with transverse $A_{n}$-singularities [Zon15, RZ13, RZ15, Ros15]. This provides an efficient algorithm for computing closed and open Gromov-Witten invariants of toric Calabi-Yau 3-orbifolds with transverse $A_{n}$-singularities, in all genera and degrees. The precise statement of the GW/DT correspondence for toric Calabi-Yau 3-orbifolds which do not satisfy the hard Lefshetz condition (for example, $\left[\mathbb{C}^{3} / \boldsymbol{\mu}_{3}\right]$, where $\boldsymbol{\mu}_{3}$ acts diagonally) is not known even conjecturally. The remodeling conjecture provides a recursive algorithm to compute closed and open Gromov-Witten invariants of all semi-projective toric Calabi-Yau 3-orbifolds in all genera. Explicit predictions of open orbifold Gromov-Witten invariants of $\left[\mathbb{C}^{3} / \boldsymbol{\mu}_{3}\right]$ are given in $[\mathrm{BKMP} 10]$.

\subsection{Statement of the main result and outline of the proof}

In this paper, we study the remodeling conjecture for all affine toric Calabi-Yau 3-orbifolds $\left[\mathbb{C}^{3} / G\right]$, where $G$ can be any finite subgroup of the maximal torus of $\operatorname{SL}(3, \mathbb{C})$. We consider a general framed Aganagic-Vafa brane $(\mathcal{L}, f)$, where $\mathcal{L} \cong\left[\left(S^{1} \times \mathbb{C}\right) / G\right]$ and $f \in \mathbb{Z}$. We define open-closed orbifold Gromov-Witten invariants of $\mathcal{X}=\left[\mathbb{C}^{3} / G\right]$ relative to $(\mathcal{L}, f)$ as certain equivariant relative orbifold invariants of a toric Calabi-Yau 3-orbifold $\mathcal{Y}_{f}$ relative to a divisor $\mathcal{D}_{f} \cong\left[\mathbb{C}^{2} / \boldsymbol{\mu}_{m}\right]$, where $\boldsymbol{\mu}_{m} \cong \mathbb{Z}_{m}$ is the stabilizer of the $G$-action on $S^{1} \times\{0\} \subset S^{1} \times \mathbb{C}$, and the coarse moduli space $\mathbb{C}^{2} / \boldsymbol{\mu}_{m}$ of $\mathcal{D}_{f}$ is the $A_{m-1}$ surface singularity. We define generating functions of open-closed Gromov-Witten invariants

$$
F_{g, n}^{\mathcal{X},(\mathcal{L}, f)}\left(\tau ; X_{1}, \ldots, X_{n}\right),
$$

which are $H_{\mathrm{CR}}^{*}\left(\mathcal{B} \boldsymbol{\mu}_{m} ; \mathbb{C}\right)^{\otimes n}$-valued formal power series in A-model closed string coordinates $\boldsymbol{\tau}=$ $\left(\tau_{1}, \ldots, \tau_{p}\right)$ and A-model open string coordinates $X_{1}, \ldots, X_{n}$; here $H_{\mathrm{CR}}^{*}\left(\mathcal{B} \boldsymbol{\mu}_{m} ; \mathbb{C}\right) \cong \mathbb{C}^{m}$ is the Chen-Ruan orbifold cohomology of the classifying space $\mathcal{B} \boldsymbol{\mu}_{m}$ of $\boldsymbol{\mu}_{m}$. We use the Eynard-Orantin invariants $\omega_{g, n}$ of the framed mirror curve to define B-model potentials

$$
\check{F}_{g, n}\left(\boldsymbol{\tau} ; X_{1}, \ldots, X_{n}\right),
$$

which are $H_{\mathrm{CR}}^{*}\left(\mathcal{B} \boldsymbol{\mu}_{m} ; \mathbb{C}\right)^{\otimes n}$-valued functions in B-model closed string flat coordinates $\boldsymbol{\tau}=\left(\tau_{1}, \ldots\right.$, $\tau_{p}$ ) and B-model open string coordinates $X_{1}, \ldots, X_{n}$, analytic in an open neighborhood of the origin in $\mathbb{C}^{p} \times \mathbb{C}^{n}$. The mirror map relates the B-model flat coordinates $\left(\tau_{1}, \ldots, \tau_{p}\right)$ to the complex parameters $\left(q_{1}, \ldots, q_{p}\right)$ of the framed mirror curve. Our main result is the following.

Theorem 7.19. For any $g \in \mathbb{Z}_{\geqslant 0}$ and $n \in \mathbb{Z}_{>0}$,

$$
\check{F}_{g, n}\left(\boldsymbol{\tau} ; X_{1}, \ldots, X_{n}\right)=(-1)^{g-1+n} F_{g, n}^{\mathcal{X},(\mathcal{L}, f)}\left(\tau ; X_{1}, \ldots, X_{n}\right) .
$$

This is indeed more general than the original conjecture in [BKMP10], which covers the $m=1$ case, that is, the case when $\mathcal{L}$ is on an effective leg.

We now give an outline of our proof of Theorem 7.19. For simplicity, we consider the stable case $2 g-2+n>0$ in this outline. (The unstable cases $(g, n)=(0,1),(0,2)$ will be treated separately.) The proof consists of three steps:

1. (A-model graph sum) In [FLZ16], we used Tseng's orbifold quantum Riemann-Roch theorem [Tse10] to write down a graph sum formula for the total descendant equivariant Gromov-Witten potential of $\mathcal{X}$. We use this formula and localization to derive a graph 


\section{ALL-GENUS OPEN-CLOSED MIRROR SYMMETRY}

sum formula for the A-model potential $F_{g, n}^{\mathcal{X},(\mathcal{L}, f)}$ :

$$
F_{g, n}^{\mathcal{X},(\mathcal{L}, f)}=\sum_{\vec{\Gamma} \in \boldsymbol{\Gamma}_{g, n}(\mathcal{X})} \frac{w_{A}(\vec{\Gamma})}{|\operatorname{Aut}(\vec{\Gamma})|},
$$

where $\boldsymbol{\Gamma}_{g, n}(\mathcal{X})$ is a certain set of decorated graphs, Aut $(\vec{\Gamma})$ is the automorphism group of the decorated graph $\vec{\Gamma}$, and $w_{A}(\vec{\Gamma})$ is the A-model weight of the decorated graph $\vec{\Gamma}$ defined by $(5.9)$.

2. (B-model graph sum) The Eynard-Orantin invariants $\omega_{g, n}$ can be expressed as sums over labeled graphs [KO10, Eyn11, Eyn14, DOSS14]. We use special geometry to obtain the Taylor series expansion of the graph sum formula in [DOSS14, Theorem 3.7] in B-model closed string flat coordinates $\boldsymbol{\tau}=\left(\tau_{1}, \ldots, \tau_{p}\right)$ at $\boldsymbol{\tau}=0$ and derive a graph sum formula for the B-model potential $\check{F}_{g, n}$ :

$$
\check{F}_{g, n}=\sum_{\vec{\Gamma} \in \boldsymbol{\Gamma}_{g, n}(\mathcal{X})} \frac{w_{B}(\vec{\Gamma})}{|\operatorname{Aut}(\vec{\Gamma})|},
$$

where $w_{B}(\vec{\Gamma})$ is the B-model weight of the decorated graph $\vec{\Gamma}$ defined by (7.14).

3. (Comparison of weights) For each decorated graph $\vec{\Gamma} \in \boldsymbol{\Gamma}_{g, n}(\mathcal{X})$, we prove the following identity relating A-model and B-model weights:

$$
w_{B}(\vec{\Gamma})=(-1)^{g-1+n} w_{A}(\vec{\Gamma}) .
$$

\subsection{Remarks on the BKMP remodeling conjecture in the general case}

In [FLZ19], the authors provide a proof of the BKMP remodeling conjecture for all semiprojective toric Calabi-Yau 3-orbifolds. The proof in [FLZ19] relies on (i) the quantization formula for the total descendant potential of equivariant GW theory of GKM orbifolds [Zon16], (ii) the B-model graph sum formula in [DOSS14, Theorem 3.7], (iii) the genus zero mirror theorem for toric DM stacks [CCIT15, CCK15], and (iv) the genus zero open mirror theorem for semi-projective toric Calabi-Yau 3-orbifolds [FLT12]. Item (i) is used to derive the A-model graph sum formula, whereas items (iii) and (iv) are used to match the A-model and B-model graph sums. In the affine case, the proof in this paper is more direct than the specialization of the proof in [FLZ19] to the affine case: the proof in this paper relies on Tseng's orbifold quantum Riemann-Roch theorem [Tse10] and item (ii), but not on items (i), (iii), (iv). The proof in [FLZ19] also relies on the computation of oscillating integrals on a mirror curve in this paper; we obtain the desired result by integrating in the Landau-Ginzburg model and dimensional reduction (Theorem 7.8 ).

\subsection{Overview of the paper}

In Section 2, we describe affine toric Calabi-Yau 3-orbifolds and their mirror curves. In Section 3, for each affine toric Calabi-Yau 3-orbifold $\mathcal{X}$ and a framed Aganagic-Vafa A-brane $(\mathcal{L}, f)$, we construct a relative Calabi-Yau 3-orbifold $\left(\mathcal{Y}_{f}, \mathcal{D}_{f}\right)$, where $\mathcal{X}=\mathcal{Y}_{f} \backslash \mathcal{D}_{f}$. In Section 4, we describe the Chen-Ruan orbifold cohomology of the classifying space $\mathcal{B} G$ of the finite abelian group $G$ and the $\widetilde{T}$-equivariant Chen-Ruan orbifold cohomology of $\mathcal{X}$ and $\mathcal{Y}_{f}$. In Section 5 , we give the precise definition of the A-model partition functions $F_{g, n}^{\mathcal{X},(\mathcal{L}, f)}$ as generating functions of equivariant relative Gromov-Witten invariants of $\left(\mathcal{Y}_{f}, \mathcal{D}_{f}\right)$ and derive the A-model graph sum formula (Theorem 5.5). In Section 6, we study the geometry and topology of the mirror curve. 


\section{B. FAnG, C.-C. M. LiU AND Z. ZonG}

In particular, we clarify the choice of A-cycles and B-cycles and the definition of the B-model flat coordinates. In Section 7, we give the precise definition of the B-model partition functions $\check{F}_{g, n}$, derive the B-model graph sum formula (Theorem 7.15), and complete the proof of the main result (Theorem 7.19).

\section{Affine toric Calabi-Yau 3-orbifolds and their mirrors}

\subsection{The A-model geometry}

Let $\mathbb{T}=\left(\mathbb{C}^{*}\right)^{3}$ and $N=\operatorname{Hom}\left(\mathbb{C}^{*}, \mathbb{T}\right) \cong \mathbb{Z}^{3}$, and let $M=\operatorname{Hom}\left(\mathbb{T}, \mathbb{C}^{*}\right)=\operatorname{Hom}(N, \mathbb{Z})$. Let $\sigma \subset N_{\mathbb{R}}:=N \otimes_{\mathbb{Z}} \mathbb{R} \cong \mathbb{R}^{3}$ be a simplicial cone spanned by $b_{1}, b_{2}, b_{3} \in N$ such that the simplicial affine toric variety $X_{\sigma}:=\operatorname{Spec} \mathbb{C}\left[\sigma^{\vee} \cap M\right]$ has trivial canonical divisor. Then there exists a $u \in M$ such that $\left\langle u, b_{1}\right\rangle=\left\langle u, b_{2}\right\rangle=\left\langle u, b_{3}\right\rangle=1$. We may choose a $\mathbb{Z}$-basis $\mathrm{u}_{1}, \mathrm{u}_{2}, \mathrm{u}_{3}$ of $M$ such that $\mathrm{u}_{3}=u$. Let $e_{1}, e_{2}, e_{3}$ be the dual $\mathbb{Z}$-basis of $N$. Then

$$
b_{1}=r e_{1}-s e_{2}+e_{3}, \quad b_{2}=m e_{2}+e_{3}, \quad b_{3}=e_{3},
$$

where $r$ and $m$ are positive integers and $s \in\{0,1, \ldots, r-1\}$.

We have a short exact sequence of abelian groups

$$
1 \rightarrow G \rightarrow \widetilde{T}=\left(\mathbb{C}^{*}\right)^{3} \stackrel{\phi}{\rightarrow} \mathbb{T}=\left(\mathbb{C}^{*}\right)^{3} \rightarrow 1,
$$

where $\phi\left(\widetilde{t_{1}}, \widetilde{t_{2}}, \widetilde{t}_{3}\right)=\left(\widetilde{t_{1}^{r}}, \widetilde{t}_{1}^{-s} \widetilde{t}_{2}^{m}, \widetilde{t}_{1} \widetilde{t}_{2} \widetilde{t}_{3}\right)$.

For $i=1,2,3$, let $\chi_{i}: G \rightarrow \mathbb{C}^{*}$ be the projection to the $i$ th factor. Then $\chi_{1}, \chi_{2}, \chi_{3} \in G^{*}=$ $\operatorname{Hom}\left(G, \mathbb{C}^{*}\right)$, and $\chi_{1} \chi_{2} \chi_{3}=1$. Given a positive integer $r$, let $\boldsymbol{\mu}_{r}:=\left\{z \in \mathbb{C}^{*}: z^{r}=1\right\} \cong \mathbb{Z}_{r}$ be the group of $r$ th roots of unity. The image of $\chi_{1}$ is $\boldsymbol{\mu}_{r}$, and the kernel of $\chi_{1}$ is isomorphic to $\boldsymbol{\mu}_{m}$. We have a short exact sequence of finite abelian groups

$$
1 \rightarrow \boldsymbol{\mu}_{m} \rightarrow G \stackrel{\chi_{1}}{\longrightarrow} \boldsymbol{\mu}_{r} \rightarrow 1 \text {. }
$$

Then $X_{\sigma}=\mathbb{C}^{3} / G$, which is the coarse moduli space of $\mathcal{X}:=\left[\mathbb{C}^{3} / G\right]$. Let $N_{\sigma}=\mathbb{Z} b_{1} \oplus \mathbb{Z} b_{2} \oplus \mathbb{Z} b_{3}$. Define

$$
\operatorname{Box}(\sigma)=\left\{v \in N: v=c_{1} b_{1}+c_{2} b_{2}+c_{3} b_{3}, 0 \leqslant c_{i}<1\right\} .
$$

There is a bijection $\operatorname{Box}(\sigma) \rightarrow N / N_{\sigma}$ given by $v \mapsto v+N_{\sigma}$; there is a bijection $\operatorname{Box}(\sigma) \rightarrow G$ given by

$$
c_{1} b_{1}+c_{2} b_{2}+c_{3} b_{3} \mapsto\left(e^{2 \pi \sqrt{-1} c_{1}}, e^{2 \pi \sqrt{-1} c_{2}}, e^{2 \pi \sqrt{-1} c_{3}}\right) .
$$

For example, for $j \in\{1, \ldots, m-1\}$, we have

$$
(0, j, 1)=\frac{j}{m} b_{2}+\left(1-\frac{j}{m}\right) b_{3} \in \operatorname{Box}(\sigma), \quad\left(1, e^{2 \pi \sqrt{-1} j / m}, e^{-2 \pi \sqrt{-1} j / m}\right) \in G .
$$

Define age: $\operatorname{Box}(\sigma) \rightarrow\{0,1,2\}$ by

$$
c_{1} b_{1}+c_{2} b_{2}+c_{3} b_{3} \mapsto c_{1}+c_{2}+c_{3} .
$$

Then we have a disjoint union

$$
\operatorname{Box}(\sigma)=\{0\} \cup\{v \in \operatorname{Box}(\sigma): \operatorname{age}(v)=1\} \cup\{v \in \operatorname{Box}(\sigma): \operatorname{age}(v)=2\} .
$$

Given $h \in G$ and $i \in\{1,2,3\}$, define $c_{i}(h) \in[0,1)$ and age $(h) \in\{0,1,2\}$ by

$$
\begin{aligned}
e^{2 \pi \sqrt{-1} c_{i}(h)} & =\chi_{i}(h), \\
\operatorname{age}(h) & =c_{1}(h)+c_{2}(h)+c_{3}(h) .
\end{aligned}
$$




\section{All-Genus OPEN-ClOSED MIRROR SYMMETRY}

The inverse map $G \rightarrow \operatorname{Box}(\sigma)$ of $(2.1)$ is given by $h \mapsto c_{1}(h) b_{1}+c_{2}(h) b_{2}+c_{3}(h) b_{3}$.

\subsection{The B-model geometry}

There exist $\left(m_{1}, n_{1}\right), \ldots,\left(m_{p}, n_{p}\right) \in \mathbb{Z}^{2}$ such that

$$
\{v \in \operatorname{Box}(\sigma): \operatorname{age}(v)=1\}=\left\{\left(m_{a}, n_{a}, 1\right): a=1, \ldots, p\right\} .
$$

We define $b_{3+a}=\left(m_{a}, n_{a}, 1\right)$ for $a=1, \ldots, p$ and define

$$
H_{f}(X, Y, q)=X^{r} Y^{-s-r f}+Y^{m}+1+\sum_{a=1}^{p} q_{a} X^{m_{a}} Y^{n_{a}-m_{a} f} .
$$

The mirror of $\mathcal{X}$ is a hypersurface in $\mathbb{C}^{2} \times\left(\mathbb{C}^{*}\right)^{2}$ :

$$
\check{\mathcal{X}}_{q}=\left\{(u, v, X, Y) \in \mathbb{C}^{2} \times\left(\mathbb{C}^{*}\right)^{2}: H_{f}(X, Y, q)-u v=0\right\} .
$$

This is a family of non-compact Calabi-Yau 3 -folds parametrized by $q=\left(q_{1}, \ldots, q_{p}\right)$. Different framings $f \in \mathbb{Z}$ give isomorphic $\check{\mathcal{X}}_{q}$ but different superpotentials $X: \check{\mathcal{X}}_{q} \rightarrow \mathbb{C}^{*}$. The framed mirror curve is

$$
\Sigma_{q}=\left\{(X, Y) \in\left(\mathbb{C}^{*}\right)^{2}: H_{f}(X, Y, q)=0\right\}
$$

Then

(i) the hypersurface $\check{\mathcal{X}}_{q}$ is smooth if and only if $\Sigma_{q}$ is smooth;

(ii) all the branch points of $X: \Sigma_{q} \rightarrow \mathbb{C}^{*}$ are simple if and only if the critical points of $X: \check{\mathcal{X}}_{q} \rightarrow$ $\mathbb{C}^{*}$ are isolated and non-degenerate.

Note that $\Sigma_{0}=\left\{(X, Y) \in\left(\mathbb{C}^{*}\right)^{2}: X^{r} Y^{-s-r f}+Y^{m}+1=0\right\}$ is smooth and all the branch points of $X: \Sigma_{0} \rightarrow \mathbb{C}^{*}$ are simple. So properties (i) and (ii) hold for small enough $q$.

Remark 2.1. When $m=1, G=\boldsymbol{\mu}_{r}$, and $q_{a}=0$, the framed mirror curve is given by $X^{r} Y^{-s-r f}+$ $Y+1=0$ or, equivalently,

$$
Y^{s+r f}(1+Y)+X^{r}=0 .
$$

Up to the sign, which is a matter of convention, (2.4) agrees with the following framed mirror curve given by equation (3.28) of [BHLM14]:

$$
Y^{s+r f}(1-Y)-X^{r}=0 .
$$

In this paper, we assume that the framing $f$ is a positive integer.

We define

$$
w_{1}=\frac{1}{r}, \quad w_{2}=\frac{s+r f}{r m}, \quad w_{3}=-w_{1}-w_{2}=\frac{-s-r f-m}{r m} .
$$

For later convenience, we fix two explicit bijections (which are not necessarily group homomorphisms) $\iota: \mathbb{Z}_{r} \times \mathbb{Z}_{m}$ and $\iota^{*}: G^{*} \rightarrow \mathbb{Z}_{r} \times \mathbb{Z}_{m}$ :

- $\iota: \mathbb{Z}_{r} \times \mathbb{Z}_{m} \rightarrow G,(j, \ell) \mapsto \eta_{1}^{j} \eta_{2}^{\ell}$, where

$$
\eta_{1}=\left(e^{2 \pi \sqrt{-1} w_{1}}, e^{2 \pi \sqrt{-1} w_{2}}, e^{2 \pi \sqrt{-1} w_{3}}\right), \quad \eta_{2}=\left(1, e^{2 \pi \sqrt{-1} / m}, e^{-2 \pi \sqrt{-1} / m}\right) .
$$

- $\iota^{*}: \mathbb{Z}_{r} \times \mathbb{Z}_{m} \rightarrow G^{*},(j, \ell) \mapsto \chi_{1}^{j} \chi_{2}^{\ell}$, where $\chi_{1}, \chi_{2} \in G^{*}$ are defined as in Section 2.1. 


\section{The 1-leg framed orbifold topological vertex}

Let $L=\left\{\left(z_{1}, z_{2}, z_{3}\right) \in \mathbb{C}^{3}:\left|z_{1}\right|^{2}-\left|z_{2}\right|^{2}=c,\left|z_{1}\right|^{2}-\left|z_{3}\right|^{2}=c, \Im\left(z_{1} z_{2} z_{3}\right)=0\right\}$, where $c>0$ and $\Im(z)$ means the imaginary part of $z$. Then $L$ is a special Lagrangian of $\mathbb{C}^{3}$ (Harvey-Lawson [HL82]), and $\mathcal{L}:=[L / G]$ is a special Lagrangian sub-orbifold of the affine toric Calabi-Yau 3-orbifold $\mathcal{X}=\left[\mathbb{C}^{3} / G\right]$. A framed Aganagic-Vafa A-brane of $\mathcal{X}$ is a pair $(\mathcal{L}, f)$, where $\mathcal{L} \subset \mathcal{X}$ is as above and $f \in \mathbb{Z}$. The 1-leg framed orbifold topological vertex is a relative toric Calabi-Yau 3-orbifold $\left(\mathcal{Y}_{f}, \mathcal{D}_{f}\right)$ associated with $(\mathcal{X}, \mathcal{L}, f)$.

\subsection{The toric graph}

The lattice $N_{\sigma}=\mathbb{Z} b_{1} \oplus \mathbb{Z} b_{2} \oplus \mathbb{Z} b_{3}$ is a sublattice of $N=\mathbb{Z} e_{1} \oplus \mathbb{Z} e_{2} \oplus \mathbb{Z} e_{3}$ of index $r m$, and $\left\{b_{1}, b_{2}, b_{3}\right\}$ is a $\mathbb{Q}$-basis of $N_{\mathbb{Q}}:=N \otimes_{\mathbb{Z}} \mathbb{Q}$. Let $\left\{\mathrm{w}_{1}, \mathrm{w}_{2}, \mathrm{w}_{3}\right\}$ be the dual $\mathbb{Q}$-basis of $M_{\mathbb{Q}}:=M \otimes_{\mathbb{Z}} \mathbb{Q}$. Then

Note that

$$
\mathrm{w}_{1}=\frac{1}{r} \mathrm{u}_{1}, \quad \mathrm{w}_{2}=\frac{s}{r m} \mathrm{u}_{1}+\frac{1}{m} \mathrm{u}_{2}, \quad \mathrm{w}_{3}=-\frac{s+m}{r m} \mathrm{u}_{1}-\frac{1}{m} \mathrm{u}_{2}+\mathrm{u}_{3} .
$$

$$
\mathrm{w}_{1}+\mathrm{w}_{2}+\mathrm{w}_{3}=\mathrm{u}_{3}
$$

is the weight of the $\mathbb{T}$-action on $T_{\mathfrak{p}} \mathcal{X}$, where $\mathfrak{p}=\mathcal{B} G$ is the unique $\mathbb{T}$-fixed point in the toric 3 -orbifold $\mathcal{X}$. Let $\mathbb{T}^{\prime}$ be the kernel of $\mathrm{u}_{3} \in M=\operatorname{Hom}\left(\mathbb{T}, \mathbb{C}^{*}\right)$.

The relative formal toric Calabi-Yau (FTCY) graph of the 1-leg framed orbifold topological vertex is shown in Figure 1. This generalizes the 1-leg framed topological vertex [LLLZ09].

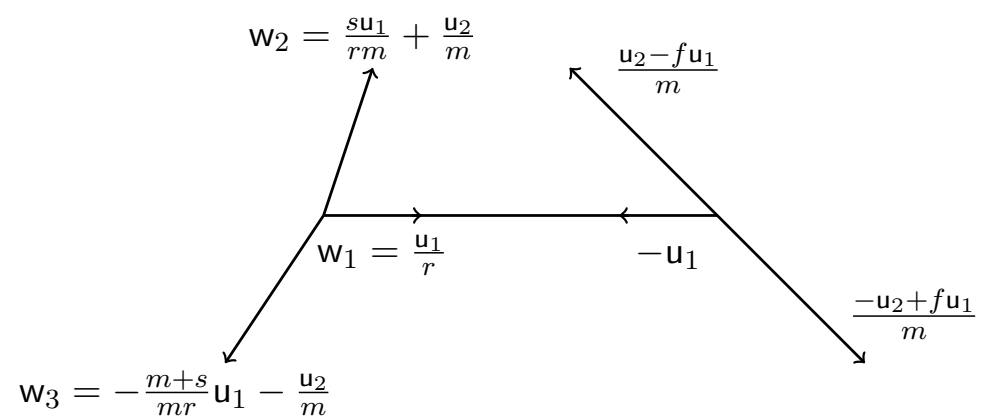

FiguRE 1. The toric graph of the 1-leg framed orbifold topological vertex

\subsection{The fan}

The toric graph in Figure 1 defines a relative toric Calabi-Yau 3-orbifold $\mathcal{Y}_{f}$ which is a partial compactification of $\mathcal{X}$. We now describe the fan $\Sigma_{f}$ defining the coarse moduli space $Y_{f}$ of $\mathcal{Y}_{f}$. Let

$$
v_{1}=b_{1}=r e_{1}-s e_{2}+e_{3}, \quad v_{2}=b_{2}=m e_{2}+e_{3}, \quad v_{3}=b_{3}=e_{3}, \quad v_{4}=-e_{1}-f e_{2} .
$$

Let $\sigma$ be the 3 -cone spanned by $v_{1}, v_{2}, v_{3}$, as before; let $\sigma^{\prime}$ be the 3 -cone spanned by $v_{2}, v_{3}, v_{4}$. Let $\tau$ be the 2-cone $\sigma \cap \sigma^{\prime}$, and let $\rho_{i}$ be the 1 -cone spanned by $v_{i}$. Then

$$
\Sigma_{f}(1)=\left\{\rho_{1}, \rho_{2}, \rho_{3}, \rho_{4}\right\}, \quad \Sigma_{f}(3)=\left\{\sigma, \sigma^{\prime}\right\} .
$$

The coarse moduli space $D_{f}$ is the $\mathbb{T}$-invariant divisor in $Y_{f}$ associated with $\rho_{4}$. The pair $\left(\mathcal{Y}_{f}, \mathcal{D}_{f}\right)$ is a relative toric Calabi-Yau 3-orbifold, where the relative Calabi-Yau condition is $K_{\mathcal{Y}_{f}}+\mathcal{D}_{f}=0$. 


\section{AlL-GENUS OPEN-CLOSED MIRROR SYMMETRY}

Let $\mathcal{D}_{i}$ be the $\mathbb{T}$-invariant divisor associated with $\rho_{i}$. Then

$$
K_{\mathcal{Y}_{f}}=-\mathcal{D}_{1}-\mathcal{D}_{2}-\mathcal{D}_{3}-\mathcal{D}_{4}, \quad K_{\mathcal{Y}_{f}}+\mathcal{D}_{f}=-\mathcal{D}_{1}-\mathcal{D}_{2}-\mathcal{D}_{3} .
$$

Note that $K_{\mathcal{X}_{f}}+\mathcal{D}_{f}$ is the principal $\mathbb{T}$-divisor associated with $\chi^{\mathrm{u}_{3}} \in \mathbb{C}[M]$.

\subsection{The root construction}

In this subsection, we give another description of the relative toric 3 -orbifold $\left(\mathcal{Y}_{f}, \mathcal{D}_{f}\right)$. Let $\mathfrak{p}_{0}=[0,1]$ and $\mathfrak{p}_{\infty}=[1,0]$ be the two torus-fixed points in $\mathbb{P}(1, r)$, where $\mathbb{P}(1, r)$ is the weighted projective line with an orbifold point of order $r$. Then $\mathfrak{p}_{0}$ is the unique stacky point in $\mathbb{P}(1, r)$, and any $\mathbb{C}^{*}$-equivariant line bundle on $\mathbb{P}(1, r)$ is of the form

$$
\mathcal{O}_{\mathbb{P}(1, r)}\left(a \mathfrak{p}_{0}+b \mathfrak{p}_{\infty}\right),
$$

where $a, b \in \mathbb{Z}$. We have

$$
\operatorname{deg} \mathcal{O}_{\mathbb{P}(1, r)}\left(a \mathfrak{p}_{0}+b \mathfrak{p}_{\infty}\right)=\frac{a}{r}+b .
$$

Let $p: \mathfrak{l} \rightarrow \mathbb{P}(1, r)$ be the $\mathbb{Z}_{m}$-gerbe obtained by applying the $m$ th root construction to the equivariant line bundle $\mathcal{O}_{\mathbb{P}(1, r)}\left(s \mathfrak{p}_{0}+f \mathfrak{p}_{\infty}\right)$. Let $\mathcal{L}_{2}$ be the tautological line bundle over $\mathfrak{l}$, so that

$$
\mathcal{L}_{2}^{\otimes m}=p^{*} \mathcal{O}_{\mathbb{P}(1, r)}\left(s \mathfrak{p}_{0}+f \mathfrak{p}_{\infty}\right) .
$$

Then $\operatorname{deg} \mathcal{L}_{2}=s / r m+f / m=w_{2}$. Let

$$
\mathcal{L}_{3}:=\mathcal{L}_{2}^{-1} \otimes p^{*} \mathcal{O}_{\mathbb{P}(1, r)}\left(-\mathfrak{p}_{0}\right) .
$$

Then $\operatorname{deg} \mathcal{L}_{3}=-s / r m-f / m-1 / r=w_{3}$. The toric 3-orbifold $\mathcal{Y}_{f}$ is the total space of the rank 2 vector bundle

$$
V_{f}:=\mathcal{L}_{2} \oplus \mathcal{L}_{3} \rightarrow \mathfrak{l}
$$

The divisor $\mathcal{D}_{f}$ is the fiber of $V_{f}$ over the stacky point $p^{-1}\left(\mathfrak{p}_{\infty}\right)$ in $\mathfrak{l}$.

When $G$ is trivial, we have $r=m=1, s=0, \mathfrak{l}=\mathbb{P}(1, r)=\mathbb{P}^{1}$, and $V_{f}=\mathcal{O}_{\mathbb{P}^{1}}(f) \oplus \mathcal{O}_{\mathbb{P}^{1}}(-f-1)$ $\rightarrow \mathbb{P}^{1}$.

\section{Chen-Ruan orbifold cohomology}

In this section, we describe the Chen-Ruan orbifold cohomology [CR04] of the classifying space $\mathcal{B} G$ of the finite abelian group $G$ and the $\widetilde{T}$-equivariant Chen-Ruan orbifold cohomology of $\mathcal{X}=\left[\mathbb{C}^{3} / G\right]$ and $\mathcal{Y}_{f}$. The Chen-Ruan orbifold cohomology of the classifying space of any finite group is described in [JK02].

\subsection{Chen-Ruan orbifold cohomology of $\mathcal{B G}$}

The inertia stack of $\mathcal{B} G$ is

$$
\mathcal{I} \mathcal{B} G=\bigcup_{h \in G}(\mathcal{B} G)_{h}, \quad \text { where } \quad(\mathcal{B} G)_{h}=[\{h\} / G] \cong \mathcal{B} G .
$$

As a graded vector space over $\mathbb{C}$,

$$
H_{\mathrm{CR}}^{*}(\mathcal{B} G ; \mathbb{C})=H^{*}(\mathcal{I} \mathcal{B} G ; \mathbb{C})=\bigoplus_{h \in G} H^{0}\left((\mathcal{B} G)_{h} ; \mathbb{C}\right),
$$


B. FAnG, C.-C. M. LiU And Z. Zong

where $H^{0}\left((\mathcal{B} G)_{h} ; \mathbb{C}\right)=\mathbb{C} \mathbf{1}_{h}$. The orbifold Poincaré pairing of $H_{\mathrm{CR}}^{*}(\mathcal{B} G ; \mathbb{C})$ is given by

$$
\left\langle\mathbf{1}_{h}, \mathbf{1}_{h^{\prime}}\right\rangle=\frac{\delta_{h^{-1}, h^{\prime}}}{|G|} .
$$

The orbifold cup product of $H_{\mathrm{CR}}^{*}(\mathcal{B} G ; \mathbb{C})$ is given by $\mathbf{1}_{h} \star \mathbf{1}_{h^{\prime}}=\mathbf{1}_{h h^{\prime}}$.

We now define a canonical basis for the semi-simple algebra $H_{\mathrm{CR}}^{*}(\mathcal{B} G ; \mathbb{C})$. Given a character $\gamma \in G^{*}=\operatorname{Hom}\left(G, \mathbb{C}^{*}\right)$, define

$$
\phi_{\gamma}:=\frac{1}{|G|} \sum_{h \in G} \chi_{\gamma}\left(h^{-1}\right) \mathbf{1}_{h}
$$

Then

$$
H_{\mathrm{CR}}^{*}(\mathcal{B} G ; \mathbb{C})=\bigoplus_{h \in G} \mathbb{C} \mathbf{1}_{h}=\bigoplus_{\gamma \in G^{*}} \mathbb{C} \phi_{\gamma} .
$$

Recall that we have the orthogonality of characters:

(1) For any $\gamma, \gamma^{\prime} \in G^{*}$, we have $\frac{1}{|G|} \sum_{h \in G} \chi_{\gamma}\left(h^{-1}\right) \chi_{\gamma^{\prime}}(h)=\delta_{\gamma, \gamma^{\prime}}$,

(2) For any $h, h^{\prime} \in G$, we have $\frac{1}{|G|} \sum_{\gamma \in G^{*}} \chi_{\gamma}\left(h^{-1}\right) \chi_{\gamma}\left(h^{\prime}\right)=\delta_{h, h^{\prime}}$.

Therefore, $\left\langle\phi_{\gamma}, \phi_{\gamma^{\prime}}\right\rangle=\delta_{\gamma, \gamma^{\prime}} /|G|^{2}$ and $\phi_{\gamma} \star \phi_{\gamma^{\prime}}=\delta_{\gamma, \gamma^{\prime}} \phi_{\gamma}$. Then $\left\{\phi_{\gamma}: \gamma \in G^{*}\right\}$ is a canonical basis of $H_{\mathrm{CR}}^{*}(\mathcal{B} G ; \mathbb{C})$.

\subsection{Equivariant Chen-Ruan orbifold cohomology of $\mathcal{X}=\left[\mathbb{C}^{3} / G\right]$}

Given any $h \in G$, define $c_{i}(h) \in[0,1) \cap \mathbb{Q}$ and age $(h) \in\{0,1,2\}$ by equations (2.2) and (2.3) in Section 2.2 , respectively. Let $\left(\mathbb{C}^{3}\right)^{h}$ denote the $h$-invariant subspace of $\mathbb{C}^{3}$. Then

$$
\operatorname{dim}_{\mathbb{C}}\left(\mathbb{C}^{3}\right)^{h}=\sum_{i=1}^{3} \delta_{c_{i}(h), 0}
$$

The inertial stack of $\mathcal{X}$ is

$$
\mathcal{I X}=\bigcup_{h \in G} \mathcal{X}_{h}, \quad \text { where } \quad \mathcal{X}_{h}=\left[\left(\mathbb{C}^{3}\right)^{h} / G\right]
$$

In particular, $\mathcal{X}_{1}=\left[\mathbb{C}^{3} / G\right]=\mathcal{X}$. As a graded vector space over $\mathbb{C}$,

$$
H_{\mathrm{CR}}^{*}(\mathcal{X} ; \mathbb{C})=\bigoplus_{h \in G} H^{*}\left(\mathcal{X}_{h} ; \mathbb{C}\right)[2 \operatorname{age}(h)]=\bigoplus_{h \in G} \mathbb{C} \mathbf{1}_{h},
$$

where $\operatorname{deg}\left(\mathbf{1}_{h}\right)=2 \operatorname{age}(h) \in\{0,2,4\}$. The orbifold Poincaré pairing of the (non-equivariant) Chen-Ruan orbifold cohomology $H_{\mathrm{CR}}^{*}(\mathcal{X} ; \mathbb{C})$ is given by

$$
\left\langle\mathbf{1}_{h}, \mathbf{1}_{h^{\prime}}\right\rangle_{\mathcal{X}}=\frac{1}{|G|} \delta_{h^{-1}, h^{\prime}} \cdot \delta_{0, \operatorname{dim}_{\mathbb{C}} \mathcal{X}_{h}}
$$

Let $\mathcal{R}=H^{*}(\mathcal{B} \widetilde{T} ; \mathbb{C})=\mathbb{C}\left[\mathrm{w}_{1}, \mathrm{w}_{2}, \mathrm{w}_{3}\right]$, where $\mathrm{w}_{1}, \mathrm{w}_{2}, \mathrm{w}_{3}$ are the first Chern classes of the universal line bundles over $\mathcal{B} \widetilde{T}$. The $\widetilde{T}$-equivariant Chen-Ruan orbifold cohomology $H_{\mathrm{CR}, \widetilde{T}}^{*}\left(\left[\mathbb{C}^{3} / G\right] ; \mathbb{C}\right)$ is an $\mathcal{R}$-module. Given $h \in G$, define

$$
\mathbf{e}_{h}:=\prod_{i=1}^{3} \mathrm{w}_{i}^{\delta_{c_{i}(h), 0}} \in \mathcal{R} .
$$




\section{All-Genus OPEN-ClOSED MIRROR SYMMETRY}

In particular, $\mathbf{e}_{1}=\mathrm{w}_{1} \mathrm{w}_{2} \mathrm{w}_{3}$. Then the $\widetilde{T}$-equivariant Euler class of $\mathbf{0}_{h}:=[0 / G]$ in $\mathcal{X}_{h}=\left[\left(\mathbb{C}^{3}\right)^{h} / G\right]$ is

$$
e_{\widetilde{T}}\left(T_{\mathbf{0}_{h}} \mathcal{X}_{h}\right)=\mathbf{e}_{h} \mathbf{1}_{h} \in H_{\widetilde{T}}^{*}\left(\mathcal{X}_{h} ; \mathbb{C}\right)=\mathcal{R} \mathbf{1}_{h}
$$

Let $\chi_{1}, \chi_{2}, \chi_{3} \in G^{*}$ be defined as in Section 2.1. The image of $\chi_{i}: G \rightarrow \mathbb{C}^{*}$ is $\boldsymbol{\mu}_{r_{i}}$ for some positive integer $r_{i}$. In particular, $r_{1}=r$. Define

$$
\mathcal{R}^{\prime}=\mathbb{C}\left[\mathrm{w}_{1}^{1 / r_{1}}, \mathrm{w}_{2}^{1 / r_{2}}, \mathrm{w}_{3}^{1 / r_{3}}\right],
$$

which is a finite extension of $\mathcal{R}$. Let

$$
\mathcal{Q}=\mathbb{C}\left(\mathrm{w}_{1}, \mathrm{w}_{2}, \mathrm{w}_{3}\right) \quad \text { and } \quad \mathcal{Q}^{\prime}=\mathbb{C}\left(\mathrm{w}_{1}^{1 / r_{1}}, \mathrm{w}_{2}^{1 / r_{2}}, \mathrm{w}_{3}^{1 / r_{3}}\right)
$$

be the fractional fields of $\mathcal{R}$ and $\mathcal{R}^{\prime}$, respectively. The $\widetilde{T}$-equivariant Poincaré pairing of $H_{\widetilde{T}, \mathrm{CR}}^{*}(\mathcal{X} ; \mathbb{C}) \otimes_{\mathcal{R}} \mathcal{Q}$ (which is isomorphic to $H_{\mathrm{CR}}^{*}(\mathcal{X} ; \mathcal{Q})$ as a vector space over $\mathcal{Q}$ ) is given by

$$
\left\langle\mathbf{1}_{h}, \mathbf{1}_{h^{\prime}}\right\rangle_{\mathcal{X}}=\frac{1}{|G|} \cdot \frac{\delta_{h^{-1}, h^{\prime}}}{\mathbf{e}_{h}} \in \mathcal{Q} .
$$

The $\widetilde{T}$-equivariant orbifold cup product of $H_{\widetilde{T}, \mathrm{CR}}^{*}(\mathcal{X} ; \mathbb{C}) \otimes_{\mathcal{R}} \mathcal{Q}$ is given by

$$
\mathbf{1}_{h} \star \mathcal{X} \mathbf{1}_{h^{\prime}}=\left(\prod_{i=1}^{3} \mathrm{w}_{i}^{c_{i}(h)+c_{i}\left(h^{\prime}\right)-c_{i}\left(h h^{\prime}\right)}\right) \mathbf{1}_{h h^{\prime}} .
$$

Define

$$
\overline{\mathbf{1}}_{h}:=\frac{\mathbf{1}_{h}}{\prod_{i=1}^{3} \mathrm{w}_{i}^{c_{i}(h)}} \in H_{\widetilde{T}, \mathrm{CR}}^{*}(\mathcal{X} ; \mathbb{C}) \otimes_{\mathcal{R}} \mathcal{Q}^{\prime}
$$

Then

$$
\left\langle\overline{\mathbf{1}}_{h}, \overline{\mathbf{1}}_{h^{\prime}}\right\rangle_{\mathcal{X}}=\frac{\delta_{h^{-1}, h^{\prime}}}{|G| \mathrm{w}_{1} \mathrm{w}_{2} \mathrm{w}_{3}}, \quad \overline{\mathbf{1}}_{h} \star \mathcal{X} \overline{\mathbf{1}}_{h^{\prime}}=\overline{\mathbf{1}}_{h h^{\prime}} .
$$

We now define a canonical basis for the semi-simple algebra $H_{\widetilde{T}, \mathrm{CR}}^{*}(\mathcal{X} ; \mathbb{C}) \otimes_{\mathcal{R}} \mathcal{Q}^{\prime}$. Given $\gamma \in G^{*}$, define

$$
\bar{\phi}_{\gamma}:=\frac{1}{|G|} \sum_{h \in G} \chi_{\gamma}\left(h^{-1}\right) \overline{\mathbf{1}}_{h}
$$

Then

$$
\left\langle\bar{\phi}_{\gamma}, \bar{\phi}_{\gamma^{\prime}}\right\rangle_{\mathcal{X}}=\frac{\delta_{\gamma \gamma^{\prime}}}{|G|^{2} \mathrm{w}_{1} \mathrm{w}_{2} \mathrm{w}_{3}}, \quad \bar{\phi}_{\gamma} \star \mathcal{X} \bar{\phi}_{\gamma^{\prime}}=\delta_{\gamma \gamma^{\prime}} \bar{\phi}_{\gamma}
$$

So $\left\{\bar{\phi}_{\gamma}: \gamma \in G^{*}\right\}$ is a canonical basis of $H_{\widetilde{T}, \mathrm{CR}}^{*}(\mathcal{X} ; \mathbb{C}) \otimes_{\mathcal{R}} \mathcal{Q}^{\prime}$.

\subsection{Equivariant Chen-Ruan orbifold cohomology of $\mathcal{Y}_{\boldsymbol{f}}$}

We use the notation in Sections 3.2 and 3.3: the coarse moduli space $Y_{f}$ of the toric 3-orbifold $\mathcal{Y}_{f}$ is defined by a simplicial fan $\Sigma_{f}$ with two 3 -dimensional cones $\sigma$ and $\sigma^{\prime}$. Let $\mathcal{X}=\left[\mathbb{C}^{3} / G\right]$ and $\mathcal{X}^{\prime}=\left[\mathbb{C}^{3} / \boldsymbol{\mu}_{m}\right]$ be the affine toric sub-orbifolds of $\mathcal{Y}_{f}$ associated with $\sigma$ and $\sigma^{\prime}$, respectively. As $\mathcal{Q}$-vector spaces,

$$
H_{\widetilde{T}, \mathrm{CR}}^{*}\left(\mathcal{Y}_{f} ; \mathbb{C}\right) \otimes_{\mathcal{R}} \mathcal{Q}=H_{\mathrm{CR}}^{*}\left(\mathcal{Y}_{f} ; \mathcal{Q}\right)=H_{\mathrm{CR}}^{*}(\mathcal{X} ; \mathcal{Q}) \oplus H_{\mathrm{CR}}^{*}\left(\mathcal{X}^{\prime} ; \mathcal{Q}\right)
$$

where

$$
H_{\mathrm{CR}}^{*}(\mathcal{X} ; \mathcal{Q})=\bigoplus_{h \in G} \mathcal{Q} \mathbf{1}_{h}, \quad H_{\mathrm{CR}}^{*}\left(\mathcal{X}^{\prime} ; \mathcal{Q}\right)=\mathcal{Q} \mathbf{1}^{\prime} \oplus \bigoplus_{k=1}^{m-1} \mathcal{Q} \mathbf{1}_{k / m}^{\prime}
$$




\section{B. FAnG, C.-C. M. LiU And Z. ZonG}

We have the following isomorphisms of $\mathcal{Q}$-vector spaces (which do not preserve the grading and the orbifold cup product):

$$
\begin{aligned}
& H_{\mathrm{CR}}^{*}\left(\mathcal{Y}_{f} ; \mathcal{Q}\right) \cong H_{\mathrm{CR}}^{*}(\mathfrak{l} ; \mathcal{Q}), \quad H_{\mathrm{CR}}^{*}(\mathcal{X} ; \mathcal{Q}) \cong H_{\mathrm{CR}}^{*}(\mathcal{B} G ; \mathcal{Q}) \\
& H_{\mathrm{CR}}^{*}\left(\mathcal{X}^{\prime} ; \mathcal{Q}\right) \cong H_{\mathrm{CR}}^{*}\left(\mathcal{D}_{f} ; \mathcal{Q}\right) \cong H_{\mathrm{CR}}^{*}\left(\mathfrak{p}_{\infty} ; \mathcal{Q}\right)=H_{\mathrm{CR}}^{*}\left(\mathcal{B} \boldsymbol{\mu}_{m} ; \mathcal{Q}\right)
\end{aligned}
$$

\section{A-model topological string}

\subsection{Relative orbifold Gromov-Witten invariants of $\left(\mathcal{Y}_{f}, \mathcal{D}_{f}\right)$}

In this section, we define open-closed orbifold Gromov-Witten invariants of $\mathcal{X}$ as certain equivariant relative orbifold Gromov-Witten invariants of $\left(\mathcal{Y}_{f}, \mathcal{D}_{f}\right)$. Recall from Section 3.3 that the orbifold $\mathcal{Y}_{f}$ can be viewed as the total space of the rank 2 vector bundle $V=\mathcal{L}_{2} \oplus \mathcal{L}_{3} \rightarrow \mathfrak{l}$, where $\mathfrak{l}$ is a $\boldsymbol{\mu}_{m}$-gerbe over $\mathbb{P}(1, r)$, so equivariant relative orbifold Gromov-Witten invariants of $\left(\mathcal{Y}_{f}, \mathcal{D}_{f}\right)$ are twisted equivariant relative orbifold Gromov-Witten invariants of $\left(\mathfrak{l}, \mathfrak{p}_{\infty}\right)$.

Relative orbifold Gromov-Witten theory has been constructed in algebraic geometry by Abramovich-Fantechi [AF16] and in symplectic geometry by Chen-Li-Sun-Zhao [CLSZ11]. We refer to [AF16, CLSZ11] for the precise definitions of moduli spaces of relative stable maps to smooth DM stacks.

We first introduce some notation. We fix non-negative integers $g$ and $\ell$ and a vector $\vec{\mu}=$ $\left(\left(\mu_{1}, k_{1}\right), \ldots,\left(\mu_{n}, k_{n}\right)\right)$, where $\mu_{j} \in \mathbb{Z}_{>0}$ and $k_{j} \in\{0,1, \ldots, m-1\}$.

(1) Let $\overline{\mathcal{M}}_{g, \ell}\left(\mathcal{Y}_{f} / \mathcal{D}_{f}, \vec{\mu}\right)$ and $\overline{\mathcal{M}}_{g, \ell}\left(\mathfrak{l} / \mathfrak{p}_{\infty}, \vec{\mu}\right)$ be the moduli spaces of genus $g$ relative stable maps to $\left(\mathcal{Y}_{f}, \mathcal{D}_{f}\right)$ and $\left(\mathfrak{l}, \mathfrak{p}_{\infty}\right)$, respectively, with $\ell$ marked points and $n$ relative points, where the relative points are ordered, and the ramification index of the $j$ th relative point is $\mu_{j}$ and its monodromy is $k_{j}$.

(2) Let $\mathrm{ev}_{i}: \overline{\mathcal{M}}_{g, \ell}\left(\mathcal{Y}_{f} / \mathcal{D}_{f}, \vec{\mu}\right) \rightarrow \mathcal{I} \mathcal{Y}_{f}$ and $\mathrm{ev}_{i}: \overline{\mathcal{M}}_{g, \ell}\left(\mathfrak{l} / \mathfrak{p}_{\infty}, \vec{\mu}\right) \rightarrow \mathcal{I l}$ be the evaluation maps at the $i$ th marked point, where $i=1, \ldots, \ell$.

(3) Let $\pi: \mathcal{U} \rightarrow \overline{\mathcal{M}}_{g, \ell}\left(\mathfrak{l} / \mathfrak{p}_{\infty}, \vec{\mu}\right)$ be the universal curve, $\mathcal{T} \rightarrow \overline{\mathcal{M}}_{g, \ell}\left(\mathfrak{l} / \mathfrak{p}_{\infty}, \vec{\mu}\right)$ be the universal target, and $F: \mathcal{U} \rightarrow \mathcal{T}$ be the universal map.

(4) There is a contraction map $\mathcal{T} \rightarrow \mathfrak{l}$. Let $\hat{F}: \mathcal{U} \rightarrow \mathfrak{l}$ be the composition of this contraction map and the universal map $F: \mathcal{U} \rightarrow \mathcal{T}$.

(5) Let $D=\bigcup_{1 \leqslant j \leqslant n} D_{j} \subset \mathcal{U}$ be the universal relative points corresponding to $\vec{\mu}$, and let $D_{0}=$ $\underset{\substack{1 \leqslant j \leqslant n \\ k_{j}=0}}{\substack{1 \\ D_{j}}} D_{j} \subset D$.

(6) Let $\mathbb{T}_{f}=\left\{\left(t, t^{f}, 1\right): t \in \mathbb{C}^{*}\right\} \subset \mathbb{T} \cong\left(\mathbb{C}^{*}\right)^{3}$. This induces

$$
\begin{gathered}
\phi_{f}: H^{*}(B \mathbb{T} ; \mathbb{Q})=\mathbb{Q}\left[\mathrm{u}_{1}, \mathrm{u}_{2}, \mathrm{u}_{3}\right]=\mathbb{Q}\left[\mathrm{w}_{1}, \mathrm{w}_{2}, \mathrm{w}_{3}\right] \rightarrow H^{*}\left(B \mathbb{T}_{f} ; \mathbb{Q}\right)=\mathbb{Q}[\mathrm{v}], \\
\mathrm{u}_{1} \mapsto \mathrm{v}, \quad \mathrm{u}_{2} \mapsto f \mathrm{v}, \quad \mathrm{u}_{3} \mapsto 0
\end{gathered}
$$

or, equivalently,

$$
\mathrm{w}_{1} \mapsto \frac{1}{r} \mathrm{v}=w_{1} \mathrm{v}, \quad \mathrm{w}_{2} \mapsto \frac{s+r f}{r m} \mathrm{v}=w_{2} \mathrm{v}, \quad \mathrm{w}_{3} \mapsto \frac{-s-r f-m}{r m} \mathrm{v}=w_{3} \mathrm{v} .
$$

We extend $\phi_{f}$ to $\phi_{f}: \mathcal{Q}=\mathbb{C}\left(\mathrm{u}_{1}, \mathrm{u}_{2}, \mathrm{u}_{3}\right)=\mathbb{C}\left(\mathrm{w}_{1}, \mathrm{w}_{2}, \mathrm{w}_{3}\right) \rightarrow \mathbb{C}(\mathrm{v})$.

For any

$$
\gamma_{1}, \ldots, \gamma_{\ell} \in H_{\mathrm{CR}}^{*}(\mathcal{X} ; \mathcal{Q})=\bigoplus_{h \in G} \mathcal{Q} \mathbf{1}_{h} \subset H_{\mathrm{CR}}^{*}\left(\mathcal{Y}_{f} ; \mathcal{Q}\right) \cong H_{\mathrm{CR}}^{*}(\mathfrak{l} ; \mathcal{Q})
$$




\section{All-Genus OPEN-ClOSED MIRROR SYMMETRY}

we define the $\widetilde{T}$-equivariant relative orbifold Gromov-Witten invariant

$$
\begin{aligned}
\left\langle\gamma_{1}, \ldots, \gamma_{\ell}\right\rangle_{g, \vec{\mu}}^{\mathcal{X},(\mathcal{L}, f)} & :=\frac{1}{|\operatorname{Aut}(\vec{\mu})|} \cdot \phi_{f}\left(\int_{\left[\overline{\mathcal{M}}_{g, \ell}\left(\mathcal{X}_{f} / \mathcal{D}_{f}, \vec{\mu}\right)^{\widetilde{T}}\right]^{\mathrm{vir}}} \frac{\left(\mathrm{u}_{2}-f \mathrm{u}_{1}\right)^{\sum_{j=1}^{n} \delta_{k_{j}, 0}}}{e_{\widetilde{T}}\left(N^{\mathrm{vir}}\right)} \prod_{i=1}^{\ell} \mathrm{ev}_{i}^{*} \gamma_{i}\right) \\
& =\frac{1}{|\operatorname{Aut}(\vec{\mu})|} \cdot \phi_{f}\left(\int_{\left[\overline{\mathcal{M}}_{g, \ell}\left(\mathfrak{l} / \mathfrak{p}_{\infty}, \vec{\mu}\right)^{\widetilde{T}}\right]^{\mathrm{vir}}} \frac{e_{\widetilde{T}}\left(-R \pi_{*}\left(\hat{F}^{*} \mathcal{L}_{2}\left(-D_{0}\right) \oplus \hat{F}^{*} \mathcal{L}_{3}\right)\right)}{e_{\widetilde{T}}\left(N^{\mathrm{vir}}\right)} \prod_{i=1}^{\ell} \mathrm{ev}_{i}^{*} \gamma_{i}\right) \\
& \in \mathbb{Q}(\mathrm{v}) .
\end{aligned}
$$

Note that $\phi_{f}\left(\mathbf{u}_{2}-f \mathbf{u}_{1}\right)=0$. The factor $\left(\mathbf{u}_{2}-f \mathbf{u}_{1}\right)^{\sum_{j=1}^{n} \delta_{k_{j}, 0}}$ is included to cancel such a factor in $e_{\widetilde{T}}\left(N^{\text {vir }}\right)$.

If $\gamma_{1}, \ldots, \gamma_{\ell}$ are homogeneous, then

$$
\left\langle\gamma_{1}, \ldots, \gamma_{\ell}\right\rangle_{g, \vec{\mu}}^{\mathcal{X},(\mathcal{L}, f)}=\left.\mathrm{v}^{\sum_{i=1}^{\ell}\left(\operatorname{deg} \gamma_{i} / 2-1\right)} \cdot\left\langle\gamma_{1}, \ldots, \gamma_{\ell}\right\rangle_{g, \vec{\mu}}^{\mathcal{X},(\mathcal{L}, f)}\right|_{\mathrm{v}=1} .
$$

\subsection{Descendant orbifold Gromov-Witten invariants of $\mathcal{X}$}

In this subsection, we use localization to compute $\left\langle\gamma_{1}, \ldots, \gamma_{\ell}\right\rangle_{g, \vec{\mu}}^{\mathcal{X},(\mathcal{L}, f)}$ and obtain an expression in terms of cubic abelian Hurwitz-Hodge integrals.

We first give some definitions. For $i=1,2,3$, let $\chi_{i} \in G^{*}=\operatorname{Hom}\left(G, \mathbb{C}^{*}\right)$ be the characters of $G$ defined in Section 2.1, and let $L_{\chi_{i}}$ be the line bundle over $\mathcal{B} G$ associated with the irreducible character $\chi_{i} \in G^{*}$. Then $\mathcal{X}=\left[\mathbb{C}^{3} / G\right]$ is the total space of

$$
\bigoplus_{i=1}^{3} L_{\chi_{i}} \rightarrow \mathcal{B} G
$$

Let $\overline{\mathcal{M}}_{g, n}(\mathcal{B} G)$ denote the moduli space of genus $g, n$-pointed twisted stable maps to $\mathcal{B} G$. Let $\pi: \mathcal{C}_{g, n} \rightarrow \overline{\mathcal{M}}_{g, n}(\mathcal{B} G)$ be the universal curve, and let $F: \mathcal{C}_{g, n} \rightarrow \mathcal{B} G$ be the universal map. Define

$$
\mathbf{e}_{g, n}:=\phi_{f}\left(e_{\widetilde{T}}\left(R \pi_{*} F^{*}\left(\bigoplus_{i=1}^{3} L_{\chi_{i}}\right)\right)\right)=\prod_{i=1}^{3}\left(w_{i} \mathrm{v}\right)^{\left(\operatorname{rank} R \pi_{*} F^{*} L_{\chi_{i}}\right)} c_{1 / w_{i} \mathrm{v}}\left(R \pi_{*} F^{*} L_{\chi_{i}}\right),
$$

where $c_{t}(E)=1+t c_{1}(E)+t^{2} c_{2}(E)+\cdots$ denotes the Chern polynomial of a complex vector bundle $E$ (or, more generally, an element in the $K$-theory). The rank of $R \pi_{*} F^{*} L_{\chi_{i}}$ is constant on a connected component of $\overline{\mathcal{M}}_{g, n}(\mathcal{B} G)$ but might be different on different connected components of $\overline{\mathcal{M}}_{g, n}(\mathcal{B} G)$.

A moduli point in $\overline{\mathcal{M}}_{g, \ell}\left(\mathfrak{l} / \mathfrak{p}_{\infty}, \vec{\mu}\right)$ is represented by a morphism

$$
\rho:\left(\mathcal{C}, x_{1}, \ldots, x_{\ell}, y_{1}, \ldots, y_{n}\right) \rightarrow \mathfrak{l}[k]=\mathfrak{l} \cup \Delta_{1} \cup \cdots \cup \Delta_{k},
$$

where each $\Delta_{i}$ is a trivial $\boldsymbol{\mu}_{m}$-gerbe over $\mathbb{P}^{1}$. Let

$$
\overline{\mathcal{M}}_{g, \ell}^{0}\left(\mathfrak{l} / \mathfrak{p}_{\infty}, \vec{\mu}\right) \subset \overline{\mathcal{M}}_{g, \ell}\left(\mathfrak{l} / \mathfrak{p}_{\infty}, \vec{\mu}\right)
$$

be the substack where the target is $\mathfrak{l}[0]=\mathfrak{l}$. Computations in [RZ13] show that the contribution from a $\widetilde{T}$-fixed locus outside $\overline{\mathcal{M}}_{g, \ell}^{0}\left(\mathfrak{l} / \mathfrak{p}_{\infty}, \vec{\mu}\right)$ vanishes. Therefore,

$$
\left\langle\gamma_{1}, \ldots, \gamma_{\ell}\right\rangle_{g, \vec{\mu}}^{\mathcal{X},(\mathcal{L}, f)}=\frac{1}{|\operatorname{Aut}(\vec{\mu})|} \cdot \phi_{f}\left(\int_{\left[\overline{\mathcal{M}}_{g, \ell}^{0}\left(\mathfrak{l} / \mathfrak{p}_{\infty}, \vec{\mu}\right)^{T}\right] \text { vir }} \frac{e_{\widetilde{T}}\left(-R \pi_{*}\left(\hat{F}^{*} \mathcal{L}_{2}\left(-D_{0}\right) \oplus \hat{F}^{*} \mathcal{L}_{3}\right)\right)}{e_{\widetilde{T}}\left(N^{\mathrm{vir}}\right)} \prod_{i=1}^{\ell} \operatorname{ev}_{i}^{*} \gamma_{i}\right) .
$$


Suppose that $\rho:\left(\mathcal{C}, x_{1}, \ldots, x_{\ell}, y_{1}, \ldots, y_{n}\right) \rightarrow \mathfrak{l}$ represents a point in $\overline{\mathcal{M}}_{g, n+\ell}^{0}\left(\mathfrak{l} / \mathfrak{p}_{\infty}, \vec{\mu}\right)^{\widetilde{T}}$. Then

$$
\mathcal{C}=\mathcal{C}_{0} \cup \bigcup_{j=1}^{n} \mathcal{C}_{j}
$$

where $\mathcal{C}_{0}$ is an orbicurve of genus $g$, the components $\mathcal{C}_{1}, \ldots, \mathcal{C}_{n}$ are 1-dimensional toric orbifolds, $x_{1}, \ldots, x_{\ell} \in \mathcal{C}_{0}$ and $y_{j} \in \mathcal{C}_{j}$, and $\mathcal{C}_{0}$ and $\mathcal{C}_{j}$ intersect at a node $z_{j}$ for $j=1, \ldots, n$. We view $z_{j}$ as a point on $\mathcal{C}_{j}$ in order to determine its monodromy. Let $\rho_{j}:=\left.\rho\right|_{\mathcal{C}_{j}}: \mathcal{C}_{j} \rightarrow \mathfrak{l}$. Then $\rho_{0}$ is a constant map to $\mathfrak{p}_{0}=[0,1]$, and we have the following diagram for $1 \leqslant j \leqslant n$ :

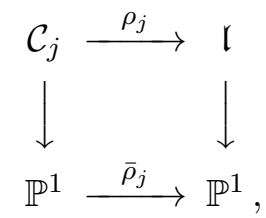

where $\bar{\rho}_{j}([x, y])=\left[x^{\mu_{j}}, y^{\mu_{j}}\right]$ is the map between coarse moduli spaces, the monodromy around $y_{j}$ is $e^{2 \pi \sqrt{-1} k_{j} / m} \in \boldsymbol{\mu}_{m}$, and the monodromy around $z_{j}$ is

$$
\left(e^{2 \pi \sqrt{-1} \mu_{j} w_{1}}, e^{2 \pi \sqrt{-1}\left(\mu_{j} w_{2}-k_{j} / m\right)}, e^{2 \pi \sqrt{-1}\left(\mu_{j} w_{3}+k_{j} / m\right)}\right)=\eta_{1}^{\mu_{j}} \eta_{2}^{-k_{j}} \in G .
$$

For $\left(d_{0}, k\right) \in \mathbb{Z} \times \mathbb{Z}_{m}$, define $h\left(d_{0}, k\right):=\eta_{1}^{d_{0}} \eta_{2}^{-k} \in G$, and define $D^{\prime}\left(d_{0}, k\right)$ to be

$$
-\frac{r}{\mathrm{v}}(-1)^{\left\lfloor d_{0} w_{3}+k / m\right\rfloor}\left(\frac{\mathrm{v}}{d_{0}}\right)^{\operatorname{age}\left(h\left(d_{0}, k\right)\right)-1} \cdot \frac{\Gamma\left(d_{0}\left(w_{1}+w_{2}\right)+c_{3}\left(h\left(d_{0}, k\right)\right)\right)}{\Gamma\left(d_{0} w_{1}-c_{1}\left(h\left(d_{0}, k\right)\right)+1\right) \Gamma\left(d_{0} w_{2}-c_{2}\left(h\left(d_{0}, k\right)\right)+1\right)} .
$$

Then $D^{\prime}\left(d_{0}, k\right) \in \mathbb{C v}^{\text {age }}\left(h\left(d_{0}, k\right)\right)-2$. Up to the sign, $D^{\prime}\left(d_{0}, k\right)$ is essentially $|G|$ times the disk function in [Ros14, Section 3.3]. Note that

$$
(-1)^{\left\lfloor d_{0} w_{3}+k / m\right\rfloor}=(-1)^{d_{0} w_{3}-c_{3}\left(h\left(d_{0}, k\right)\right)+k / m} .
$$

By virtual localization, we have the following result.

Proposition 5.1. We have

$$
\begin{aligned}
\langle\rangle_{0,\left(d_{0}, k\right)}^{\mathcal{X},(\mathcal{L}, f)}= & \frac{\delta_{1, h\left(d_{0}, k\right)}}{|G|} D^{\prime}\left(d_{0}, k\right) \cdot\left(\frac{\mathrm{v}}{d_{0}}\right)^{2}, \\
\langle\gamma\rangle_{0,\left(d_{0}, k\right)}^{\mathcal{X},(\mathcal{L}, f)}= & \left\langle\gamma, \mathbf{e}_{h\left(d_{0}, k\right)} \mathbf{1}_{h\left(d_{0}, k\right)^{-1}}\right\rangle_{\mathcal{X}} \cdot D^{\prime}\left(d_{0}, k\right) \cdot \frac{\mathrm{v}}{d_{0}}, \\
\langle\rangle_{0,\left(\mu_{1}, k_{1}\right),\left(\mu_{2}, k_{2}\right)}^{\mathcal{X},(\mathcal{L}, f)}= & \left\langle\mathbf{e}_{h\left(\mu_{1}, k_{1}\right)} \mathbf{1}_{\left.h\left(\mu_{1}, k_{1}\right)^{-1}, \mathbf{e}_{h\left(\mu_{2}, k_{2}\right)} \mathbf{1}_{h\left(\mu_{2}, k_{2}\right)^{-1}}\right\rangle_{\mathcal{X}}}\right. \\
& \cdot \frac{D^{\prime}\left(\mu_{1}, k_{1}\right) D^{\prime}\left(\mu_{2}, k_{2}\right)}{\left|\operatorname{Aut}\left(\left(\mu_{1}, k_{1}\right),\left(\mu_{2}, k_{2}\right)\right)\right|} \frac{\mathrm{v}}{\mu_{1}+\mu_{2}} .
\end{aligned}
$$

If $\vec{\mu}=\left(\left(\mu_{1}, k_{1}\right), \ldots,\left(\mu_{n}, k_{n}\right)\right)$ and $2 g-2+\ell+n>0$, then

$$
\left\langle\gamma_{1}, \ldots, \gamma_{\ell}\right\rangle_{g, \vec{\mu}}^{\mathcal{X},(\mathcal{L}, f)}=\frac{1}{|\operatorname{Aut}(\vec{\mu})|} \prod_{j=1}^{n} D^{\prime}\left(\mu_{j}, k_{j}\right) \int_{\overline{\mathcal{M}}_{g, \ell+n}(B G)} \frac{\prod_{i=1}^{\ell} \mathrm{ev}_{i}^{*} \gamma_{i}}{\mathbf{e}_{g, \ell+n}} \prod_{j=1}^{n} \frac{\mathrm{ev}_{\ell+j}^{*}\left(\mathbf{e}_{h\left(\mu_{j}, k_{j}\right)} \mathbf{1}_{h\left(\mu_{j}, k_{j}\right)^{-1}}\right)}{1-\left(\mu_{j} / \mathrm{v}\right) \bar{\psi}_{\ell+j}} .
$$

Introduce variables $X_{1}, \ldots, X_{n}$, and let

$$
\boldsymbol{\tau}=\sum_{a=1}^{p} \tau_{a} \mathbf{1}_{h_{a}}
$$




\section{All-GenUs OPEN-CLOSED MIRROR SYMMETRY}

where $h_{a} \in G$ corresponds to $\left(m_{a}, n_{a}, 1\right) \in\{v \in \operatorname{Box}(\sigma)$ : age $(v)=1\}$. For $k \in\{0,1, \ldots, m-1\}$, let $\mathbf{1}_{-k / m}^{\prime}:=\mathbf{1}_{1-\delta_{0, k}-k / m}^{\prime} \in H_{\mathrm{CR}}^{*}\left(\mathcal{B} \boldsymbol{\mu}_{m} ; \mathbb{C}\right)=\bigoplus_{k=0}^{m-1} \mathbb{C} \mathbf{1}_{k / m}^{\prime}$. Define

$$
\begin{aligned}
F_{g, n}^{\mathcal{X},(\mathcal{L}, f)}\left(\boldsymbol{\tau} ; X_{1}, \ldots, X_{n}\right)= & \sum_{\mu_{1}, \ldots, \mu_{n}>0} \sum_{k_{1}, \ldots, k_{n}=0}^{m-1} \sum_{\ell \geqslant 0} \frac{\left\langle\boldsymbol{\tau}^{\ell}\right\rangle_{g,\left(\mu_{1}, k_{1}\right), \ldots,\left(\mu_{n}, k_{n}\right)}^{\mathcal{X},(\mathcal{L}, f)}}{\ell !} \\
& \cdot \prod_{j=1}^{n}\left(X_{j}\right)^{\mu_{j}} \cdot(-1)^{-k_{1} / m} \mathbf{1}_{-k_{1} / m}^{\prime} \otimes \cdots \otimes(-1)^{-k_{n} / m} \mathbf{1}_{-k_{n} / m}^{\prime},
\end{aligned}
$$

which is an $H_{\mathrm{CR}}^{*}\left(\mathcal{B} \boldsymbol{\mu}_{m} ; \mathbb{C}\right)^{\otimes n}$-valued function, where $H_{\mathrm{CR}}^{*}\left(\mathcal{B} \boldsymbol{\mu}_{m} ; \mathbb{C}\right)=\bigoplus_{k=0}^{m-1} \mathbb{C} \mathbf{1}_{k / m}^{\prime}$.

Remark 5.2. For each $k \in\{0,1, \ldots, m-1\}$, the set $\left\{\mu_{j}: k_{j}=k\right\}$ determines a partition $\mu^{k}=$ $\left(\mu_{1}^{k} \geqslant \cdots \geqslant \mu_{\ell\left(\mu^{k}\right)}^{k}>0\right)$. We have

$$
\begin{aligned}
& \operatorname{Aut}\left(\left(\mu_{1}, k_{1}\right), \ldots,\left(\mu_{n}, k_{n}\right)\right)=\prod_{k=0}^{m-1} \operatorname{Aut}\left(\mu^{k}\right), \\
& \ell\left(\mu^{0}\right)+\cdots+\ell\left(\mu^{m-1}\right)=n .
\end{aligned}
$$

The correlator $\left\langle\boldsymbol{\tau}^{\ell}\right\rangle_{g,\left(\mu_{1}, k_{1}\right), \ldots,\left(\mu_{n}, k_{n}\right)}^{\mathcal{X},(\mathcal{L}, f)}$ is invariant under permutation of $\left(\mu_{1}, k_{1}\right), \ldots,\left(\mu_{n}, k_{n}\right)$, so it depends only on the $m$-tuple of partitions $\mu^{0}, \ldots, \mu^{m-1}$. So $F_{g, n}^{\mathcal{X},(\mathcal{L}, f)}\left(\boldsymbol{\tau} ; X_{1}, \ldots, X_{n}\right)$ is a symmetric function in $X_{1}, \ldots, X_{n}$ and can be rewritten as

$$
F_{g, n}^{\mathcal{X},(\mathcal{L}, f)}\left(\boldsymbol{\tau} ; X_{1}, \ldots, X_{n}\right)=\sum_{\substack{\mu^{0}, \ldots, \mu^{m-1} \in \mathcal{P} \\ \ell\left(\mu^{0}\right)+\cdots+\ell\left(\mu^{m-1}\right)=n}} \sum_{\ell \geqslant 0} \frac{\left\langle\boldsymbol{\tau}^{\ell}\right\rangle_{g, \mu^{0}, \ldots, \mu^{m-1}}^{\mathcal{X}(\mathcal{L}, f)}}{\ell !} X_{\mu^{0}, \ldots, \mu^{m-1}},
$$

where $\mathcal{P}$ is the set of partitions and

$$
X_{\mu^{0}, \ldots, \mu^{m-1}}=\sum_{\sigma \in S_{n}} \prod_{k=0}^{m-1} \prod_{j=\ell\left(\mu^{0}\right)+\cdots+\ell\left(\mu^{k-1}\right)+1}^{\ell\left(\mu^{0}\right)+\cdots+\ell\left(\mu^{k}\right)} X_{\sigma(j)}^{\mu_{j}^{k}}\left((-1)^{-k / m} \mathbf{1}_{-k / m}^{\prime}\right)^{\otimes \ell\left(\mu^{k}\right)} .
$$

We introduce some notation.

(1) Given $h \in G$, define

$$
\begin{aligned}
\Phi_{0}^{h}(X):= & \frac{1}{|G|} \sum_{\substack{\left(d_{0}, k\right) \in \mathbb{Z}_{\geqslant 0} \times \mathbb{Z}_{m} \\
h\left(d_{0}, k\right)=h}} D^{\prime}\left(d_{0}, k\right) X^{d_{0}}(-1)^{-k / m} \mathbf{1}_{-k / m}^{\prime} \\
=- & \sum_{\substack{\left(d_{0}, k\right) \in \mathbb{Z}_{\geqslant 0} \times \mathbb{Z}_{m} \\
h\left(d_{0}, k\right)=h}} \frac{1}{m v} e^{\sqrt{-1} \pi\left(d_{0} w_{3}-c_{3}(h)\right)}\left(\frac{\mathrm{v}}{d_{0}}\right)^{\operatorname{age}(h)-1} \\
& \cdot \frac{\Gamma\left(d_{0}\left(w_{1}+w_{2}\right)+c_{3}(h)\right)}{\Gamma\left(d_{0} w_{1}-c_{1}(h)+1\right) \Gamma\left(d_{0} w_{2}-c_{2}(h)+1\right)} X^{d_{0}} \mathbf{1}_{-k / m}^{\prime} .
\end{aligned}
$$

Then $\Phi_{0}^{h}(X)$ takes values in $\bigoplus_{k=0}^{m-1} \mathbb{C v}^{\text {age }(v)-2} \mathbf{1}_{k / m}^{\prime}$. For $a \in \mathbb{Z}$ and $h \in G$, we define

$$
\Phi_{a}^{h}(X):=\frac{1}{|G|} \sum_{\substack{d_{0}>0 \\ h\left(d_{0}, k\right)=h}} D^{\prime}\left(d_{0}, k\right)\left(\frac{d_{0}}{\mathrm{v}}\right)^{a} X^{d_{0}}(-1)^{-k / m} \mathbf{1}_{-k / m}^{\prime} .
$$


Then $\Phi_{a}^{h}(X)$ takes values in $\bigoplus_{k=0}^{m-1} \mathbb{C}$ age $(v)-2-a \mathbf{1}_{k / m}^{\prime}$, and

$$
\Phi_{a+1}^{h}(X)=\left(\frac{1}{\mathrm{v}} X \frac{d}{d X}\right) \Phi_{a}^{h}(X) .
$$

(2) For $a \in \mathbb{Z}$ and $\gamma \in G^{*}$, we define

$$
\widetilde{\xi}_{a}^{\gamma}(X):=|G| \sum_{h \in G} \chi_{\gamma}\left(h^{-1}\right)\left(\prod_{i=1}^{3}\left(w_{i} \vee\right)^{1-c_{i}(h)}\right) \Phi_{a}^{h}(X) .
$$

Then $\widetilde{\xi}_{a}^{\gamma}(X)$ takes values in $\bigoplus_{k=0}^{m-1} \mathbb{C v}^{1-a} \mathbf{1}_{k / m}^{\prime}$.

(3) Given $\gamma_{1}, \gamma_{2} \in H_{\mathrm{CR}}^{*}(\mathcal{X} ; \mathbb{C}) \otimes_{\mathbb{C}} \mathbb{C}(\mathrm{v})$ and $a_{1}, a_{2} \in \mathbb{Z}_{\geqslant 0}$, define

$$
\begin{gathered}
\left\langle\tau_{a_{1}}\left(\gamma_{1}\right)\right\rangle_{0,1}^{\mathcal{X}, \mathbb{T}_{f}}=\delta_{a_{1}, 0}\left\langle 1, \gamma_{1}\right\rangle \mathcal{X} \cdot \mathrm{v}^{2}, \\
\left\langle\tau_{a_{1}}\left(\gamma_{1}\right) \tau_{a_{2}}\left(\gamma_{2}\right)\right\rangle_{0,2}^{\mathcal{X}, \mathbb{T}_{f}}=\delta_{a_{1}, 0} \delta_{a_{2}, 0} \cdot \mathrm{v}\left\langle\gamma_{1}, \gamma_{2}\right\rangle \mathcal{X} .
\end{gathered}
$$

(4) Given $\gamma_{1}, \ldots, \gamma_{n} \in H_{\mathrm{CR}}^{*}(\mathcal{X} ; \mathbb{C}) \otimes \mathbb{C}(\mathrm{v})$ and $a_{1}, \ldots, a_{n} \in \mathbb{Z}_{\geqslant 0}$, define

$$
\left\langle\tau_{a_{1}}\left(\gamma_{1}\right) \cdots \tau_{a_{n}}\left(\gamma_{n}\right)\right\rangle_{g, n}^{\mathcal{X}, \mathbb{T}_{f}}=\int_{\overline{\mathcal{M}}_{g, n}(\mathcal{B} G)} \frac{1}{\mathbf{e}_{g, n}} \prod_{i=1}^{n}\left(\operatorname{ev}_{i}^{*}\left(\gamma_{i}\right) \bar{\psi}_{i}^{a_{i}}\right) .
$$

(5) Given $h \in G$, define $\mathbf{1}_{h}^{*}=|G| \phi_{f}\left(\mathbf{e}_{h} \mathbf{1}_{h^{-1}}\right)$.

(6) In the rest of this paper, we consider $\mathbb{T}_{f}$-equivariant cohomology and write $\phi_{\gamma}$ and $\bar{\phi}_{\gamma}$ instead of $\phi_{f}\left(\phi_{\gamma}\right)$ and $\phi_{f}\left(\bar{\phi}_{\gamma}\right)$, respectively. (Recall that $\phi_{f}\left(\mathrm{w}_{i}\right)=w_{i} \mathrm{v}$.)

Proposition 5.3. We have the following equations:

(i) (Disk invariants)

$$
\begin{aligned}
F_{0,1}^{\mathcal{X},(\mathcal{L}, f)}(\boldsymbol{\tau} ; X)= & \Phi_{-2}^{1}(X)+\sum_{a=1}^{p} \tau_{a} \Phi_{-1}^{h_{a}}(X)+\sum_{a \in \mathbb{Z}_{\geqslant 0}} \sum_{h \in G} \sum_{\ell \geqslant 2} \frac{\left\langle\boldsymbol{\tau}^{\ell}, \tau_{a}\left(\mathbf{1}_{h}^{*}\right)\right\rangle_{0, \ell+1}^{\mathcal{X}, \mathbb{T}_{f}}}{\ell !} \Phi_{a}^{h}(X) \\
= & \left.\frac{1}{|G|^{2} w_{1} w_{2} w_{3}}\left(\sum_{\gamma \in G^{*}} \widetilde{\xi}_{-2}^{\gamma}(X)+\sum_{a=1}^{p} \tau_{a} \prod_{i=1}^{3} w_{i}^{c_{i}\left(h_{a}\right)} \sum_{\gamma \in G^{*}} \chi_{\gamma}\left(h_{a}\right) \widetilde{\xi}_{-1}^{\gamma}(X)\right)\right|_{\boldsymbol{v}=1} \\
& +\sum_{a \in \mathbb{Z}_{\geqslant 0}} \sum_{\gamma \in G^{*}} \sum_{\ell \geqslant 2} \frac{\left\langle\boldsymbol{\tau}^{\ell}, \tau_{a}\left(\bar{\phi}_{\gamma}\right)\right\rangle_{0, \ell+1}^{\mathcal{X}, \mathbb{T}_{f}}}{\ell !} \widetilde{\xi}_{a}^{\gamma}(X) .
\end{aligned}
$$

(ii) (Annulus invariants)

$$
\begin{aligned}
& F_{0,2}^{\mathcal{X},(\mathcal{L}, f)}\left(\boldsymbol{\tau} ; X_{1}, X_{2}\right)-F_{0,2}^{\mathcal{X},(\mathcal{L}, f)}\left(0 ; X_{1}, X_{2}\right) \\
& =\sum_{a_{1}, a_{2} \in \mathbb{Z}_{\geqslant 0}} \sum_{h_{1}, h_{2} \in G} \sum_{\ell \geqslant 1} \frac{\left\langle\boldsymbol{\tau}^{\ell}, \tau_{a_{1}}\left(\mathbf{1}_{h_{1}}^{*}\right) \tau_{a_{2}}\left(\mathbf{1}_{h_{2}}^{*}\right)\right\rangle_{0, \ell+2}^{\mathcal{X}, \mathbb{T}_{f}}}{\ell !} \Phi_{a_{1}}^{h_{1}}\left(X_{1}\right) \Phi_{a_{2}}^{h_{2}}\left(X_{2}\right) \\
& =\sum_{a_{1}, a_{2} \in \mathbb{Z}_{\geqslant 0}} \sum_{\gamma_{1}, \gamma_{2} \in G^{*}} \sum_{\ell \geqslant 1} \frac{\left\langle\boldsymbol{\tau}^{\ell}, \tau_{a_{1}}\left(\bar{\phi}_{\gamma_{1}}\right) \tau_{a_{2}}\left(\bar{\phi}_{\gamma_{2}}\right)\right\rangle_{0, \ell+2}^{\mathcal{X}, \mathbb{T}_{f}}}{\ell !} \widetilde{\xi}_{a_{1}}^{\gamma_{1}}\left(X_{1}\right) \widetilde{\xi}_{a_{2}}^{\gamma_{2}}\left(X_{2}\right),
\end{aligned}
$$




\section{All-Genus open-ClOSED MIRROR SYMMETRY}

where

$$
\begin{aligned}
& \left(X_{1} \frac{\partial}{\partial X_{1}}+X_{2} \frac{\partial}{\partial X_{2}}\right) F_{0,2}^{\mathcal{X},(\mathcal{L}, f)}\left(0 ; X_{1}, X_{2}\right)=\left.|G|\left(\sum_{h \in G} \mathbf{e}_{h} \Phi_{0}^{h}\left(X_{1}\right) \Phi_{0}^{h^{-1}}\left(X_{2}\right)\right)\right|_{\mathrm{v}=1} \\
& =\left.\frac{1}{|G|^{2} w_{1} w_{2} w_{3}}\left(\sum_{\gamma \in G^{*}} \widetilde{\xi}_{0}^{\gamma}\left(X_{1}\right) \widetilde{\xi}_{0}^{\gamma}\left(X_{2}\right)\right)\right|_{\mathrm{v}=1} .
\end{aligned}
$$

(iii) For $2 g-2+n>0$,

$$
\begin{aligned}
& F_{g, n}^{\mathcal{X},(\mathcal{L}, f)}\left(\boldsymbol{\tau} ; X_{1}, \ldots, X_{n}\right) \\
& =\sum_{a_{1}, \ldots, a_{n} \in \mathbb{Z}_{\geqslant 0}} \sum_{h_{1}, \ldots, h_{n} \in G} \sum_{\ell \geqslant 0} \frac{\left\langle\boldsymbol{\tau}^{\ell}, \tau_{a_{1}}\left(\mathbf{1}_{h_{1}}^{*}\right) \cdots \tau_{a_{n}}\left(\mathbf{1}_{h_{n}}^{*}\right)\right\rangle_{g, \ell+n}^{\mathcal{X}, \mathbb{T}_{f}}}{\ell !} \prod_{j=1}^{n} \Phi_{a_{j}}^{h_{j}}\left(X_{j}\right) \\
& =\sum_{a_{1}, \ldots, a_{n} \in \mathbb{Z}_{\geqslant 0}} \sum_{\gamma_{1}, \ldots, \gamma_{n} \in G^{*}} \sum_{\ell \geqslant 0} \frac{\left\langle\boldsymbol{\tau}^{\ell}, \tau_{a_{1}}\left(\bar{\phi}_{\gamma_{1}}\right) \cdots \tau_{a_{n}}\left(\bar{\phi}_{\gamma_{n}}\right)\right\rangle_{g, \ell+n}^{\mathcal{X}, \mathbb{T}_{f}}}{\ell !} \prod_{j=1}^{n} \widetilde{\xi}_{a_{j}}^{\gamma_{j}}\left(X_{j}\right) .
\end{aligned}
$$

Remark 5.4. The function $F_{0,2}^{\mathcal{X},(\mathcal{L}, f)}\left(0 ; X_{1}, X_{2}\right)$ is an $H^{*}\left(\mathcal{B} \boldsymbol{\mu}_{m} ; \mathbb{C}\right)^{\otimes 2}$-valued power series in $X_{1}$ and $X_{2}$ which vanishes at $\left(X_{1}, X_{2}\right)=(0,0)$, so it is determined by $(5.5)$.

\subsection{A-model graph sum}

Introduce formal variables

$$
\hat{\mathbf{u}}=\sum_{a \geqslant 0} \hat{u}_{a} z^{a}=\sum_{a \geqslant 0} \sum_{\beta \in G^{*}} u_{a}^{\beta} \bar{\phi}_{\beta} z^{a}=\sum_{\beta \in G^{*}} \mathbf{u}^{\beta}(z) \bar{\phi}_{\beta},
$$

where

$$
u_{a}^{\beta} \in \mathcal{Q}, \quad \hat{u}_{a}=\sum_{\beta \in G^{*}} u_{a}^{\beta} \bar{\phi}_{\beta} \in H_{\mathrm{CR}}^{*}(\mathcal{X} ; \mathcal{Q}), \quad \mathbf{u}^{\beta}(z)=\sum_{a \geqslant 0} u_{a}^{\beta} z^{a} .
$$

For $j=1, \ldots, n$, define $\hat{\mathbf{u}}_{j}$ and $\mathbf{u}_{j}^{\beta}(z)$ similarly. Define

$$
\left\langle\hat{\mathbf{u}}^{\ell}, \hat{\mathbf{u}}_{1}, \ldots, \hat{\mathbf{u}}_{n}\right\rangle_{g, \ell+n}^{\mathcal{X}, \mathbb{T}_{f}}=\sum_{a_{1}, \ldots, a_{\ell}, b_{1}, \ldots, b_{n} \in \mathbb{Z}_{\geqslant 0}}\left\langle\tau_{a_{1}}\left(\hat{u}_{a_{1}}\right) \cdots \tau_{a_{\ell}}\left(\hat{u}_{a_{\ell}}\right) \tau_{b_{1}}\left(\left(\hat{u}_{1}\right)_{b_{1}}\right) \cdots \tau_{b_{n}}\left(\left(\hat{u}_{n}\right)_{b_{n}}\right)\right\rangle_{g, \ell+n}^{\mathcal{X}, \mathbb{T}_{f}} .
$$

By [FLZ16, Theorem 4.2], the term $(1 / \ell !)\left\langle\hat{\mathbf{u}}^{\ell}, \hat{\mathbf{u}}_{1}, \ldots, \hat{\mathbf{u}}_{n}\right\rangle_{g, \ell+n}^{\mathcal{X}, \mathbb{T}_{f}}$ can be written as a graph sum. By Proposition 5.3,

$$
F_{g, n}^{\mathcal{X},(\mathcal{L}, f)}\left(\boldsymbol{\tau} ; X_{1}, \ldots, X_{n}\right)=\sum_{\ell \geqslant 0} \frac{1}{\ell !}\left\langle\boldsymbol{\tau}^{\ell}, \widetilde{\xi}\left(X_{1}\right), \ldots, \widetilde{\xi}\left(X_{n}\right)\right\rangle_{g, \ell+n}^{\mathcal{X}, \mathbb{T}_{f}},
$$

where $\widetilde{\xi}(X)=\sum_{a \in \mathbb{Z} \geqslant 0} \sum_{\beta \in G^{*}} \widetilde{\xi}_{a}^{\beta}(X) \bar{\phi}_{\beta} z^{a}$. Therefore, a graph sum of $F_{g, n}^{\mathcal{X},(\mathcal{L}, f)}\left(\boldsymbol{\tau} ; X_{1}, \ldots, X_{n}\right)$ can be obtained by the following specialization of [FLZ16, Theorem 2]:

$$
\hat{\mathbf{u}}=\boldsymbol{\tau}=\sum_{a=1}^{p} \tau_{a} \mathbf{1}_{h_{a}}, \quad \hat{\mathbf{u}}_{j}=\widetilde{\xi}\left(X_{j}\right), \quad \mathrm{w}_{i}=w_{i} \vee .
$$

By (5.1), we may let $\mathbf{v}=1$; that is, $\mathbf{w}_{i}=w_{i}$. In the rest of this subsection, we give the precise statement of this graph sum.

Given a connected graph $\Gamma$, we introduce the following notation:

(1) $V(\Gamma)$ is the set of vertices in $\Gamma$. 


\section{B. FAnG, C.-C. M. LiU And Z. ZonG}

(2) $E(\Gamma)$ is the set of edges in $\Gamma$.

(3) $H(\Gamma)$ is the set of half-edges in $\Gamma$.

(4) $L^{O}(\Gamma)$ is the set of open leaves in $\Gamma$. The open leaves are ordered: $L^{O}(\Gamma)=\left\{l_{1}, \ldots, l_{n}\right\}$, where $n$ is the number of open leaves. (Open leaves correspond to ordered ordinary leaves in $[$ FLZ16].)

(5) $L^{o}(\Gamma)$ is the set of primary leaves in $\Gamma$. The primary leaves are unordered. (Primary leaves correspond to unordered ordinary leaves in [FLZ16].)

(6) $L^{1}(\Gamma)$ is the set of dilaton leaves in $\Gamma$. The dilaton leaves are not ordered.

With the above notation, we introduce the following labels:

(1) (genus) $g: V(\Gamma) \rightarrow \mathbb{Z}_{\geqslant 0}$,

(2) (marking) $\alpha: V(\Gamma) \rightarrow G^{*}$ (This induces

$$
\alpha: L(\Gamma)=L^{O}(\Gamma) \cup L^{o}(\Gamma) \cup L^{1}(\Gamma) \rightarrow G^{*}
$$

as follows: if $l \in L(\Gamma)$ is a leaf attached to a vertex $v \in V(\Gamma)$, define $\alpha(l)=\alpha(v)$.),

(3) (height) $k: H(\Gamma) \rightarrow \mathbb{Z}_{\geqslant 0}$.

Given a vertex $v \in V(\Gamma)$, let $H(v)$ denote the set of half-edges emanating from $v$. The the valency $\operatorname{val}(v)$ of the vertex $v$ is equal to the size $|H(v)|$ of the set $H(v)$. A labeled graph $\vec{\Gamma}=(\Gamma, g, \alpha, k)$ is stable if $2 g(v)-2+\operatorname{val}(v)>0$ for all $v \in V(\Gamma)$.

Let $\boldsymbol{\Gamma}(\mathcal{B} G)$ denote the set of all stable labeled graphs $\vec{\Gamma}=(\Gamma, g, \alpha, k)$. The genus of a stable labeled graph $\vec{\Gamma}$ is defined to be

$$
g(\vec{\Gamma}):=\sum_{v \in V(\Gamma)} g(v)+|E(\Gamma)|-|V(\Gamma)|+1=\sum_{v \in V(\Gamma)}(g(v)-1)+\sum_{e \in E(\Gamma)} 1+1 .
$$

Define

$$
\Gamma_{g, \ell, n}(\mathcal{B} G)=\left\{\vec{\Gamma}=(\Gamma, g, \alpha, k) \in \Gamma(\mathcal{B} G): g(\vec{\Gamma})=g,\left|L^{o}(\Gamma)\right|=\ell,\left|L^{O}(\Gamma)\right|=n\right\} .
$$

Let

$$
R(z)=\prod_{i=1}^{3} \exp \left(\sum_{m \geqslant 1} \frac{(-1)^{m}}{m(m+1)} A_{m+1}^{i}\left(\frac{z}{w_{i}}\right)^{m}\right)
$$

where $A_{m}^{i}$ is the operator defined by

$$
A_{m}^{i}: H^{*}(\mathcal{I B} G ; \mathbb{C}) \rightarrow H^{*}(\mathcal{I B} G ; \mathbb{C}), \quad \mathbf{1}_{h} \mapsto B_{m}\left(c_{i}(h)\right) \mathbf{1}_{h}
$$

and $B_{m}(x)$ is the Bernoulli polynomial. Then, relative to the basis $\left\{\phi_{\gamma}: \gamma \in G^{*}\right\}$,

$$
R(z)_{\beta}^{\alpha}=\frac{1}{|G|} \sum_{h \in G} \chi_{\alpha}(h) \chi_{\beta}\left(h^{-1}\right) \prod_{i=1}^{3} \exp \left(\sum_{m=1}^{\infty} \frac{(-1)^{m}}{m(m+1)} B_{m+1}\left(c_{i}(h)\right)\left(\frac{z}{w_{i}}\right)^{m}\right) .
$$

Given $\beta \in G^{*}$, define

$$
\widetilde{\xi}^{\beta}(z, X)=\left.\sum_{a \geqslant 0} z^{a} \widetilde{\xi}_{a}^{\beta}(X)\right|_{\mathrm{v}=1}
$$

We assign weights to leaves, edges, and vertices of a labeled graph $\vec{\Gamma} \in \boldsymbol{\Gamma}(\mathcal{Y})$ as follows: 


\section{AlL-GENUS OPEN-CLOSED MIRROR SYMMETRY}

(1) (Open leaves) To each open leaf $l_{j} \in L^{O}(\Gamma)$ with $\alpha\left(l_{j}\right)=\alpha \in G^{*}$ and $k\left(l_{j}\right)=k \in \mathbb{Z}_{\geqslant 0}$, we assign

$$
\left(\mathcal{L}^{\widetilde{\xi}}\right)_{k}^{\alpha}\left(l_{j}\right)=\left[z^{k}\right]\left(\frac{1}{|G| \sqrt{w_{1} w_{2} w_{3}}} \sum_{\beta \in G^{*}} R(-z)_{\alpha}^{\beta} \widetilde{\xi}^{\beta}\left(z, X_{j}\right)\right) .
$$

(2) (Primary leaves) To each primary leaf $l \in L^{o}(\Gamma)$ with $\alpha(l)=\alpha \in G^{*}$ and $k(l)=k \in \mathbb{Z}_{\geqslant 0}$, we assign

$$
\left(\mathcal{L}^{\boldsymbol{\tau}}\right)_{k}^{\alpha}(l)=\left[z^{k}\right]\left(\frac{1}{|G| \sqrt{w_{1} w_{2} w_{3}}} \sum_{\beta \in G^{*}} \sum_{a=1}^{p} \prod_{i=1}^{3} w_{i}^{c_{i}\left(h_{a}\right)} R(-z)_{\alpha}^{\beta} \chi_{\beta}\left(h_{a}\right) \tau_{a}\right) .
$$

By the orthogonality of the characters, we have

$$
\begin{aligned}
& \sum_{\beta \in G^{*}} \sum_{a=1}^{p} \prod_{i=1}^{3} w_{i}^{c_{i}\left(h_{a}\right)} R(-z)_{\alpha}^{\beta} \chi_{\beta}\left(h_{a}\right) \tau_{a} \\
& =\sum_{a=1}^{p} \prod_{i=1}^{3} w_{i}^{c_{i}\left(h_{a}\right)} \chi_{\alpha}\left(h_{a}\right) \tau_{a} \exp \left(\sum_{m=1}^{\infty} \frac{(-1)^{m+1}}{m(m+1)} B_{m+1}\left(c_{i}\left(h_{a}\right)\right)\left(\frac{z}{w_{i}}\right)^{m}\right) .
\end{aligned}
$$

Here we used the facts that $B_{m}(1-x)=(-1)^{m} B_{m}(x)$, that $B_{m}=0$ when $m$ is odd, and that $c_{i}(h)+c_{i}\left(h^{-1}\right)=1-\delta_{c_{i}(h), 0}$.

(3) (Dilaton leaves) To each dilaton leaf $l \in L^{1}(\Gamma)$ with $\alpha(l)=\alpha \in G^{*}$ and $2 \leqslant k(l)=k \in \mathbb{Z}_{\geqslant 0}$, we assign

$$
\left(\mathcal{L}^{1}\right)_{k}^{\alpha}(l)=\left[z^{k-1}\right]\left(-\frac{1}{|G| \sqrt{w_{1} w_{2} w_{3}}} \sum_{\beta \in G^{*}} R(-z)_{\alpha}^{\beta}\right) .
$$

(4) (Edges) To an edge connecting a vertex marked by $\alpha \in G^{*}$ and a vertex marked by $\beta \in G^{*}$, and with heights $k$ and $l$ at the corresponding half-edges, we assign

$$
\mathcal{E}_{k, l}^{\alpha, \beta}(e)=\left[z^{k} w^{l}\right]\left(\frac{1}{z+w}\left(\delta_{\alpha, \beta}-\sum_{\gamma \in G^{*}} R(-z)_{\alpha}^{\gamma} R(-w)_{\beta}^{\gamma}\right)\right) .
$$

(5) (Vertices) To a vertex $v$ with genus $g(v)=g \in \mathbb{Z}_{\geqslant 0}$ and with marking $\alpha(v)=\gamma \in G^{*}$, with $n$ primary or open leaves and half-edges attached to it with heights $k_{1}, \ldots, k_{n} \in \mathbb{Z}_{\geqslant 0}$ and $m$ dilaton leaves with heights $k_{n+1}, \ldots, k_{n+m} \in \mathbb{Z}_{\geqslant 0}$, we assign

$$
\left(|G| \sqrt{w_{1} w_{2} w_{3}}\right)^{2 g-2+\operatorname{val}(v)} \int_{\overline{\mathcal{M}}_{g, n+m}} \psi_{1}^{k_{1}} \cdots \psi_{n+m}^{k_{n+m}} .
$$

Given a labeled graph $\vec{\Gamma} \in \boldsymbol{\Gamma}_{g, \ell, n}(\mathcal{B} G)$ with $L^{O}(\Gamma)=\left\{l_{1}, \ldots, l_{n}\right\}$, we define its A-model weight to be

$$
\begin{aligned}
w_{A}(\vec{\Gamma})= & \prod_{v \in V(\Gamma)}\left(|G| \sqrt{w_{1} w_{2} w_{3}}\right)^{2 g-2+\operatorname{val}(v)}\left\langle\prod_{h \in H(v)} \tau_{k(h)}\right\rangle_{g(v)} \prod_{e \in E(\Gamma)} \mathcal{E}_{k\left(h_{1}(e)\right), k\left(h_{2}(e)\right)}^{\alpha\left(v_{1}(e)\right), \alpha\left(v_{2}(e)\right)}(e) \\
& \cdot \prod_{l \in L^{o}(\Gamma)}\left(\mathcal{L}^{\boldsymbol{\tau}}\right)_{k(l)}^{\alpha(l)}(l) \prod_{j=1}^{n}\left(\mathcal{L}^{\widetilde{\xi}}\right)_{k\left(l_{j}\right)}^{\alpha\left(l_{j}\right)}\left(l_{j}\right) \prod_{l \in L^{1}(\Gamma)}\left(\mathcal{L}^{1}\right)_{k(l)}^{\alpha(l)}(l) .
\end{aligned}
$$


Setting $\hat{\mathbf{u}}=\boldsymbol{\tau}, \hat{\mathbf{u}}_{j}=\widetilde{\xi}\left(X_{j}\right)$, and $\mathbf{w}_{i}=w_{i}$ in [FLZ16, Theorem 4.2], we obtain

$$
\frac{1}{\ell !}\left\langle\boldsymbol{\tau}^{\ell}, \widetilde{\xi}\left(X_{1}\right), \ldots, \widetilde{\xi}\left(X_{n}\right)\right\rangle_{g, \ell+n}^{\mathcal{X}}=\sum_{\vec{\Gamma} \in \boldsymbol{\Gamma}_{g, \ell, n}(\mathcal{B} G)} \frac{w_{A}(\vec{\Gamma})}{|\operatorname{Aut}(\vec{\Gamma})|} .
$$

Let $\boldsymbol{\Gamma}_{g, n}(\mathcal{X}):=\bigcup_{\ell \geqslant 0} \boldsymbol{\Gamma}_{g, \ell, n}(\mathcal{B} G)$. Equations (5.6) and (5.10) and Proposition 5.3 imply the following A-model graph sum formulae.

TheOrem 5.5 (A-model graph sum). We have the following equations:

(i) (Disk invariants)

$$
F_{0,1}^{\mathcal{X},(\mathcal{L}, f)}(\boldsymbol{\tau} ; X)=\Phi_{-2}^{1}(X)+\sum_{a=1}^{p} \tau_{a} \Phi_{-1}^{h_{a}}(X)+\sum_{\vec{\Gamma} \in \boldsymbol{\Gamma}_{0,1}(\mathcal{X})} \frac{w_{A}(\vec{\Gamma})}{|\operatorname{Aut}(\vec{\Gamma})|},
$$

where

$$
\begin{aligned}
& \Phi_{-2}^{1}(X)+\sum_{a=1}^{p} \tau_{a} \Phi_{-1}^{h_{a}}(X) \\
& =\left.\frac{1}{|G|^{2} w_{1} w_{2} w_{3}}\left(\sum_{\gamma \in G^{*}} \widetilde{\xi}_{-2}^{\gamma}(X)+\sum_{a=1}^{p} \tau_{a} \prod_{i=1}^{3} w_{i}^{c_{i}\left(h_{a}\right)} \sum_{\gamma \in G^{*}} \chi_{\gamma}\left(h_{a}\right) \widetilde{\xi}_{-1}^{\gamma}(X)\right)\right|_{v=1} .
\end{aligned}
$$

(ii) (Annulus invariants)

$$
F_{0,2}^{\mathcal{X},(\mathcal{L}, f)}\left(\boldsymbol{\tau} ; X_{1}, X_{2}\right)=F_{0,2}^{\mathcal{X},(\mathcal{L}, f)}\left(0 ; X_{1}, X_{2}\right)+\sum_{\vec{\Gamma} \in \boldsymbol{\Gamma}_{0,2}(\mathcal{X})} \frac{w_{A}(\vec{\Gamma})}{|\operatorname{Aut}(\vec{\Gamma})|},
$$

where $F_{0,2}^{\mathcal{X},(\mathcal{L}, f)}\left(0 ; X_{1}, X_{2}\right)$ is determined by equation (5.5).

(iii) For $2 g-2+n>0$,

$$
F_{g, n}^{\mathcal{X},(\mathcal{L}, f)}\left(\boldsymbol{\tau} ; X_{1}, \ldots, X_{n}\right)=\sum_{\vec{\Gamma} \in \boldsymbol{\Gamma}_{g, n}(\mathcal{X})} \frac{w_{A}(\vec{\Gamma})}{|\operatorname{Aut}(\vec{\Gamma})|} .
$$

\section{The mirror curve and its compactification}

The equation of the framed mirror curve is given by

$$
X^{r} Y^{-s-r f}+Y^{m}+1+\sum_{a=1}^{p} q_{a} X^{m_{a}} Y^{n_{a}-m_{a} f}=0
$$

where

$$
m_{a}=r c_{1}\left(h_{a}\right), \quad n_{a}=-s c_{1}\left(h_{a}\right)+m c_{2}\left(h_{a}\right) .
$$

Let $\triangle$ be the triangle on $\mathbb{R}^{2}$ with vertices $(r,-s),(0, m)$, and $(0,0)$. The compactified mirror curve $\bar{\Sigma}_{q}$ is embedded in the toric surface $S_{\triangle}$ defined by the polytope $\triangle$. In this section, we assume that $q_{1}, \ldots, q_{p} \in \mathbb{C}$ are sufficiently small so that the compactified mirror curve $\bar{\Sigma}_{q}$ is a smooth compact Riemann surface. Let $\mathfrak{g}$ be the genus of $\bar{\Sigma}_{q}$, and let $\mathfrak{n}$ be the number of points in $\bar{\Sigma}_{q} \backslash \Sigma_{q}$. 


\section{All-Genus OPEN-ClOSED MIRROR SYMMETRY}

\subsection{The Liouville 1-form}

Let $\widetilde{\Sigma}_{q}:=\pi^{-1}\left(\Sigma_{q}\right) \subset \mathbb{C}^{2}$ be the preimage of $\Sigma_{q} \subset\left(\mathbb{C}^{*}\right)^{2}$ under the (universal) covering map $\pi: \mathbb{C}^{2} \rightarrow\left(\mathbb{C}^{*}\right)^{2}$ defined by $(x, y) \mapsto\left(X=e^{-x}, Y=e^{-y}\right)$. Then $\pi_{q}:=\left.\pi\right|_{\widetilde{\Sigma}_{q}}: \widetilde{\Sigma}_{q} \rightarrow \Sigma_{q}$ is a regular $\mathbb{Z}^{2}$-cover. The 1 -form $\log Y d X / X$ is multi-valued on $\left(\mathbb{C}^{*}\right)^{2}$, but $\pi^{*}(\log Y d X / X)=y d x$ is a well-defined holomorphic 1-form on $\mathbb{C}^{2}$; indeed, it is the Liouville 1-form of the cotangent bundle $T^{*} \mathbb{C}=(\mathbb{C})^{2}$ :

$$
d(y d x)=d y \wedge d x
$$

We define

$$
\Phi_{q}:=\left.\left(\log Y \frac{d X}{X}\right)\right|_{\Sigma_{q}},
$$

which is a multi-valued 1-form on $\Sigma_{q}$. Then $\pi_{q}^{*} \Phi_{q}=\left.y d x\right|_{\widetilde{\Sigma}_{q}}$ is a well-defined holomorphic 1-form on $\widetilde{\Sigma}_{q}$.

Given a $q \in \mathbb{C}^{p}$ such that $\Sigma_{q}$ is smooth, we choose a base point $p_{q} \in \Sigma_{q}$ and a point $\tilde{p}_{q} \in \pi_{q}^{-1}\left(p_{q}\right)$. We have a commutative diagram

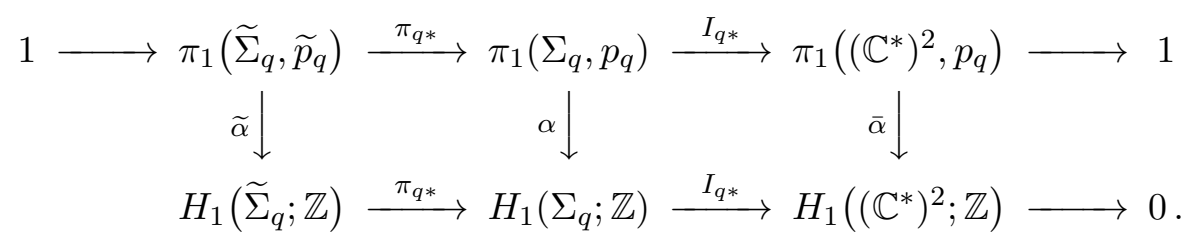

In the above diagram, the following hold:

- The map $I_{q^{*}}$ is induced by the inclusion map $I_{q}: \Sigma_{q} \hookrightarrow\left(\mathbb{C}^{*}\right)^{2}$.

- The first row is a short exact sequence of multiplicative groups.

- The second row is exact at $H_{1}\left(\Sigma_{q} ; \mathbb{Z}\right)=\mathbb{Z}^{2 \mathfrak{g}+\mathfrak{n}-1}$ and $H_{1}\left(\left(\mathbb{C}^{*}\right)^{2} ; \mathbb{Z}\right)=\mathbb{Z}^{2}$.

- The maps $\widetilde{\alpha}$ and $\alpha$ are surjective group homomorphisms given by abelianization, and $\bar{\alpha}$ is a group isomorphism.

- The group $\pi_{1}\left(\Sigma_{q}, p_{q}\right)$ is a free group generated by $2 \mathfrak{g}+\mathfrak{n}-1$ elements.

We define

$$
K_{1}\left(\Sigma_{q} ; \mathbb{Z}\right):=\operatorname{Ker}\left(I_{q^{*}}: H_{1}\left(\Sigma_{q} ; \mathbb{Z}\right) \rightarrow H_{1}\left(\left(\mathbb{C}^{*}\right)^{2} ; \mathbb{Z}\right)\right)=\operatorname{Im}\left(\pi_{q^{*}}: H_{1}\left(\widetilde{\Sigma_{q}} ; \mathbb{Z}\right) \rightarrow H_{1}\left(\Sigma_{q} ; \mathbb{Z}\right)\right) .
$$

Then $K_{1}\left(\Sigma_{q} ; \mathbb{Z}\right) \cong \mathbb{Z}^{2 \mathfrak{g}+\mathfrak{n}-3}$. There is a well-defined map

$$
\pi_{1}\left(\widetilde{\Sigma}_{q}, \widetilde{p}_{q}\right) \rightarrow \mathbb{C}, \quad \widetilde{A} \mapsto \int_{\widetilde{A}} \pi_{q}^{*} \Phi_{q}
$$

Given any $A \in K_{1}\left(\Sigma_{q} ; \mathbb{Z}\right)$, there exists an $\widetilde{A} \in \pi_{1}\left(\widetilde{\Sigma}_{q}, \widetilde{p}_{q}\right)$ such that $A=\pi_{q *} \circ \widetilde{\alpha}(\widetilde{A})=\alpha \circ \pi_{q *}(\widetilde{A})$. If $\widetilde{A}_{1}, \widetilde{A}_{2} \in \pi_{1}\left(\widetilde{\Sigma}_{q}, \widetilde{p}_{q}\right)$ and $\alpha \circ \pi_{q *}\left(\widetilde{A}_{1}\right)=\alpha \circ \pi_{q *}\left(\widetilde{A}_{2}\right)=A$, then $\pi_{q *}\left(\widetilde{A}_{2}\right)=\pi_{q *}\left(\widetilde{A}_{1}\right) B_{1} B_{2} B_{1}^{-1} B_{2}^{-1}$ for some $B_{1}, B_{2} \in \pi_{1}\left(\Sigma_{q}, p_{q}\right)$. If $I_{*}\left(B_{1}\right)=\left(m_{1}, n_{1}\right)$ and $I_{*}\left(B_{2}\right)=\left(m_{2}, n_{2}\right)$, where $m_{1}, n_{1}, m_{2}, n_{2} \in \mathbb{Z}$, then

So we have a map

$$
\int_{\widetilde{A}_{2}} \pi_{q}^{*} \Phi=\int_{\widetilde{A}_{1}} \pi_{q}^{*} \Phi+(2 \pi \sqrt{-1})^{2}\left(m_{1} n_{2}-m_{2} n_{1}\right) .
$$

$$
K_{1}\left(\Sigma_{q} ; \mathbb{Z}\right) \rightarrow \mathbb{C} /(2 \pi \sqrt{-1})^{2} \mathbb{Z}, \quad A \mapsto \int_{\widetilde{A}} \pi_{q}^{*} \Phi_{q}+(2 \pi \sqrt{-1})^{2} \mathbb{Z},
$$


where $\widetilde{A}$ is any element in $\left(\pi_{q *} \circ \widetilde{\alpha}\right)^{-1}(A) \subset \pi_{1}\left(\widetilde{\Sigma}_{q}, \widetilde{p}_{q}\right)$. Let $\int_{A} \Phi$ denote the image of $A \in$ $K_{1}\left(\Sigma_{q} ; \mathbb{Z}\right)$ under the above map. Then $\int_{A} \Phi_{q} \in \mathbb{C} /(2 \pi \sqrt{-1})^{2} \mathbb{Z}$ is independent of choice of $p_{q}$ and $\widetilde{p}_{q}$.

Let $B_{\epsilon}$ denote the open ball in $\mathbb{C}^{p}$ with radius $\epsilon>0$ and center 0 . There exists an $\epsilon>0$ such that $\Sigma_{q}$ is smooth for all $q \in B_{\epsilon}$. For $q \in B_{\epsilon}$, there are canonical isomorphisms

$$
H_{1}\left(\Sigma_{q} ; \mathbb{Z}\right) \cong H_{1}\left(\Sigma_{0} ; \mathbb{Z}\right), \quad K_{1}\left(\Sigma_{q} ; \mathbb{Z}\right) \cong K_{1}\left(\Sigma_{0} ; \mathbb{Z}\right)
$$

Given an $A \in K_{1}\left(\Sigma_{0} ; \mathbb{Z}\right) \cong K_{1}\left(\Sigma_{q} ; \mathbb{Z}\right)$, the integral $\int_{A} \Phi_{q}$ can be viewed as an element in $\Gamma\left(B_{\epsilon}, \mathbb{C}\right) /(2 \pi \sqrt{-1})^{2} \mathbb{Z}$, where $\Gamma\left(B_{\epsilon}, \mathbb{C}\right)$ is the space of holomorphic functions on $B_{\epsilon}$ and $(2 \pi \sqrt{-1})^{2} \mathbb{Z}$ is identified with the set of constant functions from $B_{\epsilon}$ to $(2 \pi \sqrt{-1})^{2} \mathbb{Z}$.

The inclusion $J_{q}: \Sigma_{q} \hookrightarrow \bar{\Sigma}_{q}$ induces a surjective map

$$
J_{q^{*}}: H_{1}\left(\Sigma_{q} ; \mathbb{C}\right) \cong \mathbb{C}^{2 \mathfrak{g}+\mathfrak{n}-1} \rightarrow H_{1}\left(\bar{\Sigma}_{q} ; \mathbb{C}\right) \cong \mathbb{C}^{2 \mathfrak{g}},
$$

which restricts to a surjective $\mathbb{C}$-linear map

$$
K_{1}\left(\Sigma_{q} ; \mathbb{C}\right):=K_{1}\left(\Sigma_{q} ; \mathbb{Z}\right) \otimes_{\mathbb{Z}} \mathbb{C} \cong \mathbb{C}^{2 \mathfrak{g}+\mathfrak{n}-3} \rightarrow H_{1}\left(\bar{\Sigma}_{q} ; \mathbb{C}\right) \cong \mathbb{C}^{2 \mathfrak{g}} .
$$

The complex vector spaces $K_{1}\left(\Sigma_{q} ; \mathbb{C}\right), H_{1}\left(\Sigma_{q} ; \mathbb{C}\right)$, and $H_{1}\left(\bar{\Sigma}_{q} ; \mathbb{C}\right)$ form flat complex vector bundles $\mathcal{K}, \mathcal{H}$, and $\overline{\mathcal{H}}$ over $B_{\epsilon}$ of rank $2 \mathfrak{g}+\mathfrak{n}-3,2 \mathfrak{g}+\mathfrak{n}-1$, and $2 \mathfrak{g}$, respectively. Given $A \in K_{1}\left(\Sigma_{q} ; \mathbb{C}\right)$ which is a flat section of $\mathcal{K}$ with respect to the Gauss-Manin connection, $\int_{A} \Phi_{q}$ can be viewed as an element in $\Gamma\left(B_{\epsilon}, \mathbb{C}\right) / \mathbb{C}$, where $\mathbb{C}$ is identified with the space of constant functions from $B_{\epsilon}$ to $\mathbb{C}$.

\subsection{Holomorphic 1-forms on $\boldsymbol{\Sigma}_{q}$}

For any integers $m, n$,

$$
\varpi_{m, n}:=\operatorname{Res}_{H_{f}=0} \frac{X^{m} Y^{n-f m}}{H_{f}} \cdot \frac{d X}{X} \wedge \frac{d Y}{Y}=\frac{-X^{m} Y^{n-f m}}{Y \partial H_{f}(X, Y, q) / \partial Y} \frac{d X}{X}
$$

is a holomorphic 1-form on $\Sigma_{q}$. We view $X$ as a flat coordinate independent of $q$ and use implicit differentiation to obtain

$$
\frac{\partial Y}{\partial q_{a}}=\frac{-X^{m_{a}} Y^{n_{a}-f m_{a}}}{\partial H / \partial Y}
$$

We define

Then $\nabla_{\partial / \partial q_{a}} \Phi=\varpi_{m_{a}, n_{a}}$ for $\mathrm{a}=1, \ldots, \mathrm{p}$.

$$
\nabla_{\partial / \partial q_{a}} \Phi:=\left.\frac{\partial \Phi}{\partial q_{a}}\right|_{X=\text { constant }}=\frac{\partial Y}{\partial q_{a}} \frac{d X}{Y X} .
$$

\subsection{Differentials of the first kind on $\bar{\Sigma}_{q}$}

Recall that a differential of the first kind is a holomorphic 1-form. By results in [BC94], the holomorphic 1-form $\varpi_{m, n}$ on $\Sigma_{q}$ extends to a holomorphic 1-form on $\bar{\Sigma}_{q}$ if and only if $(m, n)$ is in the interior of the triangle $\triangle$ with vertices $(r,-s),(0, m),(0,0)$. Without loss of generality, we may assume that $\left(m_{a}, n_{a}\right)$ is in the interior of $\triangle$ for $1 \leqslant a \leqslant \mathfrak{g}$ and is on the boundary of $\triangle$ for $\mathfrak{g}+1 \leqslant a \leqslant p$; note that $\mathfrak{g}$ can be zero. Let $\mathfrak{n}=p-\mathfrak{g}+3$ be the number of lattice points on the boundary of $\triangle$. Then $\Sigma_{q}$ is a Riemann surface of genus $\mathfrak{g}$ with $\mathfrak{n}$ punctures, and $\bar{\Sigma}_{q}$ is a compact Riemann surface of genus $\mathfrak{g}$. We have

$$
p=\operatorname{dim}_{\mathbb{C}} H_{\mathrm{CR}}^{2}(\mathcal{X} ; \mathbb{C}), \quad \mathfrak{g}=\operatorname{dim}_{\mathbb{C}} H_{\mathrm{CR}}^{4}(\mathcal{X} ; \mathbb{C}),
$$




\section{AlL-GENUS OPEN-CLOSED MIRROR SYMMETRY}

and

$$
-\chi\left(\Sigma_{q}\right)=2 \mathfrak{g}-2+\mathfrak{n}=1+p+\mathfrak{g}=|G|=\operatorname{dim}_{\mathbb{C}} H_{\mathrm{CR}}^{*}(\mathcal{X} ; \mathbb{C}) .
$$

A basis of $H^{0}\left(\bar{\Sigma}_{q}, \omega_{\bar{\Sigma}_{q}}\right)=H^{1,0}\left(\bar{\Sigma}_{q}, \mathbb{C}\right)$ is given by $\left\{\nabla_{\partial / \partial q_{a}} \Phi=\varpi_{m_{a}, n_{a}}: a=1, \ldots, \mathfrak{g}\right\}$.

Let $B_{\epsilon}=\left\{\left(q_{1}, \ldots, q_{p}\right) \in \mathbb{C}^{p}:|q|<\epsilon\right\}$ be an open ball in $\mathbb{C}^{p}$ with radius $\epsilon>0$ centered at the origin. Let $\boldsymbol{\Sigma}_{\epsilon}=\left\{(X, Y, q) \in \mathbb{C}^{*} \times \mathbb{C}^{*} \times \mathbb{C}^{p}: H_{f}(X, Y, q)=0\right\}$ be the family of mirror curves over $B_{\epsilon}$, and let $\pi_{\epsilon}: \boldsymbol{\Sigma}_{\epsilon} \rightarrow B_{\epsilon}$ be given by $(X, Y, q) \mapsto q$, so that $\pi_{\epsilon}^{-1}(q)=\Sigma_{q}$. Then $G^{*}$ acts on the total space $\boldsymbol{\Sigma}_{\epsilon}$ by

$$
\alpha \cdot\left(X, Y, q_{a}\right)=\left(\chi_{\alpha}\left(\eta_{1}\right) X, \chi_{\alpha}\left(\eta_{2}\right) Y, \chi_{\alpha}\left(h_{a}^{-1}\right) q_{a}\right),
$$

where $\alpha \in G^{*}, \chi_{\alpha}: G \rightarrow \mathbb{C}^{*}$ is the associated character, $\eta_{1}, \eta_{2} \in G$ are defined as in equation (2.6), and $h_{a} \in G$ corresponds to $\left(m_{a}, n_{a}, 1\right) \in \operatorname{Box}(\sigma)$ for $a=1, \ldots, p$. The $G^{*}$-action on $\boldsymbol{\Sigma}_{\epsilon}$ extends to the family of compactified mirror curve $\bar{\pi}_{\epsilon}: \bar{\Sigma}_{\epsilon} \rightarrow B_{\epsilon}$, where $\bar{\pi}_{\epsilon}^{-1}(q)=\bar{\Sigma}_{q}$. In particular, $G^{*}$ acts on $\bar{\Sigma}_{0}$, and the induced $G^{*}$-action on $H_{1}\left(\bar{\Sigma}_{0} ; \mathbb{C}\right)$ preserves the intersection pairing $\cdot$ on $H_{1}\left(\bar{\Sigma}_{0} ; \mathbb{C}\right)$. We choose a symplectic basis $\bar{A}_{1}, \bar{B}_{1}, \ldots, \bar{A}_{\mathfrak{g}}, \bar{B}_{\mathfrak{g}}$ of $H_{1}\left(\bar{\Sigma}_{0} ; \mathbb{C}\right)$ such that

$$
\bar{A}_{a} \cdot \bar{A}_{b}=\bar{B}_{a} \cdot \bar{B}_{b}=0, \quad \bar{A}_{a} \cdot \bar{B}_{b}=\delta_{a b}, \quad a, b=1, \ldots, \mathfrak{g}
$$

and

$$
\left.\frac{1}{2 \pi \sqrt{-1}} \int_{\bar{A}_{a}}\left(\nabla_{\partial / \partial q_{b}} \Phi\right)\right|_{q=0}=\delta_{a b}, \quad a, b=1, \ldots, \mathfrak{g} .
$$

The $G^{*}$-action on $\bar{A}_{a}$ is given by $\alpha \cdot \bar{A}_{a}=\chi_{\alpha}\left(h_{a}\right) \bar{A}_{a}$, where $\alpha \in G^{*}$ and $1 \leqslant a \leqslant \mathfrak{g}$. We may choose $\bar{B}_{a}$ such that $\alpha \cdot \bar{B}_{a}=\chi_{\alpha}\left(h_{a}^{-1}\right) \bar{B}_{a}$ for $\alpha \in G^{*}$ and $1 \leqslant a \leqslant \mathfrak{g}$.

The inclusion $I_{0}: \Sigma_{0} \rightarrow\left(\mathbb{C}^{*}\right)^{2}$ is $G^{*}$-equivariant, where $\alpha \in G^{*}$ acts on $\left(\mathbb{C}^{*}\right)^{2}$ by $\alpha \cdot(X, Y)=$ $\left(\chi_{\alpha}\left(\eta_{1}\right) X, \chi_{\alpha}\left(\eta_{2}\right) Y\right)$. So the $G^{*}$-actions on $H_{1}\left(\left(\mathbb{C}^{*}\right)^{2} ; \mathbb{Z}\right)$ and $H_{1}\left(\left(\mathbb{C}^{*}\right)^{2} ; \mathbb{C}\right)$ are trivial, and the $G^{*}$-action on $H_{1}\left(\Sigma_{0} ; \mathbb{C}\right)$ preserves $K_{1}\left(\Sigma_{0} ; \mathbb{C}\right)=\operatorname{Ker}\left(I_{*}: H_{1}\left(\Sigma_{0} ; \mathbb{C}\right) \rightarrow H_{1}\left(\left(\mathbb{C}^{*}\right)^{2} ; \mathbb{Z}\right)\right.$.

We extend $\bar{A}_{1}, \bar{B}_{1}, \ldots, \bar{A}_{\mathfrak{g}}, \bar{B}_{\mathfrak{g}}$ to a symplectic basis of $H_{1}\left(\bar{\Sigma}_{q} ; \mathbb{C}\right)$ such that $\bar{A}_{i}$ and $\bar{B}_{i}$ are flat sections of $\overline{\mathcal{H}}$ with respect to the Gauss-Manin connection. Note that the composition

$$
K_{1}\left(\Sigma_{q} ; \mathbb{C}\right) \rightarrow H_{1}\left(\Sigma_{q} ; \mathbb{C}\right) \stackrel{J_{*}}{\longrightarrow} H_{1}\left(\bar{\Sigma}_{q} ; \mathbb{C}\right)
$$

is surjective. For $a=1, \ldots, \mathfrak{g}$, we lift $\bar{A}_{a}, \bar{B}_{a} \in H_{1}\left(\bar{\Sigma}_{q} ; \mathbb{C}\right)$ to $A_{a}, B_{a} \in K_{1}\left(\Sigma_{q} ; \mathbb{C}\right)$. We choose $A_{a}, B_{a} \in K_{1}\left(\Sigma_{q} ; \mathbb{C}\right)$ such that they are flat sections of $\mathcal{K}$ with respect to the Gauss-Manin connection and are eigenvectors of the $G^{*}$-action on $K_{1}\left(\Sigma_{0} ; \mathbb{C}\right)$ at $q=0$.

\subsection{Differentials of the third kind on $\overline{\boldsymbol{\Sigma}}_{q}$}

Recall that a differential of the third kind is a meromorphic 1-form with only simple poles.

Let $E_{1}, E_{2}, E_{3}$ be the edges of the triangle $\triangle$ opposite to the vertices $(r,-s),(0, m),(0,0)$, respectively. Let $\mathfrak{n}_{i}+1$ be the number of lattice points on $E_{i}$ (including the end points). Then $G_{i}:=\left\{h \in G: \chi_{i}(h)=1\right\} \cong \boldsymbol{\mu}_{\mathfrak{n}_{i}} ;$ in particular, $\mathfrak{n}_{1}=m$. For $i=1,2,3$, we have short exact sequences of abelian groups

$$
1 \rightarrow G_{i}=\boldsymbol{\mu}_{\mathfrak{n}_{i}} \rightarrow G \stackrel{\chi_{i}}{\rightarrow} \boldsymbol{\mu}_{r_{i}} \rightarrow 1, \quad 1 \rightarrow \boldsymbol{\mu}_{r_{i}}^{*} \rightarrow G^{*} \rightarrow G_{i}^{*} \rightarrow 1 .
$$

The $G^{*}$-action on $\bar{\Sigma}_{0}$ preserves the finite set $\bar{\Sigma}_{0} \cap D_{i}$, where $D_{i} \subset S_{\triangle}$ is the torus-invariant divisor associated with the edge $E_{i}$. The $G^{*}$-action on $\bar{\Sigma}_{0} \cap D_{i}$ induces a free and transitive $G_{i}^{*}$-action on $\bar{\Sigma}_{0} \cap D_{i}$. So we may label the point in $\bar{\Sigma}_{0} \cap D_{i}$ (non-canonically) by elements in $G_{i}^{*}=\boldsymbol{\mu}_{\mathfrak{n}_{i}}^{*}$; that is, $\bar{\Sigma}_{0} \cap D_{i}=\left\{\bar{p}_{\ell}^{i}: \ell \in \boldsymbol{\mu}_{\mathfrak{n}_{i}}^{*}\right\}$.

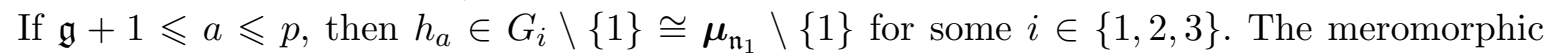
1 -form $\nabla_{\partial / \partial q_{a}} \Phi$ has a simple pole at each point in $\left\{\bar{p}_{\ell}^{i}: \ell \in G_{i}^{*}\right\}$ and is holomorphic elsewhere 


\section{B. FAnG, C.-C. M. LiU And Z. Zong}

on the compactified mirror curve $\bar{\Sigma}_{q}$, so it is a differential of the third kind on $\bar{\Sigma}_{q}$. We have

$$
\left.\operatorname{Res}_{p \rightarrow \bar{p}_{\ell}^{i}}\left(\nabla_{\partial / \partial q_{a}} \Phi\right)\right|_{q=0}=\frac{e^{\pi \sqrt{-1} k / \mathfrak{n}_{i}}}{\mathfrak{n}_{i}} \chi_{\ell}(k),
$$

where $h_{a}=e^{2 \pi \sqrt{-1} k / \mathfrak{n}_{i}} \in \boldsymbol{\mu}_{\mathfrak{n}_{i}}$ for $1 \leqslant k \leqslant \mathfrak{n}_{i}-1$ and $\chi_{\ell}: G_{i} \rightarrow \mathbb{C}^{*}$ is the character associated with $\ell \in G_{i}^{*}$. Let $C_{\ell}^{i} \in H_{1}\left(\Sigma_{0} ; \mathbb{Z}\right)$ be the class of a small circle around $\bar{p}_{\ell}^{i}$. Then there exists an $A_{a} \in K_{1}\left(\Sigma_{0} ; \mathbb{C}\right)(a=\mathfrak{g}+1, \ldots, p)$ such that

- $A_{a}$ is an eigenvector of the $G^{*}$-action on $K_{1}\left(\Sigma_{0} ; \mathbb{C}\right)$;

- $A_{a}$ is a $\mathbb{C}$-linear combination of $C_{\ell}^{i}$, with $\ell \in G_{i}^{*}$;

- for $a, b=1, \ldots, p$,

$$
\left.\frac{1}{2 \pi \sqrt{-1}} \int_{A_{a}}\left(\nabla_{\partial / \partial q_{b}} \Phi\right)\right|_{q=0}=\delta_{a b} .
$$

We extend $A_{\mathfrak{g}+1}, \ldots, A_{p}$ to flat sections of $K_{1}\left(\Sigma_{q} ; \mathbb{C}\right)$. Then for $a, b \in\{1, \ldots, p\}$,

$$
\frac{1}{2 \pi \sqrt{-1}} \int_{A_{a}} \nabla_{\partial / \partial q_{b}} \Phi=\delta_{a b}+O(|q|) .
$$

Definition 6.1 (B-model closed string flat coordinates). For $a=1, \ldots, p$, let $\tau_{a}(q)$ be the unique function in $\Gamma\left(B_{\epsilon}, \mathbb{C}\right)$ such that $\tau_{a}(0)=0$ and

$$
\frac{1}{2 \pi \sqrt{-1}} \int_{A_{a}} \Phi=\tau_{a}(q)+\mathbb{C} \in \Gamma\left(B_{\epsilon}, \mathbb{C}\right) / \mathbb{C} .
$$

Then $\tau_{a}(q)=q_{a}+O\left(|q|^{2}\right)$ and

$$
\frac{1}{2 \pi \sqrt{-1}} \int_{A_{a}} \nabla_{\partial / \partial \tau_{b}} \Phi=\delta_{a b}, \quad a, b=1, \ldots, p .
$$

The B-model closed string flat coordinates are related to $q_{a}$ for $a=1, \ldots, p$ via the following hypergeometric formulae:

$$
\tau_{a}(q)=q_{a}\left(\sum_{\left\{\sum_{b=1}^{p} d_{b} c_{i}\left(h_{b}\right)\right\}=0, i=1,2,3} \prod_{i=1}^{3} \frac{\Gamma\left(-\left\{c_{i}\left(h_{a}\right)\right\}+1\right)}{\Gamma\left(-c_{i}\left(h_{a}\right)-\sum_{b=1}^{p} d_{b} c_{i}\left(h_{b}\right)+1\right)} \cdot \frac{1}{\prod_{b=1}^{p} d_{b} !} \prod_{b=1}^{p} q_{b}^{d_{b}}\right) .
$$

Here $\{x\}$ denotes the fractional part of $x$. This mirror map is obtained by solving the GelfandKapranov-Zelevinsky-type (GKZ-type for short) Picard-Fuchs equations. Iritani explains these GKZ-operators and explicitly writes down the mirror map for general toric orbifolds [Iri09]. The integrals over A-cycles on mirror curves as flat coordinates and their hypergeometric expressions for toric Calabi-Yau 3-folds are discussed in [CKYZ99] together with genus zero closed GromovWitten mirror symmetry.

Let $D_{q}^{\infty}=\bar{\Sigma}_{q} \backslash \Sigma_{q}=\bigcup_{i=1}^{3}\left\{\bar{p}_{1}^{i}, \ldots, \bar{p}_{\mathfrak{n}_{i}}^{i}\right\}$. By Lefschetz duality, there is a perfect pairing

$$
H_{1}\left(\Sigma_{q} ; \mathbb{C}\right) \times H_{1}\left(\bar{\Sigma}_{q}, D_{q}^{\infty} ; \mathbb{C}\right) \rightarrow \mathbb{C} .
$$

The inclusion $\Sigma_{q} \subset \bar{\Sigma}_{q}$ induces a surjective $\mathbb{C}$-linear map

$$
H_{1}\left(\Sigma_{q} ; \mathbb{C}\right) \cong \mathbb{C}^{2 \mathfrak{g}+\mathfrak{n}-1} \rightarrow H_{1}\left(\bar{\Sigma}_{q} ; \mathbb{Z}\right) \cong \mathbb{C}^{2 g} .
$$

Our choice of $A_{1}, B_{1}, \ldots, A_{\mathfrak{g}}, B_{\mathfrak{g}}$ gives a splitting

$$
j_{1}: H_{1}\left(\bar{\Sigma}_{q} ; \mathbb{C}\right) \rightarrow H_{1}\left(\Sigma_{q} ; \mathbb{C}\right) .
$$




\section{All-Genus open-ClOSED MIRROR SYMMETRY}

The long exact sequence of relative homology groups of the pair $\left(\bar{\Sigma}_{q}, D_{q}^{\infty}\right)$ gives an injective $\mathbb{C}$-linear map

$$
j_{2}: H_{1}\left(\bar{\Sigma}_{q} ; \mathbb{C}\right) \rightarrow H_{1}\left(\bar{\Sigma}_{q}, D_{q}^{\infty} ; \mathbb{C}\right) .
$$

Under the inclusion maps $j_{1}$ and $j_{2}$, the perfect pairing (6.6) restricts to the intersection pairing

$$
H_{1}\left(\bar{\Sigma}_{q} ; \mathbb{C}\right) \times H_{1}\left(\bar{\Sigma}_{q} ; \mathbb{C}\right) \rightarrow \mathbb{C},
$$

which is perfect by Poincaré duality. For $\mathfrak{g}+1 \leqslant a \leqslant p$, choose $B_{a} \in H_{1}\left(\bar{\Sigma}_{q}, D_{q}^{\infty} ; \mathbb{C}\right)$ such that

- $\left(A_{1}, \ldots, A_{p}, B_{1}, \ldots, B_{\mathfrak{g}}\right)$ and $\left(B_{1}, \ldots, B_{p},-A_{1}, \ldots,-A_{\mathfrak{g}}\right)$ are dual under the pairing (6.6);

- $\alpha \cdot B_{a}=\chi_{\alpha}\left(h_{a}^{-1}\right) B_{a}$ for $\alpha \in G^{*}$, and $\mathfrak{g}+1 \leqslant a \leqslant p$.

Then for $a=1, \ldots, p$,

$$
\left(\nabla_{\partial / \partial \tau_{a}} \Phi\right)(z)=\int_{z^{\prime} \in B_{a}} B\left(z^{\prime}, z\right)
$$

where $B\left(z^{\prime}, z\right)$ is the fundamental normalized differential of the second kind on $\bar{\Sigma}_{q}$ (see Section 6.6).

\subsection{Critical points and Lefschetz thimbles}

We choose a framing $f$ such that $X: \Sigma_{0} \rightarrow \mathbb{C}^{*}$ has only simple branch points. Then $X: \Sigma_{q} \rightarrow \mathbb{C}^{*}$ has only simple branch points for $q$ sufficiently small.

The critical points of $X: \Sigma_{0}=\left\{(X, Y) \in\left(\mathbb{C}^{*}\right)^{2}: X^{r} Y^{-s-r f}+Y^{m}+1=0\right\} \rightarrow \mathbb{C}^{*}$ are

$$
\left\{\left(X_{j \ell}, Y_{j \ell}\right): j \in\{0,1, \ldots, r-1\}, \ell \in\{0,1, \ldots, m-1\}\right\},
$$

where

$$
\begin{aligned}
X_{j \ell} & =\exp \left(2 \pi \sqrt{-1}\left(\frac{j}{r}+\ell \frac{s+r f}{r m}\right)\right) m^{1 / r}(s+r f)^{(s+r f) / r m}(-m-s-r f)^{(-m-s-r f) / r m} \\
& =\left(\chi_{1}^{j} \chi_{2}^{\ell}\right)\left(\eta_{1}\right) \prod_{i=1}^{3}\left(|G| w_{i}\right)^{w_{i}} \\
Y_{j \ell} & =\exp \left(2 \pi \sqrt{-1} \frac{\ell}{m}\right)\left(\frac{s+r f}{-m-s-r f}\right)^{1 / m}=\left(\chi_{1}^{j} \chi_{2}^{\ell}\right)\left(\eta_{2}\right)\left(\frac{w_{2}}{w_{3}}\right)^{1 / m} .
\end{aligned}
$$

If $\alpha=\chi_{1}^{j} \chi_{2}^{\ell} \in G^{*}$, then we define $\left(X_{\alpha}, Y_{\alpha}\right)=\left(X_{j \ell}, Y_{j \ell}\right)$. The critical points of $X: \Sigma_{0} \rightarrow \mathbb{C}^{*}$ are $\left\{\left(X_{\alpha}, Y_{\alpha}\right): \alpha \in G^{*}\right\}$. We define

$$
\begin{aligned}
a_{\alpha} & :=-\log \left(X_{\alpha}\right)=-\sum_{i=1}^{3} w_{i} \log w_{i}-\log \left(\chi_{\alpha}\left(\eta_{1}\right)\right), \\
b_{\alpha} & :=-\log \left(Y_{\alpha}\right)=\frac{1}{m} \log \left(w_{3} / w_{2}\right)-\log \left(\chi_{\alpha}\left(\eta_{2}\right)\right) .
\end{aligned}
$$

In equation (6.9), we use the identity $\sum_{i=1}^{3} w_{i} \log \left(|G| w_{i}\right)=\sum_{i=1}^{3} w_{i} \log w_{i}$, which holds since $\sum_{i=1}^{3} w_{i}=0$. Note that

$$
\log \left(\chi_{\alpha}\left(\eta_{1}\right)\right)=\sqrt{-1} \vartheta_{\alpha}, \quad \log \left(\chi_{\alpha}\left(\eta_{2}\right)\right)=\sqrt{-1} \varphi_{\alpha}
$$

for some $\vartheta_{\alpha}, \varphi_{\alpha} \in \mathbb{R}$. We may assume $\vartheta_{\alpha}, \varphi_{\alpha} \in[0,2 \pi)$.

Let $X=e^{-x}$ and $Y=e^{-y}$. Around each critical point $p_{\alpha}$, we set up the following local 
coordinates

$$
x=a_{\alpha}(q)+\zeta_{\alpha}(q)^{2}, \quad y=b_{\alpha}(q)+\sum_{d=1}^{\infty} h_{d}^{\alpha}(q) \zeta_{\alpha}(q)^{d},
$$

where

$$
h_{1}^{\alpha}(q)=\sqrt{\frac{2}{\left(d^{2} x / d y^{2}\right)\left(b_{\alpha}(q)\right)}} .
$$

Let $\gamma_{\alpha}$ be the Lefschetz thimble of the superpotential $x=-\log X: \Sigma_{q} \rightarrow \mathbb{C}$, so that $x\left(\gamma_{\alpha}\right)=$ $a_{\alpha}+\mathbb{R}^{+}$. Then $\left\{\gamma_{\alpha}: \alpha \in G^{*}\right\}$ is a basis of the relative homology group

$$
H_{1}\left(\Sigma_{q},\left\{(X, Y) \in \Sigma_{q}: \log X \ll 0\right\} ; \mathbb{Z}\right) \cong \mathbb{Z}^{|G|} .
$$

\subsection{Differentials of the second kind on $\overline{\boldsymbol{\Sigma}}_{q}$}

Recall that a differential of the second kind is a meromorphic 1-form with no residues.

We choose a symplectic basis $\left\{A_{1}, B_{1}, \ldots, A_{\mathfrak{g}}, B_{\mathfrak{g}}\right\}$ of $H_{1}\left(\bar{\Sigma}_{q}, \mathbb{C}\right)$ as in Section 6.3. Let $B\left(p_{1}, p_{2}\right)$ be the fundamental normalized differential of the second kind on $\bar{\Sigma}_{q}$ (see, for example, [Fay73]), which is characterized by the following properties:

(1) The differential $B\left(p_{1}, p_{2}\right)$ is bilinear symmetric meromorphic on $\bar{\Sigma}_{q} \times \bar{\Sigma}_{q}$.

(2) The differential $B\left(p_{1}, p_{2}\right)$ is holomorphic everywhere except for a double pole along the diagonal $p_{1}=p_{2}$. If $z_{1}, z_{2}$ are local coordinates on $\bar{\Sigma}_{q} \times \bar{\Sigma}_{q}$, then

$$
B\left(z_{1}, z_{2}\right)=\left(\frac{1}{\left(z_{1}-z_{2}\right)^{2}}+f\left(z_{1}, z_{2}\right)\right) d z_{1} d z_{2},
$$

where $f\left(z_{1}, z_{2}\right)$ is holomorphic.

(3) $\int_{p_{1} \in A_{i}} B\left(p_{1}, p_{2}\right)=0$ for $i=1, \ldots, \mathfrak{g}$.

Let $p_{\alpha}(q)=\left(X_{\alpha}(q), Y_{\alpha}(q)\right)$ be the branch point of $X: \bar{\Sigma}_{q} \rightarrow \mathbb{C}^{*}$ such that

$$
\lim _{q \rightarrow 0}\left(X_{\alpha}(q), Y_{\alpha}(q)\right)=\left(X_{\alpha}, Y_{\alpha}\right) .
$$

Following [Eyn14, EO15], given $\alpha \in G^{*}$ and $d \in \mathbb{Z}_{\geqslant 0}$, define

$$
\theta_{d}^{\alpha}(p):=-(2 d-1) ! ! 2^{-d} \operatorname{Res}_{p^{\prime} \rightarrow p_{\alpha}} B\left(p, p^{\prime}\right) \zeta_{\alpha}^{-2 d-1} .
$$

(In this paper, we use the symbol $\theta_{d}^{\alpha}$ instead of the symbol $d \xi_{\alpha, d}$ in [Eyn11, EO15] because the 1 -form defined by the right-hand side of (6.11) is not necessarily exact.) Then $\theta_{d}^{\alpha}$ satisfies the following properties:

(1) The form $\theta_{d}^{\alpha}$ is a meromorphic 1 -form on $\bar{\Sigma}_{q}$ with a single pole of order $2 d+2$ at $p_{\alpha}$.

(2) In local coordinate $\zeta_{\alpha}$ near $p_{\alpha}$,

$$
\theta_{d}^{\alpha}=\left(-\frac{-(2 d+1) ! !}{2^{d} \zeta_{\alpha}^{2 d+2}}+f\left(\zeta_{\alpha}\right)\right) d \zeta_{\alpha}
$$

where $f\left(\zeta_{\alpha}\right)$ is analytic around $p_{\alpha}$. The residue of $\theta_{d}^{\alpha}$ at $p_{\alpha}$ is zero, so $\theta_{d}^{\alpha}$ is a differential of the second kind.

(3) $\int_{A_{i}} \theta_{d}^{\alpha}=0$ for $i=1, \ldots, \mathfrak{g}$. 


\section{All-GenUs OPEN-CLOSED MIRROR SYMMETRY}

The meromorphic 1-form $\theta_{d}^{\alpha}$ is characterized by the above properties; $\theta_{d}^{\alpha}$ can be viewed as a section in $H^{0}\left(\bar{\Sigma}_{q}, \omega_{\bar{\Sigma}_{q}}\left((2 d+2) p_{\alpha}\right)\right)$.

LEMma 6.2. Suppose that $f$ is a meromorphic function on $\bar{\Sigma}_{q}$ with simple poles at the ramification points $\left\{p_{\beta}: \beta \in G^{*}\right\}$ and is holomorphic on $\bar{\Sigma}_{q} \backslash\left\{p_{\beta}: \beta \in G^{*}\right\}$. Then

$$
d f=\sum_{\beta \in G^{*}} c_{\beta} \theta_{0}^{\beta}, \quad \text { where } \quad c_{\beta}=\lim _{p \rightarrow p_{\beta}} f \zeta_{\beta} .
$$

Proof. Let $\Delta \omega=d f-\sum_{\beta \in G^{*}} c_{\beta} \theta_{0}^{\beta}$. Then $\Delta \omega$ is a holomorphic 1-form on $\bar{\Sigma}_{\alpha}$ and $\int_{A_{i}} \Delta \omega=0$ for $i=1, \ldots, \mathfrak{g}$. So $\Delta \omega=0$.

Remark 6.3. In Lemma 6.2, different choice of $A$-cycles $A_{1}, \ldots, A_{\mathfrak{g}}$ will give different meromorphic 1-forms $\theta_{0}^{\beta}$, but the equality

$$
d f=\sum_{\beta \in G^{*}} c_{\beta} \theta_{0}^{\beta}
$$

still holds, where the coefficients $c_{\beta}=\lim _{p \rightarrow p_{\beta}} f \zeta_{\beta}$ do not depend on the choice of $A$-cycles.

We make the following observations:

(1) The form $d x=-d X / X$ is a meromorphic 1-form on $\bar{\Sigma}_{q}$ which is holomorphic on $\Sigma_{q}$. It has a simple zero at each of the $|G|$ ramification points $\left\{p_{\alpha}:, \alpha \in G^{*}\right\}$ and a simple pole at each of the $\mathfrak{n}$ punctures $\bar{p}_{1}, \ldots, \bar{p}_{\mathfrak{n}}$.

(2) The form $d y=-d Y / Y$ is a meromorphic 1-form on $\bar{\Sigma}_{q}$ and a holomorphic 1-form on $\Sigma_{q}$. It is non-zero at each of the $|G|$ ramification points and has at most a simple pole at each of the $\mathfrak{n}$ punctures.

(3) For $a=1, \ldots, \mathfrak{g}$, the form $\nabla_{\partial / \partial q_{a}} \Phi$ is a holomorphic 1 -form on $\bar{\Sigma}_{q}$ which is non-zero at each of the $|G|$ ramification point.

(4) For $a=\mathfrak{g}+1, \ldots, p$, the form $\nabla_{\partial / \partial q_{a}} \Phi$ is a meromorphic 1-form on $\bar{\Sigma}_{q}$ which is holomorphic on $\Sigma_{q}$. It is non-zero at each of the $|G|$ ramification points and has at most a simple pole at each of the $\mathfrak{n}$ punctures.

On the basis of the above observations, the following are meromorphic function on $\bar{\Sigma}_{q}$ satisfying the assumption of Lemma 6.2:

$$
\frac{d y}{d x}, \quad \frac{\nabla_{\partial / \partial q_{a}} \Phi}{d x}=\frac{\partial y}{\partial q_{a}},
$$

where $a=1, \ldots, p$ and $\alpha \in G^{*}$.

Proposition 6.4. We have

$$
d\left(\frac{d y}{d x}\right)=\frac{1}{2} \sum_{\beta \in G^{*}} h_{1}^{\beta}(q) \theta_{0}^{\beta}
$$

For $a=1, \ldots, p$,

$$
d\left(\frac{\nabla_{\partial / \partial q_{a}} \Phi}{d x}\right)=\frac{1}{2} \sum_{\beta \in G^{*}} \frac{X_{\beta}(q)^{m_{a}} Y_{\beta}(q)^{n_{a}-f m_{a}}}{(\partial H / \partial x)\left(X_{\beta}(q), Y_{\beta}(q), q\right)} h_{1}^{\beta}(q) \theta_{0}^{\beta} .
$$

Equation (6.12) was proved in [EO15, Appendix D]; we include it for completeness. 
Proof of Proposition 6.4. Near $p_{\beta}$,

$$
\begin{gathered}
\frac{d y}{d x} \zeta_{\beta}=\frac{\sum_{k=1}^{\infty} h_{k}^{\beta}(q) k\left(\zeta_{\beta}\right)^{k} d \zeta_{\beta}}{2 \zeta_{\beta} d \zeta_{\beta}}=\frac{1}{2} \sum_{k=1}^{\infty} h_{k}^{\beta}(q) k\left(\zeta_{\beta}\right)^{k-1} \\
\frac{\nabla_{\partial / \partial q_{a}} \Phi}{d x} \zeta_{\beta}=\frac{-X^{m_{a}} Y^{n_{a}-f m_{a}}}{(\partial H / \partial y)(X, Y, q)} \zeta_{\beta}=\frac{X^{m_{a}} Y^{n_{a}-f m_{a}}}{(\partial H / \partial x)(X, Y, q)} \cdot \frac{d y}{d x} \zeta_{\beta} .
\end{gathered}
$$

So

$$
\lim _{p \rightarrow p_{\beta}} \frac{d y}{d x} \zeta_{\beta}=\frac{h_{1}^{\beta}(q)}{2}, \quad \lim _{p \rightarrow p_{\beta}} \frac{\nabla_{\partial / \partial q_{a}} \Phi}{d x} \zeta_{\beta}=\frac{X_{\beta}(q)^{m_{a}} Y_{\beta}(q)^{n_{a}-f m_{a}}}{(\partial H / \partial x)\left(X_{\beta}(q), Y_{\beta}(q), q\right)} \frac{h_{1}^{\beta}(q)}{2} .
$$

The proposition follows from Lemma 6.2.

Proposition 6.5. We have

$$
\begin{gathered}
d\left(\frac{\nabla_{\partial / \partial \tau_{a}} \Phi}{d x}\right)=\left.\frac{1}{2} \sum_{\beta \in G^{*}}\left(\frac{\partial H}{\partial \tau_{a}} / \frac{\partial H}{\partial x}\right)\right|_{X=X_{\beta}(q), Y=Y_{\beta}(q)} \cdot h_{1}^{\beta}(q) \theta_{0}^{\beta}, \\
\nabla_{\partial / \partial \tau_{a}} \nabla_{\partial / \partial \tau_{b}} \Phi=\left.\frac{1}{2} \sum_{\beta \in G^{*}}\left(\frac{\partial H}{\partial \tau_{a}} / \frac{\partial H}{\partial x}\right) \cdot\left(\frac{\partial H}{\partial \tau_{b}} / \frac{\partial H}{\partial x}\right)\right|_{X=X_{\beta}(q), Y=Y_{\beta}(q)} \cdot h_{1}^{\beta}(q) \theta_{0}^{\beta} .
\end{gathered}
$$

Proof. Equation (6.14) follows from equation (6.13). It remains to prove (6.15).

By a special geometry property of the topological recursion ([EO15, Theorem 4.4], proved in $[\mathrm{EO} 07])$,

$$
\left(\nabla_{\partial / \partial \tau_{a}} \nabla_{\partial / \partial \tau_{b}} \Phi\right)(p)=\int_{p_{1} \in B_{a}} \int_{p_{2} \in B_{b}} \omega_{0,3}\left(p_{1}, p_{2}, p\right)
$$

where (see Example 7.13)

$$
\omega_{0,3}\left(p_{1}, p_{2}, p\right)=\sum_{\beta \in G^{*}} \frac{-1}{2 h_{1}^{\beta}} \theta_{0}^{\beta}\left(p_{1}\right) \theta_{0}^{\beta}\left(p_{2}\right) \theta_{0}^{\beta}(p) .
$$

So $\nabla_{\partial / \partial \tau_{a}} \nabla_{\partial / \partial \tau_{b}} \Phi$ is a linear combination of $\theta_{0}^{\beta}$ :

$$
\nabla_{\partial / \partial \tau_{a}} \nabla_{\partial / \partial \tau_{b}} \Phi=\sum_{\beta \in G^{*}} c_{\beta} \theta_{0}^{\beta},
$$

where the coefficient $c_{\beta}$ is given by $c_{\beta}=\operatorname{Res}_{\zeta_{\beta} \rightarrow 0}\left(\zeta_{\beta} \cdot \nabla_{\partial / \partial \tau_{a}} \nabla_{\partial / \partial \tau_{b}} \Phi\right)$. We have

$$
\nabla_{\partial / \partial \tau_{a}} \nabla_{\partial / \partial \tau_{b}} \Phi=\sum_{c, d} \frac{\partial q_{c}}{\partial \tau_{a}} \frac{\partial q_{d}}{\partial \tau_{b}} \nabla_{\partial / \partial q_{c}} \nabla_{\partial / \partial q_{d}} \Phi+\sum_{c} \frac{\partial^{2} q_{c}}{\partial \tau_{a} \partial \tau_{b}} \nabla_{\partial / \partial q_{c}} \Phi
$$

where $\nabla_{\partial / \partial q_{c}} \Phi$ is holomorphic on $\Sigma_{q}$, and

$$
\nabla_{\partial / \partial q_{c}} \nabla_{\partial / \partial q_{d}} \Phi=\left(-\frac{\partial H}{\partial q_{a}} \frac{\partial H}{\partial q_{b}} \frac{\partial^{2} H}{\partial y^{2}} /\left(\frac{\partial H}{\partial y}\right)^{3}+\left(\frac{\partial^{2} H}{\partial q_{b} \partial y} \frac{\partial H}{\partial q_{a}}+\frac{\partial^{2} H}{\partial q_{a} \partial y} \frac{\partial H}{\partial q_{b}}\right) /\left(\frac{\partial H}{\partial y}\right)^{2}\right) d x
$$

So

$$
\begin{aligned}
c_{\beta} & =\left.\frac{\partial H}{\partial \tau_{a}} \frac{\partial H}{\partial \tau_{b}}\right|_{X=X_{\beta}(q), Y=Y_{\beta}(q)} \cdot \operatorname{Res}_{\zeta_{\beta} \rightarrow 0}\left(\zeta_{\beta}\left(-\frac{\partial^{2} H}{\partial y^{2}}\right) /\left(\frac{\partial H}{\partial y}\right)^{3} d x\right) \\
& =\left.\frac{1}{2}\left(\frac{\partial H}{\partial \tau_{a}} / \frac{\partial H}{\partial x}\right) \cdot\left(\frac{\partial H}{\partial \tau_{b}} / \frac{\partial H}{\partial x}\right)\right|_{X=X_{\beta}(q), Y=Y_{\beta}(q)} \cdot h_{\beta}^{1}(q) \cdot
\end{aligned}
$$




\section{All-GenUS OPEN-CLOSED MIRROR SYMMETRY}

Given $\alpha, \beta \in G^{*}$ and $k, l \in \mathbb{Z}_{\geqslant 0}$, define $B_{k, l}^{\alpha, \beta}$ to be the coefficients of the expansion of $B\left(p_{1}, p_{2}\right)$ near $\left(p_{\alpha}, p_{\beta}\right) \in \bar{\Sigma}_{q} \times \bar{\Sigma}_{q}$ in coordinates $\zeta_{1}=\zeta_{\alpha}\left(p_{1}\right)$ and $\zeta_{2}=\zeta_{\beta}\left(p_{2}\right)$. Then

$$
B\left(p_{1}, p_{2}\right)=\left(\frac{\delta_{\alpha, \beta}}{\left(\zeta_{1}-\zeta_{2}\right)^{2}}+\sum_{k, l \in \mathbb{Z}_{\geqslant 0}} B_{k, l}^{\alpha, \beta} \zeta_{1}^{k} \zeta_{2}^{l}\right) d \zeta_{1} d \zeta_{2}
$$

We define

$$
\check{B}_{k, l}^{\alpha, \beta}=\frac{(2 k-1) ! !(2 l-1) ! !}{2^{k+l+1}} B_{2 k, 2 l}^{\alpha, \beta} .
$$

The following lemma is the differential of [Eyn11, Equation (D.4)] and holds globally on the compactified mirror curve.

LEMMA 6.6. We have

$$
\theta_{k+1}^{\alpha}=-d\left(\frac{\theta_{k}^{\alpha}}{d x}\right)-\sum_{\beta \in G^{*}} \check{B}_{k, 0}^{\alpha, \beta} \theta_{0}^{\beta} .
$$

Proof. We have the following Laurent series expansion of the meromorphic function $\theta_{k}^{\alpha} / d x$ near $p_{\beta}$ in the local coordinate $\zeta_{\beta}$ :

$$
\frac{\theta_{k}^{\alpha}}{d x}=\delta_{\alpha, \beta} \frac{-(2 d+1) ! !}{2^{d+1} \zeta_{\beta}^{2 d+3}}-\frac{\check{B}_{k, 0}^{\alpha, \beta}}{\zeta_{\beta}}+h\left(\zeta_{\beta}\right),
$$

where $h\left(\zeta_{\beta}\right)$ is a power series in $\zeta_{\beta}$. Set $\Delta \omega=\theta_{k+1}^{\alpha}+d\left(\theta_{k}^{\alpha} / d x\right)+\sum_{\beta \in G^{*}} \check{B}_{k, 0}^{\alpha, \beta} \theta_{0}^{\beta}$. Then $\Delta \omega$ is a holomorphic 1-form on $\bar{\Sigma}_{q}$, and $\int_{A_{i}} \Delta \omega=0$ for $i=1, \ldots, \mathfrak{g}$. So $\Delta \omega=0$.

Given $\alpha \in G^{*}$ and $k \in \mathbb{Z}_{>0}$, we define

$$
\hat{\xi}_{\alpha, k}:=(-1)^{k}\left(\frac{d}{d x}\right)^{k-1}\left(\frac{\theta_{0}^{\alpha}}{d x}\right)=\left(X \frac{d}{d X}\right)^{k-1}\left(X \frac{\theta_{0}^{\alpha}}{d X}\right),
$$

which is a meromorphic function on $\bar{\Sigma}_{q}$. We define $d \hat{\xi}_{\alpha, 0}:=\theta_{0}^{\alpha}$.

Lemma 6.7. We have

$$
\theta_{k}^{\alpha}=d \hat{\xi}_{\alpha, k}-\sum_{i=0}^{k-1} \sum_{\beta \in G^{*}} \check{B}_{k-1-i, 0}^{\alpha, \beta} d \hat{\xi}_{\beta, i} .
$$

Proof. We prove the result by induction on $k$. When $k=0$, we have $\theta_{0}^{\alpha}=d \hat{\xi}_{\alpha, 0}$ by definition. Suppose that the lemma holds for $k=d$. By Lemma 6.6,

$$
\begin{aligned}
\theta_{d+1}^{\alpha} & =-d\left(\frac{\theta_{d}^{\alpha}}{d x}\right)-\sum_{\beta \in G^{*}} \check{B}_{d, 0}^{\alpha, \beta} \theta_{0}^{\beta} \\
& =-d\left(\frac{d \hat{\xi}_{\alpha, d}}{d x}-\sum_{i=0}^{d-1} \sum_{\beta \in G^{*}} \check{B}_{d-1-i, 0}^{\alpha, \beta} \frac{d \hat{\xi}_{\beta, i}}{d x}\right)-\sum_{\beta \in G^{*}} \check{B}_{d, 0}^{\alpha, \beta} d \hat{\xi}_{\beta, 0} \\
& =d \hat{\xi}_{\alpha, d+1}-\sum_{i=0}^{d-1} \sum_{\beta \in G^{*}} \check{B}_{d-1-i, 0}^{\alpha, \beta} d \hat{\xi}_{\beta, i+1}-\sum_{\beta \in G^{*}} \check{B}_{d, 0}^{\alpha, \beta} d \hat{\xi}_{\beta, 0} \\
& =d \hat{\xi}_{\alpha, d+1}-\sum_{i=0}^{d} \sum_{\beta \in G^{*}} \check{B}_{d-i, 0}^{\alpha, \beta} d \hat{\xi}_{\beta, i} .
\end{aligned}
$$

The second equality follows from the induction hypothesis. So the lemma holds for $k=d+1$. 


\section{B. FAnG, C.-C. M. LiU And Z. ZonG}

\section{B-model topological string}

\subsection{Laplace transform}

The Laplace transform of a meromorphic 1-form $\lambda$ along a Lefschetz thimble $\gamma_{\alpha}$ is given by $\int_{z \in \gamma_{\alpha}} e^{-u x} \lambda$.

DeFinition 7.1. We have $f_{\beta}^{\alpha}(u, q):=\frac{e^{u a_{\alpha}}}{2 \sqrt{\pi u}} \int_{z \in \gamma_{\alpha}} e^{-u x} \theta_{0}^{\beta}$.

It is straightforward to check that $f_{\beta}^{\alpha}(u, q)=\delta_{\alpha \beta}+O\left(u^{-1}\right)$.

The Eynard-Orantin invariants $\left\{\omega_{g, n}\right\}$ of the mirror curve can be expressed as a graph sum involving $f_{\beta}^{\alpha}(u, q), \theta_{d}^{\beta}$, and descendant integrals over moduli spaces of stable curves [KO10, Eyn11, Eyn14, DOSS14]. The goal of this subsection and the next two subsections is to relate $f_{\beta}^{\alpha}(u, q)$ and $\theta_{d}^{\beta}$ to terms in the A-model graph sum. The following is our strategy:

1. In this section (Section 7.1), we relate $f_{\beta}^{\alpha}(u, q)$ to the following Laplace transforms:

$$
\int_{\gamma_{\alpha}} e^{-u x} \Phi, \quad \int_{\gamma_{\alpha}} e^{-u x} \nabla_{\partial / \partial \tau_{a}} \Phi, \quad \int_{\gamma_{\alpha}} e^{-u x} \nabla_{\partial / \partial \tau_{a}} \nabla_{\partial / \partial \tau_{b}} \Phi
$$

2. In Section 7.2, we evaluate the oscillatory integrals in the Landau-Ginzburg mirror of $\mathcal{X}=\left[\mathbb{C}^{3} / G\right]$ for any small $q$. These oscillatory integrals can be identified with the Laplace transforms in (7.1) by dimensional reduction.

3. In Section 7.3, we expand an antiderivative of $\theta_{0}^{\beta}$ near $X=0$ and relate it to $\widetilde{\xi}_{0}^{\beta}$ from Section 5.2.

By equation (6.12),

$$
\begin{aligned}
\int_{z \in \gamma_{\alpha}} e^{-u x} \Phi & =u^{-2} \int_{z \in \gamma_{\alpha}} e^{-u x} d\left(\frac{d y}{d x}\right)=\frac{1}{2} u^{-2} \sum_{\beta \in G^{*}} h_{1}^{\beta} \int_{z \in \gamma_{\alpha}} e^{-u x} d \xi_{\beta, 0} \\
& =\sqrt{\pi} u^{-3 / 2} e^{-u a_{\alpha}} \sum_{\beta \in G^{*}} h_{1}^{\beta} f_{\beta}^{\alpha}(u, q) .
\end{aligned}
$$

By equation (6.14),

$$
\begin{aligned}
\int_{z \in \gamma_{\alpha}} e^{-u x} \nabla_{\partial / \partial \tau_{a}} \Phi & =-u^{-1} \int_{z \in \gamma_{\alpha}} e^{-u x} d\left(\frac{\nabla_{\partial / \partial \tau_{a}} \Phi}{d x}\right) \\
& =-\left.\frac{1}{2} u^{-1} \sum_{\beta \in G^{*}}\left(\frac{\partial H}{\partial \tau_{a}} / \frac{\partial H}{\partial x}\right)\right|_{X=X_{\beta}, Y=Y_{\beta}} \cdot h_{1}^{\beta} \int_{z \in \gamma_{\alpha}} e^{-u x} \theta_{0}^{\beta} \\
& =-\left.\sqrt{\pi} u^{-1 / 2} e^{-u a_{\alpha}} \sum_{\beta \in G^{*}}\left(\frac{\partial H}{\partial \tau_{a}} / \frac{\partial H}{\partial x}\right)\right|_{X=X_{\beta}, Y=Y_{\beta}} \cdot h_{1}^{\beta}(q) f_{\beta}^{\alpha}(u, q) .
\end{aligned}
$$

By equation (6.15),

$$
\begin{gathered}
\int_{z \in \gamma_{\alpha}} e^{-u x} \nabla_{\partial / \partial \tau_{b}} \nabla_{\partial / \partial \tau_{a}} \Phi=\left.\frac{1}{2} \sum_{\beta \in G^{*}}\left(\frac{\partial H}{\partial \tau_{a}} / \frac{\partial H}{\partial x}\right) \cdot\left(\frac{\partial H}{\partial \tau_{b}} / \frac{\partial H}{\partial x}\right)\right|_{X=X_{\beta}, Y=Y_{\beta}} \cdot h_{1}^{\beta} \int_{z \in \gamma_{\alpha}} e^{-u x} \theta_{0}^{\beta} \\
=\left.\sqrt{\pi} u^{1 / 2} e^{-u a_{\alpha}} \sum_{\beta \in G^{*}}\left(\frac{\partial H}{\partial \tau_{a}} / \frac{\partial H}{\partial x}\right) \cdot\left(\frac{\partial H}{\partial \tau_{b}} / \frac{\partial H}{\partial x}\right)\right|_{X=X_{\beta}, Y=Y_{\beta}} \cdot h_{1}^{\beta}(q) f_{\beta}^{\alpha}(u, q) .
\end{gathered}
$$




\section{AlL-GENUS OPEN-CLOSED MIRROR SYMMETRY}

In the remainder of this subsection, we consider the limit $q \rightarrow 0$. We have

$$
\begin{gathered}
\lim _{q \rightarrow 0} h_{1}^{\beta}(q)=\frac{1}{|G|} \sqrt{\frac{-2}{w_{1} w_{2} w_{3}}}, \\
\left.\lim _{q \rightarrow 0}\left(\frac{\partial H}{\partial \tau_{a}} / \frac{\partial H}{\partial x}\right)\right|_{X=X_{\beta}, Y=Y_{\beta}}=\chi_{\beta}\left(h_{a}\right) \prod_{i=1}^{3} w_{i}^{c_{i}\left(h_{a}\right)},
\end{gathered}
$$

where $h_{a} \in G$ corresponds to $\left(m_{a}, n_{a}, 1\right) \in \operatorname{Box}(\sigma)$, so that age $\left(h_{a}\right)=1$.

We introduce some notation. For $h \in G$, let

$$
\nabla_{h} \Phi= \begin{cases}\Phi, & h=1, \\ \nabla_{\partial / \partial \tau_{a}} \Phi, & h=h_{a}, \\ \nabla_{\partial / \partial \tau_{b}} \nabla_{\partial / \partial \tau_{a}} \Phi, & h=h_{a} h_{b} \text { and } \operatorname{age}(h)=2 .\end{cases}
$$

Then

$$
\begin{aligned}
\lim _{q \rightarrow 0} \int_{z \in \gamma_{\alpha}} e^{-u x} \nabla_{h} \Phi= & \frac{\sqrt{-2 \pi}(-1)^{\operatorname{age}(h)}}{|G|} u^{\operatorname{age}(h)-3 / 2}\left(\prod_{i=1}^{3} w_{i}^{c_{i}(h)-1 / 2}\right) \\
& \cdot e^{u\left(\sqrt{-1} \vartheta_{\alpha}+\sum_{i=1}^{3} w_{i} \log w_{i}\right)} \sum_{\beta \in G^{*}} f_{\beta}^{\alpha}(u, 0) \chi_{\beta}(h) .
\end{aligned}
$$

We conclude the following.

Proposition 7.2.

$$
\begin{aligned}
f_{\beta}^{\alpha}(u, 0)= & \frac{1}{\sqrt{-2 \pi}} \exp \left(-\left(\sqrt{-1} \vartheta_{\alpha}+\sum_{i=1}^{3} w_{i} \log w_{i}\right) u\right) \\
& \cdot \sum_{h \in G} \chi_{\beta}\left(h^{-1}\right) \cdot(-1)^{\operatorname{age}(h)} u^{3 / 2-\operatorname{age}(h)} \prod_{i=1}^{3} w_{i}^{-c_{i}(h)+1 / 2} \cdot \lim _{q \rightarrow 0} \int_{\gamma_{\alpha}} e^{-u x} \nabla_{h} \Phi .
\end{aligned}
$$

We will evaluate $\lim _{q \rightarrow 0} \int_{\gamma_{\alpha}} e^{-u x} \nabla_{h} \Phi$ in the next subsection.

\subsection{Oscillatory integrals}

The equivariant Landau-Ginzburg mirror of a general toric orbifolds has been studied by Iritani [Iri09].

The mirror B-model to $\mathcal{X}=\left[\mathbb{C}^{3} / G\right]$ is a Landau-Ginzburg model $W_{q}:\left(\mathbb{C}^{*}\right)^{3} \rightarrow \mathbb{C}$, where

$$
W_{q}=X_{1}^{r} X_{2}^{-s-r f} X_{3}+X_{2}^{m} X_{3}+X_{3}+\sum_{a=1}^{p} q_{a} X_{1}^{m_{a}} X_{2}^{n_{a}-m_{a} f} X_{3} .
$$

Define $H=W / X_{3}$. Following Iritani [Iri09], the equivariantly perturbed B-model superpotential $\widetilde{W}_{q}$ is

$$
\widetilde{W}_{q}=W_{q}-u \log X_{1}
$$

We assume $w_{1}, w_{2}, u>0$ and $w_{3}<0$ as usual. Define

$$
t_{1}=X_{1}^{r} X_{2}^{-s-r f} X_{3}, \quad t_{2}=X_{2}^{m} X_{3}, \quad \hat{t}_{1}=X_{1}^{r} X_{2}^{-s-r f}, \quad \hat{t}_{2}=X_{2}^{m} .
$$




\section{B. FAnG, C.-C. M. LiU And Z. ZonG}

We use the notation in Section 6.5. For each critical point $p_{\alpha}(q)=\left(a_{\alpha}(q), b_{\alpha}(q)\right)$ on the mirror curve, we have

$$
\Im\left(a_{\alpha}(0)\right)=-\pi w_{3}-\vartheta_{\alpha}, \quad \Im\left(b_{\alpha}(0)\right)=\frac{\pi}{m}-\varphi_{\alpha}, \quad e^{\sqrt{-1} \vartheta_{\alpha}}=\chi_{\alpha}\left(\eta_{1}\right), \quad e^{\sqrt{-1} \varphi_{\alpha}}=\chi_{\alpha}\left(\eta_{2}\right),
$$

where $\Im(z)$ is the imaginary part of $z$. Notice that we have a preferred choice of the labeling of branch points by the elements in $G^{*}$ with $\vartheta_{1}=\varphi_{1}=0$. Let $C=-1+\sqrt{-1} \mathbb{R} \subset \mathbb{C}^{*}$, and define (Lagrangian) cycles $\Gamma_{\alpha, q}^{\mathrm{red}} \subset\left(\mathbb{C}^{*}\right)^{2}$ and $\Gamma_{\alpha, q} \subset\left(\mathbb{C}^{*}\right)^{3}$ by

$$
\Gamma_{\alpha, q}^{\mathrm{red}}:=\left(\mathbb{R}^{+} e^{-a_{\alpha}(q)}\right) \times\left(\mathbb{R}^{+} e^{-b_{\alpha}(q)}\right), \quad \Gamma_{\alpha, q}:=\Gamma_{\alpha, q}^{\mathrm{red}} \times C \subset\left(\mathbb{C}^{*}\right)^{3} .
$$

In the perturbed superpotential $\widetilde{W}_{q}$, the logarithm is defined in the following way: when $q=0$, we have $X_{3}<0, \Im\left(\log X_{1}\right)=\pi w_{3}+\vartheta_{\alpha}, \Im\left(\log X_{2}\right)=-\pi / m+\varphi_{\alpha}, \Im\left(\log X_{3}\right)=\pi$. Since the cycle $\Gamma_{\alpha, q}$ is contractible and deforms continuously with respect to $q$, the choice is fixed by these conditions on $\Gamma_{\alpha, q}$.

Define the oscillatory integral of $\widetilde{W}_{q}$ to be

$$
\widetilde{I}_{\alpha}^{\mathcal{X}}(u)=\int_{\Gamma_{\alpha, q}} e^{-\widetilde{W}_{q}} \frac{d X_{1}}{X_{1}} \frac{d X_{2}}{X_{2}} \frac{d X_{3}}{X_{3}} .
$$

Define $L_{\alpha}:=\left\{\left(q_{1}, \ldots, q_{p}\right) \in \mathbb{C}^{p}: q_{a} X_{\alpha}^{m_{a}} Y_{\alpha}^{n_{a}-m_{a} f} \in \mathbb{R}\right\}$, which is a totally real linear subspace of $\mathbb{C}^{p}$. We will view $\widetilde{I}_{\alpha}^{\mathcal{X}}(u)$ as a function of $q \in L_{\alpha} \cong \mathbb{R}^{p}$ and consider the power series expansion at $q=0$.

LEMmA 7.3. If $q \in L_{\alpha}$ is sufficiently small, then $\Gamma_{\alpha, q}^{\mathrm{red}}=\Gamma_{\alpha, 0}^{\mathrm{red}}$.

Proof. Consider the change of variables $X=X_{\alpha} A, Y=Y_{\alpha} B$, and $q_{a}=X_{\alpha}^{-m_{a}} Y_{\alpha}^{-n_{a}+m_{a} f} \epsilon_{a}$ for $a=1, \ldots, p$. Then $q=\left(q_{1}, \ldots, q_{p}\right) \in L_{\alpha}$ if and only if $\epsilon=\left(\epsilon_{1}, \ldots, \epsilon_{p}\right) \in \mathbb{R}^{p}$. We have

$$
\hat{t}_{1}=\frac{w_{1}}{w_{3}} A^{r} B^{-s-r f}, \quad \hat{t}_{2}=\frac{w_{2}}{w_{3}} B^{m}, \quad H(X, Y, q)=F(A, B, \epsilon), \quad Y \frac{\partial H}{\partial Y}=G(A, B, \epsilon),
$$

where

$$
\begin{aligned}
& F(A, B, \epsilon)=\frac{w_{1}}{w_{3}} A^{r} B^{-s-r f}+\frac{w_{2}}{w_{3}} B^{m}+1+\sum_{a=1}^{p} \epsilon_{a} A^{m_{a}} B^{n_{a}-f m_{a}}, \\
& G(A, B, \epsilon)=\frac{|G| w_{1} w_{2}}{w_{3}}\left(-A^{r} B^{-s-r f}+B^{m}\right)+\sum_{a=1}^{p}\left(n_{a}-f m_{a}\right) \epsilon_{a} A^{m_{a}} B^{n_{a}-f m_{a}}
\end{aligned}
$$

are $\mathbb{R}$-valued real analytic functions on $\mathbb{R}^{+} \times \mathbb{R}^{p}$. We have $F(1,1,0)=G(1,1,0)=0$, and the Jacobian $|(\partial(F, G) / \partial(A, B))(1,1,0)|=-|G|^{2} w_{1} w_{2}$ is non-zero. By the implicit function theorem, there exist real analytic functions $c, d: U \rightarrow \mathbb{R}^{+}$, where $U$ is an open neighborhood of 0 in $\mathbb{R}^{p}$, such that $c(0)=d(0)=1$ and $F(c(\epsilon), d(\epsilon), \epsilon)=G(c(\epsilon), d(\epsilon), \epsilon)=0$. If $\epsilon \in U$, then $e^{-a_{\alpha}(q)}=e^{-a_{\alpha}(0)} c(\epsilon)$ and $e^{-b_{\alpha}(q)}=e^{-b_{\alpha}(0)} d(\epsilon)$, so $\Gamma_{\alpha, q}^{\mathrm{red}}=\Gamma_{\alpha, 0}^{\mathrm{red}}$.

By Lemma 7.3 and its proof, if $q \in L_{\alpha}$ and $\left(X_{1}, X_{2}\right) \in \Gamma_{\alpha, q}^{\text {red }}$ then $\hat{t}_{1}, \hat{t}_{2} \in \mathbb{R}^{-}$and $H \in \mathbb{R}$. (Recall that $w_{1}, w_{2}>0$ and $w_{3}=-w_{1}-w_{2}<0$.) We first integrate out $\hat{t}_{1}$ and $\hat{t}_{2}$, and then integrate out $X_{3} \in C$. Recall that $h_{1}, \ldots, h_{p}$ are age 1 elements in $G$. Given $\vec{h}=\left(r_{1}, \ldots, r_{p}\right) \in$ $\mathbb{Z}^{\oplus p}$, where $r_{a} \geqslant 0$, define $c_{i}(\vec{h})=\sum_{a=1}^{p} r_{a} c_{i}\left(h_{a}\right) \in \mathbb{Q}$, and define $\chi_{\alpha}(\vec{h})=\chi_{\alpha}\left(\prod_{a=1}^{p} h_{a}^{r_{a}}\right) \in U(1)$. 


\section{All-GenUs OPEN-CLOSED MIRROR SYMMETRY}

Then $\widetilde{I}_{\alpha}^{\mathcal{X}}(u)$ is equal to

$$
\begin{aligned}
& \int_{\Gamma_{\alpha, q}}\left(\exp \left(-\sum_{a=1}^{p} q_{a} X_{1}^{m_{a}} X_{2}^{n_{a}-m_{a} f} X_{3}-t_{1}-t_{2}-X_{3}\right)\left(-\hat{t}_{1}\right)^{u w_{1}}\left(-\hat{t}_{2}\right)^{u w_{2}}\right. \\
& \left.\cdot e^{u\left(w_{1}+w_{2}+w_{3}\right)\left(\log X_{3}-\sqrt{-1} \pi\right)} e^{\sqrt{-1}\left(\vartheta_{\alpha}+\pi w_{3}\right) u}\right) \frac{d\left(-\hat{t}_{1}\right) d\left(-\hat{t}_{2}\right) d X_{3}}{|G|\left(-\hat{t}_{1}\right)\left(-\hat{t}_{2}\right) X_{3}} \\
& =\frac{-1}{|G|} e^{\sqrt{-1}\left(\vartheta_{\alpha}+\pi w_{3}\right) u} \sum_{\substack{\vec{h}=\left(r_{1}, \ldots, r_{p}\right) \\
r_{a} \in \mathbb{Z} \geqslant 0}} e^{\sqrt{-1} \pi c_{3}(\vec{h})} \chi_{\alpha}(\vec{h}) \prod_{a=1}^{p} \frac{\left(-q_{a}\right)^{r_{a}}}{r_{a} !} \\
& \cdot \int_{\Gamma_{\alpha, q}}\left(\left(-\hat{t}_{1}\right)^{c_{1}(\vec{h})+u w_{1}-1}\left(-\hat{t}_{2}\right)^{c_{2}(\vec{h})+u w_{2}-1}\right. \\
& \left.\cdot e^{\left(\log X_{3}-\sqrt{-1} \pi\right)\left(c_{1}(\vec{h})+c_{2}(\vec{h})+c_{3}(\vec{h})+u\left(w_{1}+w_{2}+w_{3}\right)-1\right)} e^{-\hat{t}_{1} X_{3}-\hat{t}_{2} X_{3}-X_{3}}\right) d\left(-\hat{t}_{1}\right) d\left(-\hat{t}_{2}\right) d X_{3} \\
& =\frac{-1}{|G|} e^{\sqrt{-1}\left(\vartheta_{\alpha}+\pi w_{3}\right) u} \sum_{\substack{\vec{h}=\left(r_{1}, \ldots, r_{p}\right) \\
r_{a} \in \mathbb{Z} \geqslant 0}} e^{\sqrt{-1} \pi c_{3}(\vec{h})} \chi_{\alpha}(\vec{h}) \prod_{a=1}^{p} \frac{\left(-q_{a}\right)^{r_{a}}}{r_{a} !} \\
& \cdot \int_{X_{3} \in C}\left(\int_{-\hat{t}_{1}>0} e^{-X_{3} \hat{t}_{1}}\left(-\hat{t}_{1}\right)^{c_{1}(\vec{h})+u w_{1}-1} d\left(-\hat{t}_{1}\right)\right)\left(\int_{-\hat{t}_{2}>0} e^{-\hat{t}_{2}}\left(-\hat{t}_{2}\right)^{c_{2}(\vec{h})+u w_{2}-1} d\left(-\hat{t}_{2}\right)\right) \\
& \cdot e^{-X_{3}} e^{\left(\log X_{3}-\sqrt{-1} \pi\right)\left(c_{1}(\vec{h})+c_{2}(\vec{h})+c_{3}(\vec{h})+u\left(w_{1}+w_{2}+w_{3}\right)-1\right)} d X_{3} \\
& =\frac{-1}{|G|} e^{\sqrt{-1}\left(\vartheta_{\alpha}+\pi w_{3}\right) u} \sum_{\substack{\vec{h}=\left(r_{1}, \ldots, r_{p}\right) \\
r_{a} \in \mathbb{Z} \geqslant 0}} e^{\sqrt{-1} \pi c_{3}(\vec{h})} \chi_{\alpha}(\vec{h}) \prod_{a=1}^{p} \frac{\left(-q_{a}\right)^{r_{a}}}{r_{a} !} \Gamma\left(u w_{1}+c_{1}(\vec{h})\right) \Gamma\left(u w_{2}+c_{2}(\vec{h})\right) \\
& \cdot \int_{X_{3} \in C} e^{-X_{3}} e^{\left(\log X_{3}-\sqrt{-1} \pi\right)\left(c_{3}(\vec{h})+u w_{3}-1\right)} d X_{3} \\
& =\frac{2 \pi \sqrt{-1}}{|G|} e^{\sqrt{-1}\left(\vartheta_{\alpha}+\pi w_{3}\right) u} \sum_{\substack{\vec{h}=\left(r_{1}, \ldots, r_{p}\right) \\
r_{a} \in \mathbb{Z} \geqslant 0}} e^{\pi \sqrt{-1} c_{3}(\vec{h})} \chi_{\alpha}(\vec{h}) \prod_{a=1}^{p} \frac{\left(-q_{a}\right)^{r_{a}}}{r_{a} !} \cdot \frac{\Gamma\left(u w_{1}+c_{1}(\vec{h})\right) \Gamma\left(u w_{2}+c_{2}(\vec{h})\right)}{\Gamma\left(-u w_{3}-c_{3}(\vec{h})+1\right)} .
\end{aligned}
$$

Here we use the following identity.

LEMMA 7.4. If $\Re z>0$, then

$$
\frac{\sqrt{-1}}{2 \pi}\left(\int_{C} e^{-z(\log (s)-\sqrt{-1} \pi)} e^{-s} d s\right)=\frac{1}{\Gamma(z)} .
$$

Proof. Hankel's representation of the reciprocal Gamma function says

$$
\frac{\sqrt{-1}}{2 \pi}\left(\int_{C_{\delta}} e^{-z(\log (t)-\sqrt{-1} \pi)} e^{-t} d t\right)=\frac{1}{\Gamma(z)},
$$

where $C_{\delta}$ is a Hankel contour (see Figure 2). The integrand is holomorphic on $\mathbb{C} \backslash[0, \infty)$, and

$$
\left|e^{-z(\log (t)-\sqrt{-1} \pi)} e^{-t}\right|=e^{-\Re(z) \log |s|+\Im(z)(\arg (t)-\pi)-\Re t} \leqslant e^{\pi \Im z}|t|^{-\Re z} e^{-(\Re z) t},
$$

so we may deform $C_{\delta}$ to the contour $C_{a, b}$ (see Figure 2) without changing the value of the contour 
integral. Therefore,

$$
\frac{\sqrt{-1}}{2 \pi}\left(\int_{C_{a, b}} e^{-z(\log (t)-\sqrt{-1} \pi)} e^{-t} d t\right)=\frac{1}{\Gamma(z)}
$$

for any $a, b \in(0, \infty)$. The estimate $(7.5)$ implies that contributions to the above contour integral from the two horizontal rays in $C_{a, b}$ tend to zero as $a, b \rightarrow+\infty$. The lemma follows from taking the limit of $(7.6)$ as $a, b \rightarrow+\infty$.
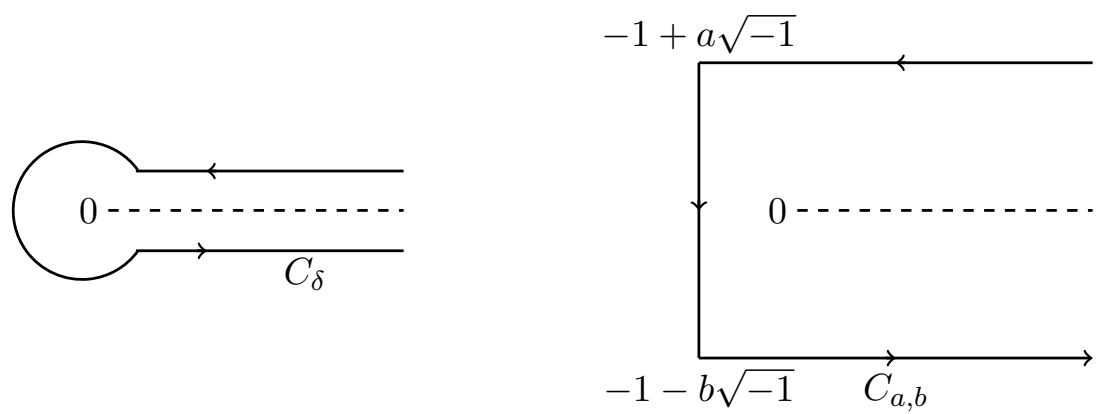

Figure 2. A Hankel contour $C_{\delta}$ and the contour $C_{a, b}(a, b>0)$

Remark 7.5. Let $f(t)$ be the inverse Laplace transform of $F(s)=\Gamma(z) / s^{z}$, where $z \in \mathbb{C}$ is a constant with $\Re z>0$. Lemma 7.4 implies that $f(t)=t^{z-1}$ for $t>0$.

By Hori-Iqbal-Vafa [HIV00], this oscillatory integral could be reduced to a Laplace transform on the mirror curve. The Landau-Ginzburg model on $\left(\mathbb{C}^{*}\right)^{3}$ is equivalent to a 5 -dimensional Landau-Ginzburg model, and the 5D model is again reduced to a Calabi-Yau threefold without potential. Further dimensional reduction reduces it to the mirror curve.

Introduce two variables $v^{+}, v^{-} \in \mathbb{C}$ and the cycles

$$
\widetilde{\Gamma}_{\alpha, q}=\Gamma_{\alpha, q} \times\left\{v^{+}=\overline{v^{-}}\right\}, \quad \widetilde{\Gamma}_{\alpha, q}^{\mathrm{red}}=\Gamma_{\alpha, q}^{\mathrm{red}} \times\left\{v^{+}=\overline{v^{-}}\right\} .
$$

The equation $H\left(X_{1}, X_{2}, q_{1}, \ldots, q_{p}\right)=0$ prescribes the mirror curve. Define the holomorphic volume form

$$
\Omega=\frac{d X_{1}}{X_{1}} \frac{d X_{2}}{X_{2}} \frac{d v^{-}}{v^{-}}=d x d y \frac{d v^{-}}{v^{-}} .
$$

We reduce the oscillatory integral to the mirror curve as follows:

$$
\begin{aligned}
\widetilde{I}_{\alpha}^{\mathcal{X}}(u) & =\frac{-1}{2 \sqrt{-1} \pi} \int_{\widetilde{\Gamma}_{\alpha, q}} e^{-X_{3}\left(H-v^{+} v^{-}\right)} e^{-u x} \frac{d X_{1}}{X_{1}} \frac{d X_{2}}{X_{2}} d X_{3} d v^{+} d v^{-} \\
& =\int_{\widetilde{\Gamma}_{\alpha, q}^{\mathrm{red}}} \delta\left(H-v^{+} v^{-}\right) e^{-u x} \frac{d X_{1}}{X_{1}} \frac{d X_{2}}{X_{2}} d v^{+} d v^{-} \\
& =-\int_{\widetilde{\Gamma}_{\alpha, q}^{\mathrm{red}} \cap\left\{H-v^{+} v^{-}=0\right\}} e^{-u x} \frac{d X_{1}}{X_{1}} \frac{d X_{2}}{X_{2}} \frac{d v^{-}}{v^{-}}
\end{aligned}
$$

The first identity comes from integrating out $v^{+}$and $v^{-}$(notice that on $C$, we have $\operatorname{Re}\left(X_{3}\right)<0$ ), using

$$
\int_{v^{+}=v^{-}} e^{X_{3} v^{+} v^{-}} d v^{+} d v^{-}=-\frac{2 \pi \sqrt{-1}}{X_{3}} .
$$




\section{All-Genus OPEN-ClOSED MIRROR SYMMETRY}

The second identity is the Fourier transform

$$
\int_{C} e^{-X_{3}\left(H-v^{+} v^{-}\right)} d X_{3}=\int_{-\infty}^{+\infty} e^{(1+s \sqrt{-1})\left(H-v^{+} v^{-}\right)}(-\sqrt{-1}) d s=-2 \pi \sqrt{-1} \delta\left(H-v^{+} v^{-}\right) .
$$

For the third identity, fixing $X_{1}$ and $X_{2}$ such that $H \geqslant 0$, and letting $v^{ \pm}=r e^{ \pm \sqrt{-1} \theta}$, we have

$$
\int_{v^{+}=\bar{v}^{-}} \delta\left(H-v^{+} v^{-}\right) d v^{+} d v^{-}=2 \pi \sqrt{-1} \int_{0}^{\infty} \delta\left(H-r^{2}\right) 2 r d r=2 \pi \sqrt{-1}=-\int_{\left|v^{-}\right|=H} \frac{d v^{-}}{v^{-}} .
$$

This integration is further reduced to the mirror curve $H\left(e^{-x}, e^{-y}\right)=0$ as follows:

$$
\widetilde{I}_{\alpha}^{\mathcal{X}}(u)=-\int_{\widetilde{\Gamma}_{\alpha, q}^{\mathrm{red}} \cap\left\{H-v^{+} v^{-}=0\right\}} e^{-u x} d x d y \frac{d v^{-}}{v^{-}}=2 \sqrt{-1} \pi \int_{\gamma_{\alpha}=\widetilde{\Gamma}_{\alpha, q}^{\mathrm{red}} \cap\left\{H=v^{-}=0\right\}} e^{-u x} y d x .
$$

Notice that we use the fact that $d\left(e^{-u x} y d x\left(d v^{-} / v^{-}\right)\right)=-e^{-u x} \Omega$ near $\widetilde{\Gamma}_{\alpha, q}^{\text {red }} \cap\left\{H-v^{+} v^{-}=0\right\}$. Therefore, we obtain the following formula.

TheOREM 7.6. The integral $\int_{\gamma_{\alpha}} e^{-u x} \Phi$ is equal to

$$
\frac{1}{|G|} e^{\sqrt{-1}\left(\vartheta_{\alpha}+\pi w_{3}\right) u} \sum_{\substack{\vec{h}=\left(r_{1}, \ldots, r_{p}\right) \\ r_{a} \in \mathbb{Z}_{\geqslant} \geqslant 0}} e^{\pi \sqrt{-1} c_{3}(\vec{h})} \chi_{\alpha}(\vec{h}) \prod_{a=1}^{p} \frac{\left(-q_{a}\right)^{r_{a}}}{r_{a} !} \cdot \frac{\Gamma\left(u w_{1}+c_{1}(\vec{h})\right) \Gamma\left(u w_{2}+c_{2}(\vec{h})\right)}{\Gamma\left(-u w_{3}-c_{3}(\vec{h})+1\right)} .
$$

Corollary 7.7. We have

$$
\lim _{q \rightarrow 0} \int_{\gamma_{\alpha}} e^{-u x} \nabla_{h} \Phi=\frac{e^{\sqrt{-1}\left(\vartheta_{\alpha}+\pi w_{3}\right) u}}{|G|}(-1)^{\operatorname{age}(h)} e^{\sqrt{-1} \pi c_{3}(h)} \chi_{\alpha}(h) \frac{\Gamma\left(u w_{1}+c_{1}(h)\right) \Gamma\left(u w_{2}+c_{2}(h)\right)}{\Gamma\left(-u w_{3}-c_{3}(h)+1\right)} .
$$

TheOREM 7.8. We have

$$
f_{\beta}^{\alpha}(u, 0)=\sum_{h \in G} \frac{\chi_{\alpha}(h) \chi_{\beta}\left(h^{-1}\right)}{|G|} \exp \left(\sum_{m \geqslant 1} \frac{(-1)^{m+1}}{m(m+1)} \sum_{i=1}^{3} B_{m+1}\left(c_{i}(h)\right)\left(w_{i} u\right)^{-m}\right) .
$$

Proof. By Proposition 7.2 and Corollary 7.7,

$$
f_{\beta}^{\alpha}(u, 0)=\sum_{h \in G} \frac{\chi_{\alpha}(h) \chi_{\beta}\left(h^{-1}\right)}{|G|} \cdot C_{h} \cdot \frac{\Gamma\left(u w_{1}+c_{1}(h)\right) \Gamma\left(u w_{2}+c_{2}(h)\right)}{\Gamma\left(-u w_{3}-c_{3}(h)+1\right)},
$$

where

$$
C_{h}=\frac{1}{\sqrt{-2 \pi}} \exp \left(-u \sum_{i=1}^{3} w_{i} \log w_{i}\right) \cdot u^{3 / 2-\operatorname{age}(h)} \prod_{i=1}^{3} w_{i}^{-c_{i}(h)+1 / 2} e^{\sqrt{-1} \pi\left(w_{3} u+c_{3}(h)\right)} .
$$


By the Stirling formula [KP11],

$$
\begin{aligned}
\log & \frac{\Gamma\left(w_{1} u+c_{1}(h)\right) \Gamma\left(w_{2} u+c_{2}(h)\right)}{\Gamma\left(-w_{3} u+1-c_{3}(h)\right)} \\
= & \left(w_{1} u+c_{1}(h)-\frac{1}{2}\right) \log \left(w_{1} u\right)+\left(w_{2} u+c_{2}(h)-\frac{1}{2}\right) \log \left(w_{2} u\right) \\
& -\left(-w_{3} u-c_{3}(h)+\frac{1}{2}\right) \log \left(-w_{3} u\right)+\frac{1}{2} \log (2 \pi) \\
& +\sum_{m \geqslant 1} \frac{(-1)^{m+1}}{m(m+1)} \sum_{i=1}^{3} B_{m+1}\left(c_{i}(h)\right)\left(w_{i} u\right)^{-m} \\
= & \log \sqrt{-2 \pi}-\sqrt{-1} \pi\left(w_{3} u+c_{3}(h)\right)+\left(\operatorname{age}(h)-\frac{3}{2}\right) \log u+\sum_{i=1}^{3}\left(c_{i}(h)-\frac{1}{2}\right) \log w_{i} \\
& +u \sum_{i=1}^{3} w_{i} \log w_{i}+\sum_{m \geqslant 1} \frac{(-1)^{m+1}}{m(m+1)} \sum_{i=1}^{3} B_{m+1}\left(c_{i}(h)\right)\left(w_{i} u\right)^{-m} \\
\frac{\Gamma\left(w_{1}\right.}{u} & \left.+c_{1}(h)\right) \Gamma\left(w_{2} u+c_{2}(h)\right) \\
& \Gamma\left(-w_{3} u+1-c_{3}(h)\right) \\
= & \sqrt{-2 \pi} e^{-\sqrt{-1} \pi\left(w_{3} u+c_{3}(h)\right)} u^{\operatorname{age}(h)-3 / 2} \prod_{i=1}^{3} w_{i}^{c_{i}(h)-1 / 2} \exp \left(u \sum_{i=1}^{3} w_{i} \log w_{i}\right) \\
& \cdot \exp \left(\sum_{m \geqslant 1} \frac{(-1)^{m+1}}{m(m+1)} \sum_{i=1}^{3} B_{m+1}\left(c_{i}(h)\right)\left(w_{i} u\right)^{-m}\right) \\
= & C_{h}^{-1} \cdot \exp \left(\sum_{m \geqslant 1} \frac{(-1)^{m+1}}{m(m+1)} \sum_{i=1}^{3} B_{m+1}\left(c_{i}(h)\right)\left(w_{i} u\right)^{-m}\right)
\end{aligned}
$$

Therefore,

$$
f_{\beta}^{\alpha}(u, 0)=\sum_{h \in G} \frac{\chi_{\alpha}(h) \chi_{\beta}\left(h^{-1}\right)}{|G|} \exp \left(\sum_{m \geqslant 1} \frac{(-1)^{m+1}}{m(m+1)} \sum_{i=1}^{3} B_{m+1}\left(c_{i}(h)\right)\left(w_{i} u\right)^{-m}\right) .
$$

Define

$$
\check{h}^{\alpha}(u, q):=\frac{u^{3 / 2}}{\sqrt{\pi}} e^{u a_{\alpha}} \int_{z \in \gamma_{\alpha}} e^{-u x} \Phi=\sum_{\beta \in G^{*}} f_{\beta}^{\alpha}(u, q) h_{1}^{\beta} .
$$

Corollary 7.9.

$$
\check{h}^{\alpha}(u, 0)=\frac{1}{|G|} \sqrt{\frac{-2}{w_{1} w_{2} w_{3}}} \exp \left(\sum_{m \geqslant 1} \frac{(-1)^{m+1}}{m(m+1)} \sum_{i=1}^{3} B_{m+1}\left(w_{i} u\right)^{-m}\right) .
$$

Proof. Recall that

for any $\beta \in G^{*}$, so

$$
\lim _{q \rightarrow 0} h_{1}^{\beta}=\frac{1}{|G|} \sqrt{\frac{-2}{w_{1} w_{2} w_{3}}}
$$

$$
\check{h}^{\alpha}(u, 0)=\frac{1}{|G|} \sqrt{\frac{-2}{w_{1} w_{2} w_{3}}} \sum_{\beta \in G^{*}} f_{\beta}^{\alpha}(u, 0) .
$$


By Theorem 7.8,

$$
\sum_{\beta \in G^{*}} f_{\beta}^{\alpha}(u, 0)=\sum_{h \in G} \frac{\chi_{\alpha}(h)}{|G|}\left(\sum_{\beta \in G^{*}} \chi_{\beta}\left(h^{-1}\right)\right) \exp \left(\sum_{m \geqslant 1} \frac{(-1)^{m+1}}{m(m+1)} \sum_{i=1}^{3} B_{m+1}\left(c_{i}(h)\right)\left(w_{i} u\right)^{-m}\right),
$$

where

$$
\sum_{\beta \in G^{*}} \chi_{\beta}\left(h^{-1}\right)=\sum_{\beta \in G^{*}} \chi_{\beta}\left(h^{-1}\right) \chi_{\beta}(1)=|G| \delta_{h^{-1}, 1}
$$

So

$$
\begin{aligned}
\check{h}^{\alpha}(u, 0) & =\frac{1}{|G|} \sqrt{\frac{-2}{w_{1} w_{2} w_{3}}} \chi_{\alpha}(1) \exp \left(\sum_{m \geqslant 1} \frac{(-1)^{m+1}}{m(m+1)} \sum_{i=1}^{3} B_{m+1}\left(c_{i}(1)\right)\left(w_{i} u\right)^{-m}\right) \\
& =\frac{1}{|G|} \sqrt{\frac{-2}{w_{1} w_{2} w_{3}}} \exp \left(\sum_{m \geqslant 1} \frac{(-1)^{m+1}}{m(m+1)} \sum_{i=1}^{3} B_{m+1}\left(w_{i} u\right)^{-m}\right) .
\end{aligned}
$$

\subsection{Inverse Laplace transform and expansion at $X=0$}

Recall that on the Lefschetz thimble $\gamma_{\alpha}$, the local coordinate is $\zeta_{\alpha}(q)$, and $x=a_{\alpha}(q)+\zeta_{\alpha}(q)^{2}$. We choose $\zeta_{\alpha}(q)$ such that when $\zeta_{\alpha}(0) \rightarrow-\infty$, the corresponding point on the mirror curve $\Sigma_{0}$ approaches a point with $X=0$ and $Y^{m}=-1$ (this is consistent with our choice $\left.h_{1}^{\alpha}(0)>0\right)$. Furthermore, we require that for any element $\alpha^{\prime} \in G^{*}$, its action on the compactified mirror curve $\bar{\Sigma}_{0}$ moves the end point $\zeta_{\alpha}=-\infty$ on $\gamma_{\alpha}$ to the end point $\zeta_{\alpha^{\prime} \alpha}=-\infty$ on $\gamma_{\alpha^{\prime} \alpha}$. (Here $\alpha^{\prime} \alpha \in G^{*}$ is the product of $\alpha, \alpha^{\prime} \in G^{*}$; that is, $\chi_{\alpha^{\prime} \alpha}(h)=\chi_{\alpha^{\prime}}(h) \chi_{\alpha}(h)$ for all $h \in G$.)

We label the $m$ points with $X=0$ and $Y^{m}=-1$ on $\bar{\Sigma}_{0}$ by elements in $\boldsymbol{\mu}_{m}^{*} \cong \mathbb{Z}_{m}$ as follows: $\bar{p}_{\ell}:=\left(0, e^{\pi \sqrt{-1}(2 \ell+1) / m}\right)$ for $\ell \in \mathbb{Z}_{m}$. With our convention, if $\chi_{\alpha}=\chi_{1}^{j} \chi_{2}^{\ell}$, then the end point $\zeta_{\alpha}=-\infty$ is $\bar{p}_{\ell-1}$.

Every 1-form on $\gamma_{\alpha}$ is exact, so there exists a function $\xi_{\beta, 0}^{\alpha}$ on $\gamma_{\alpha}$ such that $d \xi_{\beta, 0}^{\alpha}=\left.\theta_{0}^{\beta}\right|_{\gamma_{\alpha}}$. The Laplace transform of $\theta_{0}^{\beta}$ along $\gamma_{\alpha}$ is

$$
\begin{aligned}
f_{\beta}^{\alpha}(u) & =\frac{e^{u a_{\alpha}}}{2 \sqrt{\pi u}} \int_{z \in \gamma_{\alpha}} e^{-u x(z)} \theta_{0}^{\beta}=\frac{\sqrt{u}}{2 \sqrt{\pi}} \int_{z \in \gamma_{\alpha}} e^{-u\left(x(z)-a_{\alpha}\right)} \xi_{\beta, 0}^{\alpha} d\left(x(z)-a_{\alpha}\right) \\
& =\frac{\sqrt{u}}{2 \sqrt{\pi}} \int_{x-a_{\alpha} \in \mathbb{R}^{+}} e^{-u\left(x-a_{\alpha}\right)}\left(\xi_{\beta, 0}^{\alpha+}(x)-\xi_{\beta, 0}^{\alpha-}(x)\right) d\left(x-a_{\alpha}\right)
\end{aligned}
$$

where $\xi_{\beta, 0}^{\alpha \pm}(x)=\xi_{\beta, 0}^{\alpha}\left(x, y\left( \pm \sqrt{x-a_{\alpha}}\right)\right) \in L^{2}(0, \infty)$. From the proof of Theorem 7.8

$$
\begin{aligned}
f_{\beta}^{\alpha}(u, 0)= & \frac{e^{-u \sum_{i=1}^{3} w_{i} \log w_{i}}}{\sqrt{-2 \pi}} \sum_{h \in G}\left(e^{\sqrt{-1} \pi\left(w_{3} u+c_{3}(h)\right)} \frac{\chi_{\alpha}(h) \chi_{\beta}\left(h^{-1}\right)}{|G|}\right. \\
& \left.\cdot u^{3 / 2-\operatorname{age}(h)} \cdot \prod_{i=1}^{3} w_{i}^{1 / 2-c_{i}(h)} \frac{\Gamma\left(w_{1} u+c_{1}(h)\right) \Gamma\left(w_{2} u+c_{2}(h)\right)}{\Gamma\left(-w_{3} u+1-c_{3}(h)\right)}\right) .
\end{aligned}
$$




\section{B. FANG, C.-C. M. LIU AND Z. ZonG}

So the "classical Laplace transform" is

$$
\begin{gathered}
\int_{x-a_{\alpha} \in \mathbb{R}^{+}} e^{-u\left(x-a_{\alpha}\right)}\left(\left.\xi_{\beta, 0}^{\alpha}\right|_{q=0}\right) d\left(x-a_{\alpha}\right) \\
=-\sqrt{-2} e^{-u \sum_{i=1}^{3} w_{i} \log w_{i}} \sum_{h \in G}\left(e^{\sqrt{-1} \pi\left(w_{3} u+c_{3}(h)\right)} \frac{\chi_{\alpha}(h) \chi_{\beta}\left(h^{-1}\right)}{|G|}\right. \\
\left.\cdot u^{1-\operatorname{age}(h)} \prod_{i=1}^{3} w_{i}^{1 / 2-c_{i}(h)} \frac{\Gamma\left(w_{1} u+c_{1}(h)\right) \Gamma\left(w_{2} u+c_{2}(h)\right)}{\Gamma\left(-w_{3} u+1-c_{3}(h)\right)}\right) .
\end{gathered}
$$

By the inverse Laplace transform formula,

$$
\begin{aligned}
-\lim _{q \rightarrow 0} \xi_{\beta, 0}^{\alpha-}= & -\sqrt{-2} \sum_{\substack{\left(d_{0}, h\right) \in \mathbb{Z} \times G \\
d_{0} / r-c_{1}(h) \in \mathbb{Z} \geqslant 0}} \operatorname{Res}_{u=-d_{0}}\left(\Gamma\left(w_{1} u+c_{1}(h)\right)\right)\left(e^{u\left(x-a_{\alpha}-\sum_{i=1}^{3} w_{i} \log w_{i}\right)} e^{\sqrt{-1} \pi\left(w_{3} u+c_{3}(h)\right)}\right. \\
& \left.\cdot \frac{\chi_{\alpha}(h) \chi_{\beta}\left(h^{-1}\right)}{|G|} u^{1-\operatorname{age}(h)} \prod_{i=1}^{3} w_{i}^{1 / 2-c_{i}(h)} \frac{\Gamma\left(w_{2} u+c_{2}(h)\right)}{\Gamma\left(-w_{3} u+1-c_{3}(h)\right)}\right)\left.\right|_{u=-d_{0}}, \\
\lim _{q \rightarrow 0} \xi_{\beta, 0}^{\alpha+}= & -\sqrt{-2} \sum_{\substack{\left(d_{0}, h\right) \in \mathbb{Z} \times G \\
w_{2} d_{0}-c_{2}(h) \in \mathbb{Z} \geqslant 0}} \operatorname{Res}_{u=-d_{0}}\left(\Gamma\left(w_{2} u+c_{2}(h)\right)\right)\left(e^{u\left(x-a_{\alpha}-\sum_{i=1}^{3} w_{i} \log w_{i}\right)} e^{\sqrt{-1} \pi\left(w_{3} u+c_{3}(h)\right)}\right. \\
& \left.\cdot \frac{\chi_{\alpha}(h) \chi_{\beta}\left(h^{-1}\right)}{|G|} u^{1-\operatorname{age}(h)} \prod_{i=1}^{3} w_{i}^{\frac{1}{2}-c_{i}(h)} \frac{\Gamma\left(w_{1} u+c_{1}(h)\right)}{\Gamma\left(-w_{3} u+1-c_{3}(h)\right)}\right)\left.\right|_{u=-d_{0}} .
\end{aligned}
$$

Remark 7.10. We obtain $\xi_{\beta, 0}^{\alpha+}$ and $\xi_{\beta, 0}^{\alpha-}$ by taking residues around the poles of $\Gamma\left(w_{1} u+c_{1}(h)\right)$ and $\Gamma\left(w_{2} u+c_{2}(h)\right)$. When both $w_{1} u+c_{1}(h)$ and $w_{2} u+c_{2}(h)$ are non-positive integers, the sum of the respective terms in $\xi_{\beta, 0}^{\alpha+}-\xi_{\beta, 0}^{\alpha-}$ is the residue.

We are interested in the expansion of $\xi_{\beta, 0}^{\alpha}$ at $\zeta=-\infty$, that is, the expansion of $\xi_{\beta, 0}^{\alpha-}$ at $X=0$,

$$
\begin{aligned}
\lim _{q \rightarrow 0} \xi_{\beta, 0}^{\alpha-}= & \sqrt{-2} \sum_{\substack{\left(d_{0}, k\right) \in \mathbb{Z}_{\geqslant 0} \times \mathbb{Z}_{m} \\
h=h\left(d_{0}, k\right)}} e^{-d_{0} x} \chi_{\alpha}\left(\eta_{1}^{-d_{0}}\right) e^{-\sqrt{-1} \pi\left(d_{0} w_{3}-c_{3}(h)\right)} \\
& \cdot \frac{\chi_{\alpha}(h) \chi_{\beta}\left(h^{-1}\right)}{|G|}\left(-d_{0}\right)^{1-\operatorname{age}(h)} \prod_{i=1}^{3} w_{i}^{1 / 2-c_{i}(h)} \frac{(-1)^{\left\lfloor d_{0} / r\right\rfloor} \Gamma\left(-d_{0} w_{2}+c_{2}(h)\right)}{\Gamma\left(d_{0} w_{1}-c_{1}(h)+1\right) \Gamma\left(d_{0} w_{3}+1-c_{3}(h)\right)} \\
= & \sqrt{-2} \sum_{\substack{\left(d_{0}, k\right) \in \mathbb{Z}_{\geqslant 0} \times \mathbb{Z}_{m} \\
h=h\left(d_{0}, k\right)}} X^{d_{0}} e^{-\sqrt{-1} \pi\left(d_{0} w_{3}-c_{3}(h)\right)} \frac{\chi_{\beta}\left(h^{-1}\right)}{m}\left(\frac{1}{d_{0}}\right)^{\operatorname{age}(h)-1} \\
& \cdot \prod_{i=1}^{3} w_{i}^{1 / 2-c_{i}(h)} \frac{\Gamma\left(d_{0}\left(w_{1}+w_{2}\right)+c_{3}(h)\right)}{\Gamma\left(d_{0} w_{1}-c_{1}(h)+1\right) \Gamma\left(d_{0} w_{2}-c_{2}(h)+1\right)} \chi_{\alpha}\left(\eta_{2}^{-k}\right) .
\end{aligned}
$$

This is the expansion of $\lim _{q \rightarrow 0} \xi_{\beta, 0}^{\alpha}$ at $X=0$ and $Y=e^{-\sqrt{-1} \pi / m} \chi_{\alpha}\left(\eta_{2}\right)$. If $\alpha=\chi_{1}^{j} \chi_{2}^{\ell}$, then

$$
\chi_{\alpha}\left(\eta_{2}^{-k}\right)=e^{-2 \sqrt{-1} \pi k \ell / m}
$$

depends only on $\ell \in\{0,1, \ldots, m-1\}$. Let $\xi_{\beta, 0}^{\ell-}$ be the expansion of $\xi_{\beta, 0}^{\alpha}$ at $\bar{p}_{\ell}=\left(0, e^{\pi \sqrt{-1}(2 \ell+1) / m}\right)$ 
for $\alpha=\chi_{1}^{j} \chi_{2}^{\ell+1}$. Define

$$
\psi_{\ell}:=\frac{1}{m} \sum_{k=0}^{m-1} \omega_{m}^{-k \ell} \mathbf{1}_{k / m}^{\prime}, \quad \ell=0,1, \ldots, m-1,
$$

where $\omega_{m}=e^{2 \pi \sqrt{-1} / m}$. Then $\left\{\psi_{0}, \ldots, \psi_{m-1}\right\}$ is a canonical basis of $H_{\mathrm{CR}}^{*}\left(\mathcal{B} \boldsymbol{\mu}_{m} ; \mathbb{C}\right)$. Define

$$
\xi_{0}^{\beta}:=\sum_{\ell=0}^{m-1} \xi_{\beta, 0}^{\ell-} \psi_{\ell}
$$

which takes values in $H_{\mathrm{CR}}^{*}\left(\mathcal{B} \boldsymbol{\mu}_{m} ; \mathbb{C}\right)$. Compare with the definition of $\widetilde{\xi}_{0}^{\beta}$ in Section 5.2; we obtain the following identity.

Proposition 7.11. We have

$$
\lim _{q \rightarrow 0} \xi_{0}^{\beta}=-\left.\frac{1}{|G|} \sqrt{\frac{-2}{w_{1} w_{2} w_{3}}} \widetilde{\xi}_{0}^{\beta}\right|_{v=1} .
$$

\subsection{Eynard-Orantin topological recursion}

Let $\omega_{g, n}$ be defined recursively by the Eynard-Orantin topological recursion [EO07]:

$$
\omega_{0,1}=0, \quad \omega_{0,2}=B\left(z_{1}, z_{2}\right) .
$$

When $2 g-2+n>0$,

$$
\begin{aligned}
\omega_{g, n}\left(p_{1}, \ldots, p_{n}\right)= & \sum_{\alpha \in G^{*}} \operatorname{Res}_{p \rightarrow p_{\alpha}} \frac{\int_{\xi=p}^{\bar{p}} B\left(p_{n}, \xi\right)}{2(\Phi(p)-\Phi(\bar{p}))}\left(\omega_{g-1, n+1}\left(p, \bar{p}, p_{1}, \ldots, p_{n-1}\right)\right. \\
& \left.+\sum_{g_{1}+g_{2}=g} \sum_{\substack{I \cup J=\{1, \ldots, n-1\} \\
I \cap J=\emptyset}} \omega_{g_{1},|I|+1}\left(p, p_{I}\right) \omega_{g_{2},|J|+1}\left(\bar{p}, p_{J}\right)\right),
\end{aligned}
$$

where $p, \bar{p} \rightarrow p_{\alpha}, X(p)=X(\bar{p})$, and $p \neq \bar{p}$.

The B-model invariants $\omega_{g, n}$ can be expressed as sums over labeled graphs involving intersection numbers on moduli spaces of stable curves [KO10, Eyn11, Eyn14, DOSS14]. We will use the formula stated in [DOSS14, Theorem 3.7], which is equivalent to the formula in [Eyn11, Theorem 5.1]. To state this graph sum, we introduce some definitions. Following Eynard [Eyn11], define the Laplace transform of the Bergman kernel $B\left(z_{1}, z_{2}\right)$

$$
\check{B}^{\alpha, \beta}(u, v, q):=\frac{u v}{u+v} \delta_{\alpha, \beta}+\frac{\sqrt{u v}}{2 \pi} e^{u a_{\alpha}+v a_{\beta}} \int_{z_{1} \in \gamma_{\alpha}} \int_{z_{2} \in \gamma_{\beta}} B\left(z_{1}, z_{2}\right) e^{-u x\left(z_{1}\right)-v x\left(z_{2}\right)},
$$

where $\alpha, \beta \in G^{*}$. By [Eyn11, equation (B.9)],

$$
\check{B}^{\alpha, \beta}(u, v, q)=\frac{u v}{u+v}\left(\delta_{\alpha, \beta}-\sum_{\gamma \in G^{*}} f_{\gamma}^{\alpha}(u, q) f_{\gamma}^{\beta}(v, q)\right) .
$$

Let $\check{B}_{k, l}^{\alpha, \beta}(q)$ be defined by (6.16). Then $\check{B}^{\alpha, \beta}(u, v, q)=\sum_{k, l} \check{B}_{k, l}^{\alpha, \beta}(q) u^{-k} v^{-l}$. Let $\check{h}_{k}^{\alpha}(q)$ be defined by

$$
\check{h}^{\alpha}(u, q)=\sum_{k} \check{h}_{k}^{\alpha}(q) u^{-k}
$$

where $\check{h}^{\alpha}$ is defined by (7.7). 
B. FAnG, C.-C. M. LiU And Z. Zong

Given a labeled graph $\vec{\Gamma}=(\Gamma, g, \alpha, k) \in \boldsymbol{\Gamma}_{g, 0, n}(\mathcal{B} G)$ with $L^{O}(\Gamma)=\left\{l_{1}, \ldots, l_{n}\right\}$, we define its weight to be

$$
\begin{aligned}
w(\vec{\Gamma})= & (-1)^{g(\vec{\Gamma})-1} \prod_{v \in V(\Gamma)}\left(\frac{h_{1}^{\alpha}}{\sqrt{-2}}\right)^{2-2 g-\operatorname{val}(v)}\left\langle\prod_{h \in H(v)} \tau_{k(h)}\right\rangle_{g(v)} \prod_{e \in E(\Gamma)} \check{B}_{k(e), l(e)}^{\alpha\left(v_{1}(e)\right), \alpha\left(v_{2}(e)\right)}(e) \\
& \cdot \prod_{j=1}^{n} \frac{1}{\sqrt{-2}} \theta_{k\left(l_{j}\right)}^{\alpha\left(l_{j}\right)}\left(z_{j}\right) \prod_{l \in \mathcal{L}^{1}(\Gamma)}\left(-\frac{1}{\sqrt{-2}}\right) \check{h}_{k(l)}^{\alpha(l)}(l) .
\end{aligned}
$$

In our notation, [DOSS14, Theorem 3.7] is equivalent to the following.

TheOrem 7.12. For $2 g-2+n>0$, we have

$$
\omega_{g, n}=\sum_{\Gamma \in \boldsymbol{\Gamma}_{g, 0, n}(\mathcal{B} G)} \frac{w(\vec{\Gamma})}{|\operatorname{Aut}(\vec{\Gamma})|} .
$$

Example 7.13 (Pair of pants).

$$
\omega_{0,3}\left(p_{1}, p_{2}, p_{3}\right)=\sum_{\alpha \in G^{*}} \frac{1}{2 h_{1}^{\alpha}} \theta_{0}^{\alpha}\left(p_{1}\right) \theta_{0}^{\alpha}\left(p_{2}\right) \theta_{0}^{\alpha}\left(p_{3}\right) .
$$

We now consider the unstable case $(g, n)=(0,2)$. Recall that $d x=-d X / X$ is a meromorphic 1 -form on $\bar{\Sigma}_{q}$, and $d / d x=-X d / d X$ is a meromorphic vector field on $\bar{\Sigma}_{q}$. Define

$$
C\left(z_{1}, z_{2}\right):=\left(-\frac{\partial}{\partial x}\left(z_{1}\right)-\frac{\partial}{\partial x}\left(z_{2}\right)\right)\left(\frac{\omega_{0,2}}{d x_{1} d x_{2}}\right)\left(z_{1}, z_{2}\right) d\left(x\left(z_{1}\right)\right)\left(d x\left(z_{2}\right)\right) .
$$

Then $C\left(z_{1}, z_{2}\right)$ is meromorphic on $\left(\bar{\Sigma}_{q}\right)^{2}$ and holomorphic on $\left(\bar{\Sigma}_{q} \backslash\left\{p_{\alpha}: \alpha \in G^{*}\right\}\right)^{2}$. It only has double poles on $\left\{p_{\alpha}: \alpha \in G^{*}\right\} \times \bar{\Sigma}_{q}$ and $\bar{\Sigma}_{q} \times\left\{p_{\beta}: \beta \in G^{*}\right\}$.

Lemma 7.14. We have

$$
C\left(z_{1}, z_{2}\right)=\frac{1}{2} \sum_{\gamma \in G^{*}} \theta_{0}^{\gamma}\left(z_{1}\right) \theta_{0}^{\gamma}\left(z_{2}\right) .
$$

Proof. First, we show that the Laplace transforms of both sides are asymptotically equal. For any $\alpha, \beta \in G^{*}$,

$$
\begin{aligned}
& \int_{z_{1} \in \gamma_{\alpha}} \int_{z_{2} \in \gamma_{\beta}} e^{-u\left(x\left(z_{1}\right)-a_{\alpha}\right)-v\left(x\left(z_{2}\right)-a_{\beta}\right)} C\left(z_{1}, z_{2}\right) \\
& =(-u-v) \int_{z_{1} \in \gamma_{\alpha}} \int_{z_{2} \in \gamma_{\beta}} e^{-u\left(x\left(z_{1}\right)-a_{\alpha}\right)-v\left(x\left(z_{2}\right)-a_{\beta}\right)} \omega_{0,2} \\
& \sim 2 \pi \sqrt{u v} \sum_{\gamma \in G^{*}} f_{\gamma}^{\alpha}(u, q) f_{\gamma}^{\beta}(v, q) \\
& \sim \frac{1}{2} \int_{z_{1} \in \gamma_{\alpha}} \int_{z_{2} \in \gamma_{\beta}} e^{-u\left(x\left(z_{1}\right)-a_{\alpha}\right)-v\left(x\left(z_{2}\right)-a_{\beta}\right)} \theta_{0}^{\gamma}\left(z_{1}\right) \theta_{0}^{\gamma}\left(z_{2}\right) .
\end{aligned}
$$

Define their difference

$$
\omega=C\left(z_{1}, z_{2}\right)-\frac{1}{2} \sum_{\gamma \in G^{*}} \theta_{0}^{\gamma}\left(z_{1}\right) \theta_{0}^{\gamma}\left(z_{2}\right) ;
$$

its Laplace transform at $\gamma_{\alpha} \times \gamma_{\beta}$ for any $\alpha, \beta \in G^{*}$ vanishes. For $i=1, \ldots, \mathfrak{g}$,

$$
\int_{p_{2} \in A_{i}} \omega_{0,2}\left(p_{1}, p_{2}\right)=0, \quad \int_{A_{i}} \theta_{0}^{\alpha}=0 .
$$




\section{All-GenUs OPEN-CLOSED MIRROR SYMMETRY}

Then

$$
\int_{p_{2} \in A_{i}} \omega\left(p_{1}, p_{2}\right)=0, \quad \int_{p_{2} \in A_{i}} \operatorname{Res}_{p_{1} \rightarrow p_{\alpha}} \zeta_{1, \alpha}\left(p_{1}\right) \omega\left(p_{1}, p_{2}\right)=0
$$

for all $i=1, \ldots, \mathfrak{g}$. Notice that the 1 -form

$$
\operatorname{Res}_{p_{1} \rightarrow p_{\alpha}} \zeta_{1, \alpha}\left(p_{1}\right) \omega\left(p_{1}, p_{2}\right)=\left[u^{0}\right] \frac{-1}{2 \sqrt{\pi u}} \int_{p_{1} \in \gamma_{\alpha}} e^{-u\left(x\left(p_{1}\right)-a_{\alpha}\right)} \omega\left(p_{1}, p_{2}\right)
$$

is a well-defined holomorphic form on $\bar{\Sigma}_{q}$. It has no poles; otherwise, any possible double pole at $p_{\beta}$ implies a non-zero Laplace transform of $\omega$ at $\gamma_{\alpha} \times \gamma_{\beta}$. It follows from the vanishing A-cycles integrals that

$$
\underset{p_{1} \rightarrow p_{\alpha}}{\operatorname{Res}} \zeta_{1, \alpha}\left(p_{1}\right) \omega\left(p_{1}, p_{2}\right)=0
$$

for any $\alpha \in G^{*}$, and then $\omega$ does not have any poles since it is symmetric and could only have double poles at the ramification points. Therefore, by the vanishing A-periods of $\omega$, we know that $\omega=0$.

\subsection{B-model potentials}

Choose $\delta>0$ and $\epsilon>0$ sufficiently small that for $|q|<\epsilon$, the meromorphic function $X: \bar{\Sigma}_{q} \rightarrow$ $\mathbb{C} \cup\{\infty\}$ restricts to an isomorphism

$$
X_{q}^{\ell}: D_{q}^{\ell} \rightarrow D_{\delta}=\{X \in \mathbb{C}:|X|<\delta\},
$$

where $D_{q}^{\ell}$ is an open neighborhood of $\bar{p}_{\ell} \in X^{-1}(0)$ and $\ell=0, \ldots, m-1$. Define

$$
\rho_{q}^{\ell_{1}, \ldots, \ell_{n}}:=\left(X_{q}^{\ell_{1}}\right)^{-1} \times \cdots \times\left(X_{q}^{\ell_{n}}\right)^{-1}:\left(D_{\delta}\right)^{n} \rightarrow D_{q}^{\ell_{1}} \times \cdots \times D_{q}^{\ell_{n}} \subset\left(\bar{\Sigma}_{q}\right)^{n} .
$$

(1) (Disk invariants) At $q=0$, we have $Y\left(\bar{p}_{\ell}\right)^{m}=-1$ for $\ell=0, \ldots, m-1$. When $\epsilon$ and $\delta$ are sufficiently small, $Y\left(\rho_{q}^{\ell}(X)\right) \in \mathbb{C} \backslash[0, \infty)$. Choose a branch of logarithm log: $\mathbb{C} \backslash[0, \infty) \rightarrow$ $(0,2 \pi)$, and define $y_{q}^{\ell}(X)=-\log Y\left(\rho_{q}^{\ell}(X)\right)$. The function $y_{q}^{\ell}(X)$ depends on the choice of logarithm, but $y_{q}^{\ell}(X)-y_{q}^{\ell}(0)$ does not. The form $d x=-d X / X$ is a meromorphic 1 -form on $\mathbb{C}$ with a simple pole at $X=0$, and $\left(y_{q}^{\ell}(X)-y_{q}^{\ell}(0)\right) d x$ is a holomorphic 1-form on $D_{\delta}$. Define the $B$-model disk potential by

$$
\check{F}_{0,1}(q ; X):=\sum_{\ell \in \mathbb{Z}_{m}} \int_{0}^{X}\left(y_{q}^{\ell}\left(X^{\prime}\right)-y_{q}^{\ell}(0)\right)\left(-\frac{d X^{\prime}}{X^{\prime}}\right) \cdot \psi_{\ell} ;
$$

this takes values in $H^{*}\left(\mathcal{B} \boldsymbol{\mu}_{m} ; \mathbb{C}\right)$.

(2) (Annulus invariants) The form

$$
\left(\rho_{q}^{\ell_{1}, \ell_{2}}\right)^{*} \omega_{0,2}-\frac{d X_{1} d X_{2}}{\left(X_{1}-X_{2}\right)^{2}}
$$

is holomorphic on $D_{\delta} \times D_{\delta}$. Define the $B$-model annulus potential by

$$
\check{F}_{0,2}\left(q ; X_{1}, X_{2}\right):=\sum_{\ell_{1}, \ell_{2} \in \mathbb{Z}_{m}} \int_{0}^{X_{1}} \int_{0}^{X_{2}}\left(\left(\rho_{q}^{\ell_{1}, \ell_{2}}\right)^{*} \omega_{0,2}-\frac{d X_{1}^{\prime} d X_{2}^{\prime}}{\left(X_{1}^{\prime}-X_{2}^{\prime}\right)^{2}}\right) \cdot \psi_{\ell_{1}} \otimes \psi_{\ell_{2}} ;
$$

this takes values in $H^{*}\left(\mathcal{B} \boldsymbol{\mu}_{m} ; \mathbb{C}\right)^{\otimes 2}$.

(3) For $2 g-2+n>0$, the form $\left(\rho_{\tau}^{\ell_{1}, \ldots, \ell_{n}}\right)^{*} \omega_{g, n}$ is holomorphic on $\left(D_{\delta}\right)^{n}$. Define

$$
\check{F}_{g, n}\left(q ; X_{1}, \ldots, X_{n}\right):=\sum_{\ell_{1}, \ldots, \ell_{n} \in \mathbb{Z}_{m}} \int_{0}^{X_{1}} \cdots \int_{0}^{X_{n}}\left(\rho_{q}^{\ell_{1}, \ldots, \ell_{n}}\right)^{*} \omega_{g, n} \cdot \psi_{\ell_{1}} \otimes \cdots \otimes \psi_{\ell_{n}} ;
$$


this takes values in $H^{*}\left(\mathcal{B} \boldsymbol{\mu}_{m} ; \mathbb{C}\right)^{\otimes n}$.

For $g \in \mathbb{Z}_{\geqslant 0}$ and $n \in \mathbb{Z}_{>0}$, the function $\check{F}_{g, n}\left(q ; X_{1}, \ldots, X_{n}\right)$ is holomorphic on $B_{\epsilon} \times\left(D_{\delta}\right)^{n}$ when $\epsilon, \delta>0$ are sufficiently small. By construction, the power series expansion of $\check{F}_{g, n}\left(q ; X_{1}, \ldots, X_{n}\right)$ involves only positive powers of $X_{i}$.

For $\beta \in G^{*}$, let $\xi_{\beta, 0}^{\ell-}$ be defined as in Section 7.3. Then $\xi_{\beta, 0}^{\ell-}(X)=\int_{0}^{X}\left(\rho_{q}^{\ell}\right)^{*} \theta_{0}^{\beta}$.

\subsection{Special geometry and B-model graph sum}

By the special geometry property of the topological recursion ([EO15, Theorem 4.4], proved in $[\mathrm{EO} 07])$,

$$
\nabla_{\partial / \partial \tau_{a}} \omega_{g, n}\left(z_{1}, \ldots, z_{n}\right)=\int_{z_{n+1} \in B_{a}} \omega_{g, n+1}\left(z_{1}, \ldots, z_{n+1}\right)
$$

where $B_{a}$ is defined as in Section 6.4. At $q=0$,

$$
\begin{aligned}
\int_{z_{n+1} \in B_{a}} \theta_{k}^{\alpha}\left(z_{n+1}\right) & =\left[u^{-k}\right] \int_{z_{n+1} \in B_{a}}\left(-\frac{\sqrt{u} e^{u a_{\alpha}}}{\sqrt{\pi}}\right) \int_{z^{\prime} \in \gamma_{\alpha}} B\left(z_{n+1}, z^{\prime}\right) e^{-u x\left(z^{\prime}\right)} \\
& =\left[u^{-k}\right]\left(-\frac{\sqrt{u} e^{u a_{\alpha}}}{\sqrt{\pi}}\right) \int_{z^{\prime} \in \gamma_{\alpha}}\left(\nabla_{\partial / \partial \tau_{a}} \Phi\right)\left(z^{\prime}\right) e^{-u x\left(z^{\prime}\right)} \\
& =\left[u^{-k}\right] \frac{1}{|G|} \sqrt{\frac{-2}{w_{1} w_{2} w_{3}}} \prod_{i=1}^{3} w_{i}^{c_{i}\left(h_{a}\right)} \sum_{\beta \in G^{*}} f_{\beta}^{\alpha}(u) \chi_{\beta}\left(h_{a}\right) .
\end{aligned}
$$

Define the B-model primary leaf to be

$$
\left(\check{h}^{\boldsymbol{\tau}}\right)_{k}^{\alpha}=\left[u^{-k}\right]\left(\frac{1}{|G|} \sqrt{\frac{-2}{w_{1} w_{2} w_{3}}} \sum_{\beta \in G^{*}} \sum_{a=1}^{p} \prod_{i=1}^{3} w_{i}^{c_{i}\left(h_{a}\right)} f_{\beta}^{\alpha}(u, 0) \chi_{\beta}\left(h_{a}\right) \tau_{a}\right) .
$$

For any $\alpha \in G^{*}, k \in \mathbb{Z}_{\geqslant 0}$, and $\ell \in \mathbb{Z}_{m}$, the form $\left(\rho_{0}^{\ell}\right)^{*} \theta_{k}^{\alpha}$ is a holomorphic 1 -form on $D_{\delta}$ for small enough $\delta>0$. To each open leaf $l_{j}$ of a labeled graph $\vec{\Gamma} \in \Gamma_{n}(\mathcal{X})$, we assign

$$
\left(\mathcal{L}^{\xi}\right)_{k}^{\alpha}\left(l_{j}\right)=\frac{1}{\sqrt{-2}} \sum_{\ell \in \mathbb{Z}_{m}}\left(\int_{0}^{X_{j}}\left(\rho_{0}^{\ell}\right)^{*} \theta_{k}^{\alpha}\right) \psi_{\ell}
$$

The Taylor series expansion of $\left(\rho_{\boldsymbol{\tau}}^{\ell_{1}, \ldots, \ell_{n}}\right)^{*} \omega_{g, n}$ at $\boldsymbol{\tau}=0$ is

$$
\left(\rho_{\boldsymbol{\tau}}^{\ell_{1}, \ldots, \ell_{n}}\right)^{*} \omega_{g, n}=\sum_{l_{1}, \ldots, l_{p} \in \mathbb{Z}_{\geqslant 0}}\left(\rho_{0}^{\ell_{1}, \ldots, \ell_{n}}\right)^{*}\left(\left.\left(\nabla_{\partial / \partial \tau_{1}}\right)^{l_{1}} \cdots\left(\nabla_{\partial / \partial \tau_{p}}\right)^{l_{p}} \omega_{g, n}\right|_{\boldsymbol{\tau}=0}\right) \cdot \frac{\tau_{1}^{l_{1}}}{l_{1} !} \cdots \frac{\tau_{p}^{l_{p}}}{l_{p} !} .
$$

Recall that $\boldsymbol{\Gamma}_{g, n}(\mathcal{X})$ is the set of stable genus $g$ graphs with $n$ open leaves and any number of primary leaves. Given $\vec{\Gamma}=(\Gamma, g, \alpha, k) \in \boldsymbol{\Gamma}_{g, n}(\mathcal{X})$ with open leaves $l_{1}, \ldots, l_{n}$, we define its B-model weight to be

$$
\begin{aligned}
w_{B}(\vec{\Gamma})= & \left.(-1)^{g(\vec{\Gamma})-1} \prod_{v \in V(\Gamma)}\left(\frac{\left.h_{1}^{\alpha}\right|_{\boldsymbol{\tau}=0}}{\sqrt{-2}}\right)^{2-2 g-\operatorname{val}(v)}\left\langle\prod_{h \in H(v)} \tau_{k(h)}\right\rangle_{g(v)} \prod_{e \in E(\Gamma)} \check{B}_{k(e), l(e)}^{\alpha\left(v_{1}(e)\right), \alpha\left(v_{2}(e)\right)}(e)\right|_{\boldsymbol{\tau}=0} \\
& \left.\cdot \prod_{l \in L^{o}(\Gamma)} \frac{1}{\sqrt{-2}}\left(\check{h}^{\boldsymbol{\tau}}\right)_{k(l)}^{\alpha(l)} \cdot \prod_{j=1}^{n}\left(\mathcal{L}^{\xi}\right)_{k}^{\alpha}\left(l_{j}\right) \prod_{l \in L^{1}(\Gamma)}\left(-\frac{1}{\sqrt{-2}}\right) \check{h}_{k(l)}^{\alpha(l)}(l)\right|_{\boldsymbol{\tau}=0} .
\end{aligned}
$$

We have the following B-model graph sum formulae. 


\section{All-GenUs OPEN-CLOSED MIRROR SYMMETRY}

Theorem 7.15 (B-model graph sum). (i) (Disk invariants)

$$
\check{F}_{0,1}(\boldsymbol{\tau} ; X)=\check{F}_{0,1}(0 ; X)+\sum_{a=1}^{p} \tau_{a} \frac{\partial \check{F}_{0,1}}{\partial \tau_{a}}(0 ; X)+\sum_{\vec{\Gamma} \in \boldsymbol{\Gamma}_{0,1}(\mathcal{X})} \frac{w_{B}(\vec{\Gamma})}{|\operatorname{Aut}(\vec{\Gamma})|} .
$$

(ii) (Annulus invariants)

$$
\check{F}_{0,2}\left(\boldsymbol{\tau} ; X_{1}, X_{2}\right)=\check{F}_{0,2}\left(0 ; X_{1}, X_{2}\right)+\sum_{\vec{\Gamma} \in \boldsymbol{\Gamma}_{0,2}(\mathcal{X})} \frac{w_{B}(\vec{\Gamma})}{|\operatorname{Aut}(\vec{\Gamma})|} .
$$

(iii) For $2 g-2+n>0$,

$$
\check{F}_{g, n}\left(\boldsymbol{\tau} ; X_{1}, \ldots, X_{n}\right)=\sum_{\vec{\Gamma} \in \boldsymbol{\Gamma}_{g, n}(\mathcal{X})} \frac{w_{B}(\vec{\Gamma})}{|\operatorname{Aut}(\vec{\Gamma})|}
$$

\subsection{All-genus open-closed mirror symmetry}

Proposition 7.16. We have

$$
\check{F}_{0,1}(0 ; X)+\sum_{a=1}^{p} \tau_{a} \frac{\partial \check{F}_{0,1}}{\partial \tau_{a}}(0 ; X)=\Phi_{1,-2}(X)+\sum_{a=1}^{p} \tau_{a} \Phi_{h_{a},-1}(X) .
$$

Proof. From the definition,

$$
-X \frac{d}{d X} \check{F}_{0,1}(0 ; X)=\sum_{\ell \in \mathbb{Z}_{m}}\left(y_{0}^{\ell}(X)-y_{0}^{\ell}(0)\right) \psi_{\ell} .
$$

By Proposition 6.4, equations (6.13) and (7.2), and Proposition 7.11,

$$
\begin{aligned}
& \left(X \frac{d}{d X}\right)^{2} \check{F}_{0,1}(0 ; X)=\sum_{\beta \in G^{*}} \frac{1}{|G| \sqrt{-2 w_{1} w_{2} w_{3}}} \lim _{q \rightarrow 0} \xi_{0}^{\beta}(X)=\left.\sum_{\beta \in G^{*}} \frac{1}{|G|^{2} w_{1} w_{2} w_{3}} \widetilde{\xi}_{0}^{\beta}(X)\right|_{\mathrm{v}=1}, \\
& \left(X \frac{d}{d X}\right) \frac{\partial \check{F}_{0,1}}{\partial \tau_{a}}(0 ; X)=\frac{1}{|G| \sqrt{-2 w_{1} w_{2} w_{3}}} \sum_{\beta \in G^{*}} \chi_{\beta}\left(h_{a}\right) \prod_{i=1}^{3} w_{i}^{c_{i}\left(h_{a}\right)} \lim _{q \rightarrow 0} \xi_{0}^{\beta}(X) \\
& =\left.\frac{1}{|G|^{2} w_{1} w_{2} w_{3}} \sum_{\beta \in G^{*}} \chi_{\beta}\left(h_{a}\right) \prod_{i=1}^{3} w_{i}^{c_{i}\left(h_{a}\right)} \widetilde{\xi}_{0}^{\beta}(X)\right|_{\mathrm{v}=1} .
\end{aligned}
$$

The functions $\check{F}_{0,1}(0 ; X)$ and $\left(\partial \check{F}_{0,1} / \partial \tau_{a}\right)(0 ; X)$ are power series in $X$ with no constant term, so

$$
\begin{gathered}
\check{F}_{0,1}(0 ; X)=\left.\frac{1}{|G|^{2} w_{1} w_{2} w_{3}} \widetilde{\xi}_{-2}^{\beta}(X)\right|_{\mathrm{v}=1}, \\
\frac{\partial \check{F}_{0,1}}{\partial \tau_{a}}(0 ; X)=\left.\frac{1}{|G|^{2} w_{1} w_{2} w_{3}} \sum_{\beta \in G^{*}} \chi_{\beta}\left(h_{a}\right) \prod_{i=1}^{3} w_{i}^{c_{i}\left(h_{a}\right)} \widetilde{\xi}_{-1}^{\beta}(X)\right|_{\mathrm{v}=1} .
\end{gathered}
$$

The lemma follows from (7.15), (7.16), and (5.11).

Proposition 7.17. We have $\check{F}_{0,2}\left(0 ; X_{1}, X_{2}\right)=-F_{0,2}^{\mathcal{X},(\mathcal{L}, f)}\left(0 ; X_{1}, X_{2}\right)$.

Proof. Let $C=C\left(z_{1}, z_{2}\right)$ be defined by (7.11). Then

$$
\left(X_{1} \frac{\partial}{\partial X_{1}}+X_{2} \frac{\partial}{\partial X_{2}}\right) \check{F}_{0,2}\left(0 ; X_{1}, X_{2}\right)=\sum_{\ell_{1}, \ell_{2} \in \mathbb{Z}_{m}} \int_{0}^{X_{1}} \int_{0}^{X_{2}}\left(\rho_{0}^{\ell_{1}, \ell_{2}}\right)^{*} C \psi_{\ell_{1}} \otimes \psi_{\ell_{2}}
$$


B. FAnG, C.-C. M. LiU And Z. Zong

$$
\begin{aligned}
& =\frac{1}{2} \sum_{\ell_{1}, \ell_{2} \in \mathbb{Z}_{m}} \sum_{\gamma \in G^{*}} \int_{0}^{X_{1}}\left(\rho_{0}^{\ell_{1}}\right)^{*} \theta_{0}^{\gamma} \int_{0}^{X_{2}}\left(\rho_{0}^{\ell_{2}}\right)^{*} \theta_{0}^{\gamma} \psi_{\ell_{1}} \otimes \psi_{\ell_{2}} \\
& =\lim _{q \rightarrow 0} \frac{1}{2} \sum_{\gamma \in G^{*}} \xi_{0}^{\gamma}\left(X_{1}\right) \xi_{0}^{\gamma}\left(X_{2}\right) \\
& =-\left.\frac{1}{|G|^{2} w_{1} w_{2} w_{3}}\left(\sum_{\gamma \in G^{*}} \widetilde{\xi}_{0}^{\gamma}\left(X_{1}\right) \widetilde{\xi}_{0}^{\gamma}\left(X_{2}\right)\right)\right|_{v=1} \\
& =-\left(X_{1} \frac{\partial}{\partial X_{1}}+X_{2} \frac{\partial}{\partial X_{2}}\right) F_{0,2}^{\mathcal{X},(\mathcal{L}, f)}\left(0 ; X_{1}, X_{2}\right),
\end{aligned}
$$

where the second equality follows from Lemma 7.14, the fourth equality follows from Proposition 7.11, and the last equality follows from (5.5). Both $W_{0,2}\left(0 ; X_{1}, X_{2}\right)$ and $F_{0,2}^{\mathcal{X},(\mathcal{L}, f)}\left(0 ; X_{1}, X_{2}\right)$ are $H_{\mathrm{CR}}^{*}\left(\mathcal{B} \boldsymbol{\mu}_{m} ; \mathbb{C}\right)^{\otimes 2}$-valued power series in $X_{1}$ and $X_{2}$ which vanish at $\left(X_{1}, X_{2}\right)=(0,0)$, so

$$
\check{F}_{0,2}\left(0 ; X_{1}, X_{2}\right)=-F_{0,2}^{\mathcal{X},(\mathcal{L}, f)}\left(0 ; X_{1}, X_{2}\right) \text {. }
$$

Proposition 7.18. For any $\vec{\Gamma} \in \boldsymbol{\Gamma}_{g, n}(\mathcal{X})$, we have

$$
w_{B}(\vec{\Gamma})=(-1)^{g(\vec{\Gamma})-1+n} w_{A}(\vec{\Gamma}),
$$

where $w_{A}(\vec{\Gamma})$ is defined in Section 5.3 and $w_{B}(\vec{\Gamma})$ is defined in Section 7.6.

Proof. We fix $\vec{\Gamma} \in \boldsymbol{\Gamma}_{g, n}(\mathcal{X})$. From (5.7) and Theorem 7.8, for any $\alpha, \beta \in G^{*}$,

$$
R(-z)_{\alpha}^{\beta}=f_{\beta}^{\alpha}\left(\frac{1}{z}, 0\right)
$$

Here we have used the fact that $B_{m}(1-x)=(-1)^{m} B_{m}(x), B_{m}=0$ when $m$ is odd and $c_{i}(h)+c_{i}\left(h^{-1}\right)=1-\delta_{c_{i}(h), 0}$.

(1) (Vertex) By equation (7.2), we have

$$
\frac{\lim _{\boldsymbol{\tau} \rightarrow 0} h_{1}^{\alpha}}{\sqrt{-2}}=\frac{1}{|G| \sqrt{w_{1} w_{2} w_{3}}} .
$$

(2) (Edge) By equation (7.10) (that is, [Eyn11, equation (B.9)]), we have

$$
\begin{aligned}
\left.\check{B}_{k, l}^{\alpha, \beta}\right|_{\boldsymbol{\tau}=0} & =\left[u^{-k} v^{-l}\right]\left(\frac{u v}{u+v}\left(\delta_{\alpha, \beta}-\sum_{\gamma \in G^{*}} f_{\gamma}^{\alpha}(u, 0) f_{\gamma}^{\beta}(v, 0)\right)\right) \\
& =\left[z^{k} w^{l}\right]\left(\frac{1}{z+w}\left(\delta_{\alpha, \beta}-\sum_{\gamma \in G^{*}} f_{\gamma}^{\alpha}\left(\frac{1}{z}, 0\right) f_{\gamma}^{\beta}\left(\frac{1}{w}, 0\right)\right)\right) .
\end{aligned}
$$

By definition,

$$
\mathcal{E}_{k, l}^{\alpha, \beta}=\left[z^{k} w^{l}\right]\left(\frac{1}{z+w}\left(\delta_{\alpha, \beta}-\sum_{\gamma \in G^{*}} R(-z)_{\alpha}^{\gamma} R(-w)_{\beta}^{\gamma}\right)\right) .
$$

It follows from (7.17) that

$$
\left.\check{B}_{k, l}^{\alpha, \beta}\right|_{\boldsymbol{\tau}=0}=\mathcal{E}_{k, l}^{\alpha, \beta}
$$

(3) (Primary leaf) By equation (7.12),

$$
\frac{1}{\sqrt{-2}}\left(\check{h}^{\boldsymbol{\tau}}\right)_{k}^{\alpha}=\left[u^{-k}\right]\left(\frac{1}{|G| \sqrt{w_{1} w_{2} w_{3}}} \sum_{\beta \in G^{*}} \sum_{a=1}^{p} \prod_{i=1}^{3} w_{i}^{c_{i}\left(h_{a}\right)} f_{\beta}^{\alpha}(u) \chi_{\beta}\left(h_{a}\right) \tau_{a}\right) .
$$




\section{All-Genus OPEN-CLOSED MIRROR SYMMETRY}

By equation (5.8),

$$
\left(\mathcal{L}^{\tau}\right)_{k}^{\alpha}=\left[z^{k}\right]\left(\frac{1}{|G| \sqrt{w_{1} w_{2} w_{3}}} \sum_{\beta \in G^{*}} \sum_{a=1}^{p} \prod_{i=1}^{3} w_{i}^{c_{i}\left(h_{a}\right)} R(-z)_{\alpha}^{\beta} \chi_{\beta}\left(h_{a}\right) \tau_{a}\right) .
$$

It follows from (7.17) that

$$
\frac{1}{\sqrt{-2}}\left(\check{h}^{\boldsymbol{\tau}}\right)_{k}^{\alpha}=\left(\mathcal{L}^{\boldsymbol{\tau}}\right)_{k}^{\alpha}
$$

(4) (Open leaf) Given $\beta \in G^{*}$ and $k \in \mathbb{Z}_{\geqslant 0}$, define

$$
\hat{\xi}_{k}^{\beta}(X):=\sum_{\ell=0}^{m-1} \int_{0}^{X}\left(\rho_{0}^{\ell}\right)^{*}\left(d \hat{\xi}_{\beta, k}\right) \psi_{\ell},
$$

which is an $H_{\mathrm{CR}}^{*}\left(\mathcal{B} \boldsymbol{\mu}_{m} ; \mathbb{C}\right)$-valued holomorphic function on $D_{\delta}$. Then $\hat{\xi}_{k}^{\beta}(X)=(X d / d X)^{k} \hat{\xi}_{0}^{\beta}(X)$ and $\hat{\xi}_{0}^{\beta}(X)=\lim _{q \rightarrow 0} \xi_{0}^{\beta}$, where $\xi_{0}^{\beta}$ is defined by equation (7.8). By Proposition 7.11 and the definitions of $\hat{\xi}_{k}^{\beta}(X)$ and $\widetilde{\xi}_{k}^{\beta}(X)$,

$$
\hat{\xi}_{k}^{\beta}(X)=-\frac{1}{|G|} \sqrt{\frac{-2}{w_{1} w_{2} w_{3}}} \widetilde{\xi}_{k}^{\beta}(X) .
$$

By (7.13), (7.21), (7.22), and Lemma 6.7,

$$
\left(\mathcal{L}^{\xi}\right)_{k}^{\alpha}\left(l_{j}\right)=\frac{-1}{|G| \sqrt{w_{1} w_{2} w_{3}}}\left(\widetilde{\xi}_{k}^{\alpha}-\sum_{i=0}^{k-1} \sum_{\beta \in G^{*}}\left(\left.\check{B}_{k-1-i, 0}^{\alpha, \beta}\right|_{\tau=0}\right) \widetilde{\xi}_{i}^{\beta}\left(X_{j}\right)\right) .
$$

By item (2) above, for $k \in \mathbb{Z}_{\geqslant 0}$,

$$
\left.\check{B}_{k, 0}^{\alpha, \beta}\right|_{\tau=0}=\left[z^{k} w^{0}\right]\left(\frac{1}{z+w}\left(\delta_{\alpha, \beta}-\sum_{\gamma \in G^{*}} R(-z)_{\alpha}^{\gamma} R(-w)_{\alpha}^{\gamma}\right)\right)=\left[z^{k+1}\right]\left(-R(-z)_{\alpha}^{\beta}\right) .
$$

We also have $\left[z^{0}\right]\left(R(-z)_{\alpha}^{\beta}\right)=\delta_{\alpha, \beta}$. Therefore,

$$
\left(\mathcal{L}^{\xi}\right)_{k}^{\alpha}\left(l_{j}\right)=\left[z^{k}\right]\left(\frac{-1}{|G| \sqrt{w_{1} w_{2} w_{3}}} \sum_{\beta \in G^{*}} R(-z)_{\alpha}^{\beta} \widetilde{\xi}^{\beta}\left(z, X_{j}\right)\right) .
$$

Comparing the above expression with the definition of the A-model open leaf $\left(\mathcal{L}^{\widetilde{\xi}}\right)_{k}^{\alpha}\left(l_{j}\right)$, we conclude that

(5) (Dilaton leaf)

$$
\left(\mathcal{L}^{\xi}\right)_{k}^{\alpha}\left(l_{j}\right)=-\left(\mathcal{L}^{\widetilde{\xi}}\right)_{k}^{\alpha}\left(l_{j}\right)
$$

$$
\left.\left(-\frac{1}{\sqrt{-2}}\right) \check{h}_{k}^{\alpha}\right|_{\tau=0}=\left.\left[u^{1-k}\right]\left(-\frac{1}{\sqrt{-2}}\right) \check{h}^{\alpha}(u)\right|_{\tau=0}=\left[u^{1-k}\right] \frac{-1}{|G| \sqrt{w_{1} w_{2} w_{3}}} \sum_{\beta \in G^{*}} f_{\beta}^{\alpha}(u, 0),
$$

where the second identity follows from Corollary 7.9. By definition,

$$
\left(\mathcal{L}^{1}\right)_{k}^{\alpha}=\left[z^{k-1}\right] \frac{-1}{|G| \sqrt{w_{1} w_{2} w_{3}}} \sum_{\beta \in G^{*}} R_{\alpha}^{\beta}(-z) .
$$

It follows from (7.17) that

$$
\left.\left(-\frac{1}{\sqrt{-2}}\right) \check{h}_{k}^{\alpha}\right|_{\tau=0}=\left(\mathcal{L}^{1}\right)_{k}^{\alpha}
$$




\section{B. FAnG, C.-C. M. LiU And Z. Zong}

By (5.9), (7.14), (7.18), (7.19), (7.20), (7.23), and (7.24),

$$
w_{B}(\vec{\Gamma})=(-1)^{g(\vec{\Gamma})-1+n} w_{A}(\vec{\Gamma}) .
$$

Combining Theorem 5.5 (A-model graph sum), Theorem 7.15 (B-model graph sum), and Propositions 7.16, 7.17, and 7.18, we obtain the final result.

Theorem 7.19 (All-genus open-closed mirror symmetry). We have

$$
\check{F}_{g, n}\left(\boldsymbol{\tau} ; X_{1}, \ldots, X_{n}\right)=(-1)^{g-1+n} F_{g, n}^{\mathcal{X},(\mathcal{L}, f)}\left(\boldsymbol{\tau} ; X_{1}, \ldots, X_{n}\right) .
$$

As a consequence, the $H^{*}\left(\mathcal{B} \boldsymbol{\mu}_{m}\right)^{\otimes n}$-valued formal power series $F_{g, n}^{\mathcal{X},(\mathcal{L}, f)}\left(\boldsymbol{\tau} ; X_{1}, \ldots, X_{n}\right)$ converges in an open neighborhood of the origin in $\mathbb{C}^{p} \times \mathbb{C}^{n}$.

\section{ACKNOWLEDGEMENTS}

We thank Vincent Bouchard, Igor Dolgachev, Bertrand Eynard, Robert Friedman, Nicolas Orantin, Dustin Ross and Jie Zhou for helpful comments and conversations.

\section{REFERENCES}

AF16 D. Abramovich and B. Fantechi, Orbifold techniques in degeneration formulas, Ann. Sc. Norm. Super. Pisa Cl. Sci. (5) 16 (2016), no. 2, 519-579; doi:10.2422/2036-2145.201408_006.

AGV02 D. Abramovich, T. Graber, and A. Vistoli, Algebraic orbifold quantum products, Orbifolds in Mathematics and Physics (Madison, WI, 2001), Contemp. Math., vol. 310 (Amer. Math. Soc., Providence, RI, 2002), 1-24; doi:10.1090/conm/310/05397.

AGV08_ Gromov-Witten theory of Deligne-Mumford stacks, Amer. J. Math. 130 (2008), no. 5, 1337-1398; doi:10.1353/ajm.0.0017.

AKMV05 M. Aganagic, A. Klemm, M. Mariño, and C. Vafa, The topological vertex, Comm. Math. Phys. 254 (2005), no. 2, 425-478; doi:10.1007/s00220-004-1162-z.

AKV02 M. Aganagic, A. Klemm, and C. Vafa, Disk instantons, mirror symmetry and the duality web, Z. Naturforsch. A 57 (2002), no. 1-2, 1-28; doi:10.1515/zna-2002-9-1001.

AV00 M. Aganagic and C. Vafa, Mirror symmetry, D-branes and counting holomorphic discs, 2000, arXiv:hep-th/0012041.

BC94 V.V. Batyrev and D. A. Cox, On the Hodge structure of projective hypersurfaces in toric varieties, Duke Math. J. 75 (1994), no. 2, 293-338; doi:10.1215/S0012-7094-94-07509-1.

BCMS13 V. Bouchard, A. Catuneanu, O. Marchal, and P. Sułkowski, The remodeling conjecture and the Faber-Pandharipande formula, Lett. Math. Phys. 103 (2013), no. 1, 59-77; doi:10.1007/ s11005-012-0588-z.

BCOV93 M. Bershadsky, S. Cecotti, H. Ooguri, and C. Vafa, Holomorphic anomalies in topological field theories, Nuclear Phys. B 405 (1993), no. 2-3, 279-304; doi:10.1016/0550-3213(93) 90548-4.

BCY12 J. Bryan, C. Cadman, and B. Young, The orbifold topological vertex, Adv. Math. 229 (2012), no. 1, 531-595; doi:10.1016/j.aim.2011.09.008.

BHLM14 V. Bouchard, D. Hernández Serrano, X. Liu, and M. Mulase, Mirror symmetry for orbifold Hurwitz numbers, J. Differential Geom. 98 (2014), no. 3, 375-423; doi:10.4310/jdg/1406552276.

BKMP09 V. Bouchard, A. Klemm, M. Mariño, and S. Pasquetti, Remodeling the B-model, Comm. Math. Phys. 287 (2009), no. 1, 117-178; doi:10.1007/s00220-008-0620-4.

BKMP10 , Topological open strings on orbifolds, Comm. Math. Phys. 296 (2010), no. 3, 589-623; doi:10.1007/s00220-010-1020-0. 


\section{ALL-GENUS OPEN-CLOSED MIRROR SYMMETRY}

BR87 M. Bershadsky and A. Radul, Conformal field theories with additional $Z_{N}$ symmetry, Internat. J. Modern Phys. A 2 (1987), no. 1, 165-178; doi:10.1142/S0217751X87000053.

BS12 V. Bouchard and P. Sułkowski, Topological recursion and mirror curves, Adv. Theor. Math. Phys. 16 (2012), no. 5, 1443-1483; doi:10.4310/atmp.2012.v16.n5.a3.

CCIT15 T. Coates, A. Corti, H. Iritani, and H.-H. Tseng, A mirror theorem for toric stacks, Compos. Math. 151 (2015), no. 10, 1878-1912; doi:10.1112/S0010437X15007356.

CCK15 D. Cheong, I. Ciocan-Fontanine, and B. Kim, Orbifold quasimap theory, Math. Ann. 363 (2015), no. 3-4, 777-816; doi:10.1007/s00208-015-1186-z.

Che18 L. Chen, Bouchard-Klemm-Mariño-Pasquetti conjecture for $\mathbb{C}^{3}$, in Topological Recursion and its Influence in Analysis, Geometry, and Topology, Proc. Sympos. Pure Math., vol. 100 (Amer. Math. Soc., Providence, RI, 2018), 83-102.

CKYZ99 T.-M. Chiang, A. Klemm, S.-T. Yau, and E. Zaslow, Local mirror symmetry: calculations and interpretations, Adv. Theor. Math. Phys. 3 (1999), no. 3, 495-565; doi:10.4310/ATMP.1999. v3.n3.a3.

CLSZ11 B. Chen, A.-M. Li, S. Sun, and G. Zhao, Relative orbifold Gromov-Witten theory and degeneration formula, 2011, arXiv:1110.6803.

CR02 W. Chen and Y. Ruan, Orbifold Gromov-Witten theory, Orbifolds in Mathematics and Physics (Madison, WI, 2001), Contemp. Math., vol. 310 (Amer. Math. Soc., Providence, RI, 2002), 25-85; doi:10.1090/conm/310/05398.

CR04 A A new cohomology theory of orbifold, Comm. Math. Phys. 248 (2004), no. 1, 1-31; doi:10.1007/s00220-004-1089-4.

CV92 S. Cecotti and C. Vafa, Massive orbifolds, Modern Phys. Lett. A 7 (1992), no. 19, 1715-1723; doi:10.1142/S0217732392001415.

DF05 D.-E. Diaconescu and B. Florea, Localization and gluing of topological amplitudes, Comm. Math. Phys. 257 (2005), no. 1, 119-149; doi:10.1007/s00220-005-1323-8.

DFMS87 L. Dixon, D. Friedan, E. Martinec, and S. Shenker, The conformal field theory of orbifolds, Nuclear Phys. B 282 (1987), no. 1, 13-73; doi:10.1016/0550-3213(87)90676-6.

DHVW85 L. Dixon, J. Harvey, C. Vafa, and E. Witten, Strings on orbifolds, Nuclear Phys. B 261 (1985), no. 4, 678-686; doi:10.1016/0550-3213(85)90593-0.

DHVW86_ Strings on orbifolds. II, Nuclear Phys. B 274 (1986), no. 2, 285-314; doi:10.1016/ 0550-3213 (86) 90287-7.

DOSS14 P. Dunin-Barkowski, N. Orantin, S. Shadrin, and L. Spitz, Identification of the Givental formula with the spectral curve topological recursion procedure, Comm. Math. Phys. 328 (2014), no. 2, 669-700; doi:10.1007/s00220-014-1887-2.

EO07 B. Eynard and N. Orantin, Invariants of algebraic curves and topological expansion, Commun. Number Theory Phys. 1 (2007), no. 2, 347-452; doi:10.4310/CNTP.2007.v1.n2.a4.

EO15_ Computation of open Gromov-Witten invariants for toric Calabi-Yau 3-folds by topological recursion, a proof of the BKMP conjecture, Comm. Math. Phys. 337 (2015), no. 2, 483-567; doi:10.1007/s00220-015-2361-5.

Eyn11 B. Eynard, Intersection numbers of spectral curves, 2011, arXiv:1104.0176.

Eyn14 Invariants of spectral curves and intersection theory of moduli spaces of complex curves, Commun. Number Theory Phys. 8 (2014), no. 3, 541-588; doi:10.4310/CNTP . 2014. v8.n3.a4.

Fay73 J.D. Fay, Theta functions on Riemann surfaces, Lecture Notes in Math., vol. 352 (SpringerVerlag, Berlin - New York, 1973); doi:10.1007/BFb0060090.

FLT12 B. Fang, C.-C.M. Liu, and H.-H. Tseng, Open-closed Gromov-Witten invariants of 3dimensional Calabi-Yau smooth toric DM stacks, 2012, arXiv:1212.6073. 


\section{B. FAnG, C.-C. M. LiU And Z. ZonG}

FLZ16 B. Fang, C.-C.M. Liu, and Z. Zong, Equivariant Gromov-Witten theory of affine smooth toric Deligne-Mumford stacks, Int. Math. Res. Not. 2016 (2016), no. 7, 2127-2144; doi: 10.1093/imrn/rnv201.

FLZ19 - On the remodeling conjecture for toric Calabi-Yau 3-orbifolds, J. Amer. Math. Soc., published online on 1 November 2019, doi:10.1090/jams/934, to appear in print.

Giv96 A. Givental, Equivariant Gromov-Witten invariants, Int. Math. Res. Not. 1996 (1996), no. 13, 613-663; doi:10.1155/S1073792896000414.

Giv98_ A mirror theorem for toric complete intersections, Topological Field Theory, Primitive Forms and Related Topics (Kyoto, 1996), Progr. Math., vol. 160 (Birkhäuser Boston, Boston, MA, 1998), 141-175; doi:10.1007/978-1-4612-0705-4_5.

GP99 T. Graber and R. Pandharipande, Localization of virtual classes, Invent. Math. 135 (1999), no. 2, 487-518; doi:10.1007/s002220050293.

HIV00 K. Hori, A. Iqbal, and C. Vafa, D-branes and mirror symmetry, 2000, arXiv: hep-th/0005247.

HKQ09 M.X. Huang, A. Klemm, and S. Quackenbush, Topological string theory on compact CalabiYau: modularity and boundary conditions, in Homological Mirror Symmetry, Lecture Notes in Phys., vol. 757 (Springer, Berlin, 2009), 45-102; doi:10.1007/978-3-540-68030-7_3.

HL82 R. Harvey and H.B. Lawson, Jr., Calibrated geometries, Acta Math. 148 (1982), 47-157; doi:10.1007/BF02392726.

HV87 S. Hamidi and C. Vafa, Interactions on orbifolds, Nuclear Phys. B 279 (1987), no. 3-4, 465-513; doi:10.1016/0550-3213(87)90006-X.

Iri09 H. Iritani, An integral structure in quantum cohomology and mirror symmetry for toric orbifolds, Adv. Math. 222 (2009), no. 3, 1016-1079; doi:10.1016/j.aim.2009.05.016.

JK02 T. J. Jarvis and T. Kimura, Orbifold quantum cohomology of the classifying space of a finite group, Orbifolds in Mathematics and Physics (Madison, WI, 2001), Contemp. Math., vol. 310 (Amer. Math. Soc., Providence, RI, 2002), 123-134; doi:10.1090/conm/310/05401.

KL01 S. Katz and C.-C.M. Liu, Enumerative geometry of stable maps with Lagrangian boundary conditions and multiple covers of the disc, Adv. Theor. Math. Phys. 5 (2001), no. 1, 1-49; doi:10.4310/ATMP.2001.v5.n1.a1.

Kni87 V.G. Knizhnik, Analytic fields on Riemann surfaces. II, Comm. Math. Phys. 112 (1987), no. 4, 567-590; doi:10.1007/bf01225373.

KO10 I. Kostov and N. Orantin, CFT and topological recursion, J. High Energy Phys. 2010 (2010), no. 11, 056; doi:10.1007/JHEP11(2010) 056.

KP11 J. Kaczorowski and A. Perelli, A uniform version of Stirling's formula, Funct. Approx. Comment. Math. 45 (2011), no. part 1, 89-96; doi:10.7169/facm/1317045234.

Liu02 C.-C.M. Liu, Moduli of J-holomorphic curves with Lagrangian boundary conditions and open Gromov-Witten invariants for an $S^{1}$-equivariant pair, 2002, arXiv:math.SG/0211388.

LLLZ09 J. Li, C.-C. M. Liu, K. Liu, and J. Zhou, A mathematical theory of the topological vertex, Geom. Topol. 13 (2009), no. 1, 527-621; doi:10.2140/gt.2009.13.527.

LLY97 B. H. Lian, K. Liu, and S.-T. Yau, Mirror principle. I, Asian J. Math. 1 (1997), no. 4, 729-763; doi:10.4310/AJM.1997.v1.n4.a5.

LLY99a Mirror principle. II, Asian J. Math. 3 (1999), no. 1, 109-146; doi:10.4310/AJM. 1999.v3.n1.a6.

LLY99b - Mirror principle. III, Asian J. Math. 3 (1999), no. 4, 771-800; doi:10.4310/AJM. 1999.v3.n4.a4.

Mar08 M. Mariño, Open string amplitudes and large order behavior in topological string theory, J. High Energy Phys. 2008 (2008), no. 3, 060; doi:10.1088/1126-6708/2008/03/060.

MNOP06 D. Maulik, N. Nekrasov, A. Okounkov, and R. Pandharipande, Gromov-Witten theory and Donaldson-Thomas theory. I, Compos. Math. 142 (2006), no. 5, 1263-1285; doi:10.1112/ S0010437X06002302. 


\section{AlL-GENUS OPEN-CLOSED MIRROR SYMMETRY}

MOOP11 D. Maulik, A. Oblomkov, A. Okounkov, and R. Pandharipande, Gromov-Witten/DonaldsonThomas correspondence for toric 3-folds, Invent. Math. 186 (2011), no. 2, 435-479; doi: 10.1007/s00222-011-0322-y.

MP06 D. Maulik and R. Pandharipande, A topological view of Gromov-Witten theory, Topology 45 (2006), no. 5, 887-918; doi:10.1016/j.top.2006.06.002.

Roa90 S.-S. Roan, On Calabi-Yau orbifolds in weighted projective spaces, Internat. J. Math. 1 (1990), no. 2, 211-232; doi:10.1142/S0129167X90000137.

Ros14 D. Ross, Localization and gluing of orbifold amplitudes: the Gromov-Witten orbifold vertex, Trans. Amer. Math. Soc. 366 (2014), no. 3, 1587-1620; doi:10.1090/ S0002-9947-2013-05835-7.

Ros15_ On the Gromov-Witten/Donaldson-Thomas correspondence and Ruan's conjecture for Calabi-Yau 3-orbifolds, Comm. Math. Phys. 340 (2015), no. 2, 851-864; doi:10.1007/ s00220-015-2438-1.

RZ13 D. Ross and Z. Zong, The gerby Gopakumar-Mariño-Vafa formula, Geom. Topol. 17 (2013), no. 5, 2935-2976; doi:10.2140/gt.2013.17.2935.

RZ15_ Cyclic Hodge integrals and loop Schur functions, Adv. Math. 285 (2015), 1448-1486; doi:10.1016/j.aim.2015.08.023.

Tse10 H.-H. Tseng, Orbifold quantum Riemann-Roch, Lefschetz and Serre, Geom. Topol. 14 (2010), no. 1, 1-81; doi:10.2140/gt.2010.14.1.

YY04 S. Yamaguchi and S.-T. Yau, Topological string partition functions as polynomials, J. High Energy Phys. 2004 (2004), no. 7, 047, 20 pp.; doi:10.1088/1126-6708/2004/07/047.

Zas93 E. Zaslow, Topological orbifold models and quantum cohomology rings, Comm. Math. Phys. 156 (1993), no. 2, 301-331; doi:10.1007/bf02098485.

Zho09a J. Zhou, Local mirror symmetry for one-legged topological vertex, 2009, arXiv:0910.4320.

Zho09b_ Local mirror symmetry for the topological vertex, 2009, arXiv:0911.2343.

Zhu15 S. Zhu, On a proof of the Bouchard-Sulkowski conjecture, Math. Res. Lett. 22 (2015), no. 2, 633-643; doi:10.4310/MRL.2015.v22.n2.a14.

Zin09 A. Zinger, The reduced genus 1 Gromov-Witten invariants of Calabi-Yau hypersurfaces, J. Amer. Math. Soc. 22 (2009), no. 3, 691-737; doi:10.1090/S0894-0347-08-00625-5.

Zon15 Z. Zong, Generalized Mariño-Vafa formula and local Gromov-Witten theory of orbi-curves, J. Differential Geom. 100 (2015), no. 1, 161-190; doi:10.4310/jdg/1427202767.

Zon16 Equivariant Gromov-Witten theory of GKM orbifolds, 2016, arXiv:1604.07270.

Bohan Fang bohanfang@gmail.com

Beijing International Center for Mathematical Research, Peking University, 5 Yiheyuan Road, Beijing 100871, China

Chiu-Chu Melissa Liu ccliu@math.columbia.edu

Department of Mathematics, Columbia University, 2990 Broadway, New York, NY 10027, USA

Zhengyu Zong zyzong@math.tsinghua.edu.cn

Yau Mathematical Sciences Center, Tsinghua University, Jin Chun Yuan West Building, Haidian

District, Beijing 100084, China 